\title{
Two-step solvable SKT shears
}

\author{
Marco Freibert ${ }^{1} \cdot$ Andrew Swann ${ }^{2}$
}

Received: 25 November 2020 / Accepted: 30 March 2021 / Published online: 18 April 2021

(c) The Author(s) 2021

\begin{abstract}
We use the shear construction to construct and classify a wide range of two-step solvable Lie groups admitting a left-invariant SKT structure. We reduce this to a specification of SKT shear data on Abelian Lie algebras, and which then is studied more deeply in different cases. We obtain classifications and structure results for $\mathfrak{g}$ almost Abelian, for derived algebra $\mathfrak{g}^{\prime}$ of codimension 2 and not $J$-invariant, for $\mathfrak{g}^{\prime}$ totally real, and for $\mathfrak{g}^{\prime}$ of dimension at most 2 . This leads to a large part of the full classification for two-step solvable SKT algebras of dimension six.
\end{abstract}

Mathematics Subject Classification Primary 53C55; Secondary 22E25

\section{Contents}

1 Introduction . . . . . . . . . . . . . . . . . . . . . . . . . . 1704

2 Two-step solvable algebras . . . . . . . . . . . . . . . . . . . . . . . . . . . . . 1706

3 Data for two-step solvable SKT algebras . . . . . . . . . . . . . . . . . . . . . . . . 1707

4 Two-step SKT shears with totally real complement . . . . . . . . . . . . . . . . . . . . . . . . 1712

4.1 Almost Abelian algebras . . . . . . . . . . . . . . . . . . . . . . . . . . . . . . . 1714

4.2 Commutator ideal not complex and of codimension two . . . . . . . . . . . . . . 1715

5 Commutator ideal totally real . . . . . . . . . . . . . . . . . . . . . . . . 1720

6 Commutator ideal complex of real-dimension two . . . . . . . . . . . . . . . . . . . . 1726

7 Six-dimensional two-step solvable SKT Lie algebras . . . . . . . . . . . . . . . . . . . . . . . . 1729

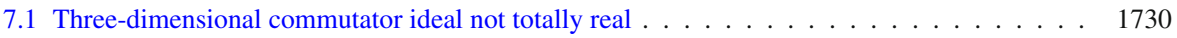

7.2 Four-dimensional complex commutator ideal . . . . . . . . . . . . . . . . . . . . 1737

Appendix A: Notation for particular Lie algebras . . . . . . . . . . . . . . . . . . . . . . . . 1738

References . . . . . . . . . . . . . . . . . . . . . . 1738

Marco Freibert

freibert@math.uni-kiel.de

Andrew Swann

swann@math.au.dk

1 Mathematisches Seminar, Christian-Albrechts-Universität zu Kiel, Ludewig-Meyn-Strasse 4, 24098 Kiel, Germany

2 Department of Mathematics, and DIGIT, Aarhus University, Ny Munkegade 118, Bldg 1530, 8000 Aarhus C, Denmark 


\section{Introduction}

Recent years have seen a rising interest in non-Kähler Hermitian manifolds $(M, g, J)$. The most studied case is strong Kähler with torsion (SKT) geometry, which may be characterized by $\partial \bar{\partial} \omega=0$, where $\omega$ is the associated fundamental two-form. An equivalent description is obtained if one uses the Bismut connection $\nabla^{B}$ on $(M, g, J)$, which is the unique connection with $\nabla^{B} g=0, \nabla^{B} J=0$ and the associated torsion tensor $T^{B}$ totally skew-symmetric (after lowering one index). Then $T^{B}=-J^{*} d \omega$ and $(M, g, J)$ is SKT if and only if $d T^{B}=0$.

The latter property is used in physics in the context of supersymmetric theories, cf. for example [15,17,21]. These structures also appear in generalized Kähler geometry [3,6,10,15], and in two complex dimensions they are "standard" in the sense of Gauduchon [14], i.e. any conformal class of any Hermitian metric on a compact complex surface admits an SKT metric. Moreover, any compact Lie group possesses a left-invariant SKT structure, see e.g. [18].

However, in higher dimensions, the existence of an SKT metric is not automatically guaranteed anymore. For example, looking at the invariant case on nilpotent Lie groups, only 4 out of the 34 six-dimensional nilpotent Lie algebras admit (non-Kähler) SKT structures [11]. Also the eight-dimensional nilpotent case has been classified in [7], and in both cases all nilpotent SKT Lie algebras are two-step nilpotent, leading to the question whether this is a general feature in arbitrary dimensions [13].

A more general class of Lie algebras that so far only has partially been studied, is the solvable one. Here, a full classification has only been achieved in dimension four by the second author in cooperation with T. B. Madsen [18]. This reflects the much more complicated classification of solvable Lie algebras. In fact, in dimension six, the classification of all solvable Lie algebras is already very long and complicated [20,23] and in higher dimensions there is no classification at all, nor is there hope that there will be a full list in the future. Hence, so far, only classifications of solvable SKT Lie algebras under severe extra conditions in dimensions six and higher are known. For example, [9] classifies the six-dimensional solvable Lie algebras admitting an SKT structure and a non-zero holomorphic (3,0)-form, and in [8] it is shown that no complex solvable Lie group may admit an invariant SKT structure. Moreover, [4] provides a classification of the almost Abelian Lie algebras admitting an SKT structure, cf. also [10] for a more explicit list in the six-dimensional case.

In this article, we determine, for various natural classes of two-step solvable (almost Hermitian) Lie algebras, the ones which are SKT. This includes, in particular, also a new proof for the classification in the almost Abelian case leading to a more compact description of the almost Abelian Lie algebras admitting an SKT structure in Corollary 4.7. Moreover, our results eventually lead to a classification of all but one class of six-dimensional two-step solvable Lie algebras admitting an SKT structure in Theorem 7.1.

To obtain the classification results, we apply the shear construction introduced in our joint paper [12]. This construction is a generalisation of the twist construction invented by the second-named author in [22]. The twist looks at double fibrations of the form $M \leftarrow P \rightarrow W$ of two principal $A$-bundles with commuting $A$-actions, $A$ being a connected Abelian Lie group, and transfers $A$-invariant tensor fields from $M$ to $W$ by requiring that their pullbacks to $W$ agree on the horizontal distribution in $P$. The principal $A$-bundles and all other data may be obtained by "twist data" on $M$ and the twist is well-adapted to nilpotent Lie groups and nilmanifolds in the sense that any nilmanifold may be constructed from a torus by several twists increasing the nilpotent step length of the associated nilpotent Lie group by one with each step. Note that the second author used the twist construction in the above mentioned 
paper [22] to construct new examples of compact simply-connected SKT manifolds out of well-known ones.

The shear construction now replaces the group actions of the twist by flat connections and the principal bundles by certain types of submersions and is well-adapted to solvable Lie groups in the sense that any simply-connected solvable Lie group may be obtained from $\mathbb{R}^{n}$ by consecutive shears increasing the solvable step length by one. Again, the shear construction can be completely encoded by "shear data" on $M$ and so any two-step solvable SKT Lie algebra can be obtained by one shear from the standard flat (left-invariant) Kähler structure on $\mathbb{R}^{n}$ and appropriate shear data on $\mathbb{R}^{n}$.

Let us now give a more detailed overview of the results in this paper and outline its organisation.

In Sect. 2, we recall the shear construction on arbitrary Lie algebras and give a full description of the possible shear data on Abelian Lie algebras.

Section 3 first derives the general conditions for shear data on $\mathbb{R}^{2 n}$ to shear the flat Kähler structure to an SKT-structure. For this, we decompose $\mathfrak{g}$ into $\mathfrak{g}_{J}^{\prime} \oplus \mathfrak{g}_{r}^{\prime} \oplus J \mathfrak{g}_{r}^{\prime} \oplus U_{J}$, where $\mathfrak{g}_{J}^{\prime}:=\mathfrak{g}^{\prime} \cap J \mathfrak{g}^{\prime}, \mathfrak{g}_{r}^{\prime}$ is the orthogonal complement of $\mathfrak{g}_{J}^{\prime}$ in $\mathfrak{g}^{\prime}$ and $U_{J}$ is the orthogonal complement of $\mathfrak{g}_{J}^{\prime} \oplus \mathfrak{g}_{r}^{\prime} \oplus J \mathfrak{g}_{r}^{\prime}$ in $\mathfrak{g}$. Afterwards, we derive some general consequences on the different components of the shear data with respect to this splitting. In particular, we show that ad $\left(J \mathfrak{g}_{r}^{\prime}\right)$ preserves $\mathfrak{g}_{J}^{\prime}$ and is diagonalisable over the complex on this subspace.

Section 4 deals with the case $U_{J}=0$, which is equivalent to $\mathfrak{g}^{\prime} \oplus J \mathfrak{g}^{\prime}=\mathfrak{g}$. We derive the general form of a two-step solvable SKT Lie algebra of this form and then deal individually with the cases $\operatorname{codim}\left(\mathfrak{g}^{\prime}\right)=1$ and $\operatorname{codim}\left(\mathfrak{g}^{\prime}\right)=2$. The first case is the almost Abelian one and we give a full classification of all SKT almost Abelian Lie algebras in Theorem 4.5 as well as an easy characterisation of the almost Abelian Lie algebras admitting an SKT structure in Corollary 4.7. The second case may equivalently be described as $\mathfrak{g}^{\prime}$ being of codimension two and $\mathfrak{g}^{\prime}$ not being $J$-invariant. We classify all such two-step solvable SKT Lie algebras in Theorem 4.10. In both cases, we note that some of the resulting algebras are unimodular.

In Sect. 5, we consider the case $\mathfrak{g}_{J}^{\prime}=\{0\}$, so $\mathfrak{g}^{\prime}$ is totally real. Note that then the complex structure of an SKT structure is Abelian and classify such SKT Lie algebras in Theorem 5.5 up to some minor remaining equation. In particular, we show that then the Lie algebra is of the form $r \mathfrak{a f f} \mathfrak{R}_{\mathbb{R}} \oplus \mathfrak{h}$ for some nilpotent Lie algebra $\mathfrak{h}, r:=\operatorname{dim}\left(\left[\mathfrak{g}^{\prime}, J \mathfrak{g}^{\prime}\right]\right)$. If $\left[\mathfrak{g}^{\prime}, J \mathfrak{g}^{\prime}\right]$ is of codimension $\ell$ at most two in $\mathfrak{g}^{\prime}$, we are also able to solve the remaining equation and $\mathfrak{g}$ is isomorphic to $r \mathfrak{a f f} \mathfrak{R}_{\mathbb{R}} \oplus \mathbb{R}^{2 n-2 r}(\ell=0), r \mathfrak{a f f} \mathfrak{R}_{\mathbb{R}} \oplus \mathfrak{h}_{3} \oplus \mathbb{R}^{2 n-2 r-3}(\ell=1)$ or to $r \mathfrak{a f f} \mathfrak{f}_{\mathbb{R}} \oplus 2 \mathfrak{h}_{3} \oplus \mathbb{R}^{2 n-2 r-6}(\ell=2)$, respectively. We use our results to obtain a classification of all SKT Lie algebras with one-dimensional commutator or with two-dimensional commutator which is not $J$-invariant. The corresponding Lie algebras are isomorphic to aff $\mathfrak{f}_{\mathbb{R}} \oplus \mathbb{R}^{2 n-2}$, $\mathfrak{h}_{3} \oplus \mathbb{R}^{2 n-3}$ or to $2 \mathfrak{a f f} \mathfrak{f}_{\mathbb{R}} \oplus \mathbb{R}^{2 n-4}, \mathfrak{a} \mathfrak{f} \mathfrak{f}_{\mathbb{R}} \oplus \mathfrak{h}_{3} \oplus \mathbb{R}^{2 n-5}, 2 \mathfrak{h}_{3} \oplus \mathbb{R}^{2 n-6}$, respectively.

Section 6 determines all SKT Lie algebras with $\operatorname{dim}\left(\mathfrak{g}^{\prime}\right)=2$ and $\mathfrak{g}^{\prime}$ being $J$-invariant in Theorem 6.5. The associated Lie algebra is isomorphic either to $\mathfrak{r}_{3,0}^{\prime} \oplus \mathbb{R}^{2 n-3}$ or to $\mathfrak{h} \oplus \mathbb{R}^{2 n-2 k}$ with a six- or eight-dimensional (i.e. $k \in\{3,4\}$ ) nilpotent SKT Lie algebra. Hence, we obtain a full list of these Lie algebras using the results in [11] and [7].

Finally, in Sect. 7 we put our previous results together, solve one additional case in Theorem 7.5 (for $\operatorname{dim}(\mathfrak{g})=6, \operatorname{dim}\left(\mathfrak{g}^{\prime}\right)=3, \mathfrak{g}^{\prime} \neq \mathfrak{g}_{r}^{\prime}$ ) and so are able to give a full classification of six-dimensional two-step solvable Lie algebras admitting an SKT structure in Theorem 7.1 up to one particular case, where we write down the resulting equations. 


\section{Two-step solvable algebras}

The general shear construction [12], is motivated by and specialises to constructions of Lie groups $H$ from left-invariant data on a Lie group $G$ of the same dimension. The construction is set-up so that if $G$ is solvable, then $H$ is also solvable with in general step length one greater than that of $G$. The construction may be understood purely at the algebraic level, using left-invariant shear data, as follows.

Let $\mathfrak{g}$ be the Lie algebra of $G$. Suppose $\mathfrak{a}_{G}$ and $\mathfrak{a}_{P}$ are Abelian Lie algebras of the same dimension and we have $(\xi, a, \omega, \gamma, \eta)$, where $\gamma \in \mathfrak{g}^{*} \otimes \mathfrak{g l}\left(\mathfrak{a}_{G}\right), \eta \in \mathfrak{g}^{*} \otimes \mathfrak{g l}\left(\mathfrak{a}_{P}\right), \xi: \mathfrak{a}_{G} \rightarrow \mathfrak{g}$ a Lie algebra monomorphism, $a: \mathfrak{a}_{G} \rightarrow \mathfrak{a}_{P}$ a Lie algebra isomorphism and a two-form $\omega \in \Lambda^{2} \mathfrak{g}^{*} \otimes \mathfrak{a}_{P}$ with values in $\mathfrak{a}_{P}$. Then $(\xi, a, \omega, \gamma, \eta)$ defines left-invariant shear data on $\mathfrak{g}$ if and only $\xi\left(\mathfrak{a}_{G}\right)$ is an ideal in $\mathfrak{g}$ and the following conditions are satisfied:

(Sa) $\xi^{*} \omega=0$,

(Sb) $d \omega+\eta \wedge \omega=0$,

(Sc) $\left.\eta=a \gamma a^{-1}-(\xi\lrcorner \omega\right) a^{-1}$,

(Sd) $\gamma(\xi Y)=0$ for all $Y \in \mathfrak{a}_{G}$,

(Se) $\xi \circ \gamma(X)=\operatorname{ad}(X) \circ \xi$ for all $X \in \mathfrak{g}$, and

(Sf) $d \eta+\eta \wedge \eta=0$.

The shear $H$ of $G$ then has Lie algebra $\mathfrak{h}=\mathfrak{p} / \stackrel{\xi}{\xi}\left(\mathfrak{a}_{G}\right)$, where $\mathfrak{p}$ is the extension of $\mathfrak{g}$ by $\mathfrak{a}_{P}$ via the two-form $\omega$ and $\stackrel{\xi}{\xi}: \mathfrak{a} \rightarrow \mathfrak{p}$ is a lift of $\xi$. More precisely, as a vector space $\mathfrak{p}=\mathfrak{g} \oplus \mathfrak{a}_{P}$ with $\mathfrak{a}_{P}$ an Abelian ideal, $[X, Y]_{\mathfrak{p}}=[X, Y]_{\mathfrak{g}}-\omega(X, Y),[X, Z]_{\mathfrak{p}}=\eta(X) Z$. The lift $\stackrel{\xi}{\xi}$ is given as $\tilde{\xi}+\rho \circ a$, where $\rho: \mathfrak{a}_{P} \rightarrow \mathfrak{p}$ is the inclusion and $\tilde{\xi}: \mathfrak{a}_{G} \rightarrow \mathfrak{p}$ is the horizontal lift of $\xi$ with respect to the flat connection on $E=G \times \mathfrak{a}_{G}$ defined by $\gamma$.

The above construction and data simplifies considerably if we start with $G$ Abelian, as we will now explain. So take $\mathfrak{g}=\mathbb{R}^{N}$ for some $N$. Then as $\xi$ is injective, we may assume that $\mathfrak{a}=\mathfrak{a}_{G}$ is a Lie subalgebra of $\mathfrak{g}=\mathbb{R}^{N}$, so an arbitrary subspace, and that $\xi=$ inc is the inclusion. Next, since $a$ is invertible, we may take $\mathfrak{a}_{P}=\mathfrak{a}$ and $a=\mathrm{id}_{\mathfrak{a}}$, the identity map. It turns out that given the form $\omega$ the remaining pieces of shear data $\gamma$ and $\eta$ are uniquely determined. To explain this, let $U$ be a vector space complement to $\mathfrak{a}$ in $\mathfrak{g}$ :

$$
\mathfrak{g}=\mathfrak{a}+U .
$$

Then

$$
\Lambda^{2} \mathfrak{g}^{*}=\Lambda^{2} \mathfrak{a}^{*} \oplus U^{*} \wedge \mathfrak{a}^{*} \oplus \Lambda^{2} U^{*},
$$

and we correspondingly decompose

$$
\omega=\omega_{-1}+\omega_{0}+\omega_{1},
$$

with $\omega_{-1} \in \Lambda^{2} \mathfrak{a}^{*} \otimes \mathfrak{a}, \omega_{0} \in\left(U^{*} \wedge \mathfrak{a}^{*}\right) \otimes \mathfrak{a} \subset U^{*} \otimes$ End $\mathfrak{a}$ and $\omega_{1} \in \Lambda^{2} U^{*} \otimes \mathfrak{a}$.

Lemma 2.1 For $\mathfrak{g}=\mathbb{R}^{N}$ Abelian, (inc, $\mathrm{id}_{\mathfrak{a}}, \omega=\omega_{-1}+\omega_{0}+\omega_{1}, \gamma, \eta$ ) defines left-invariant shear data if and only if $\omega_{-1}=0, \gamma=0, \eta=-\omega_{0}$ and

$$
\mathcal{A}(\omega(\omega(\cdot, \cdot), \cdot))=0 \text {, }
$$

where $\mathcal{A}$ is the anti-symmetrisation map over all arguments.

Proof We need to check conditions $(\mathrm{Sa})-(\mathrm{Sf})$. The first condition $(\mathrm{Sa})$ is exactly $\omega_{-1}=0$. Now as $\mathfrak{g}$ is Abelian condition (Se), says inco $\gamma(X)=\operatorname{ad}(X) \circ$ inc $=0$ for all $X \in \mathfrak{g}=\mathbb{R}^{N}$, so $\gamma=0$. This implies condition ( $\mathrm{Sd}$ ) is automatically satisfied and condition $(\mathrm{Sc})$ is equivalent 
to $\eta=-$ inc $\lrcorner \omega=-\omega_{0} \in U^{*} \otimes$ End $\mathfrak{a}$. Considering condition (Sb), we get $0=d \omega+\eta \wedge \omega=$ $-\omega_{0} \wedge \omega$. But if we now regard $\omega_{0}$ as an element of $\left(U^{*} \wedge \mathfrak{a}^{*}\right) \otimes \mathfrak{a} \subseteq \Lambda^{2} \mathfrak{g}^{*} \otimes \mathfrak{a}$, then this condition reads $\mathcal{A}\left(\omega_{0}(\omega(\cdot, \cdot), \cdot)\right)=0$. As $\omega_{1}(\omega(\cdot, \cdot), \cdot)=0$, this is equivalent to (2.1).

Finally, we need to consider condition (Sf), i.e. $0=d \eta+\eta \wedge \eta=\eta \wedge \eta$. If we regard $\omega_{0}$ as an element of $\left(U^{*} \wedge \mathfrak{a}^{*}\right) \otimes \mathfrak{a} \subseteq \Lambda^{2} \mathfrak{g}^{*} \otimes \mathfrak{a}$, then this condition is equivalent to $\mathcal{A}\left(\omega_{0}\left(\omega_{0}(\cdot, \cdot), \cdot\right)\right)=0$, which is automatic under $(2.1)$.

As all two-step solvable algebras are obtained as shears of Abelian algebras [12, Proposition 2.5], we see that shear data for such a two-step algebra consists of $\mathfrak{g}=\mathbb{R}^{N}$, a subalgebra $\mathfrak{a} \leq \mathfrak{g}$ and $\omega=\omega_{0}+\omega_{1} \in\left(U^{*} \wedge \mathfrak{a}^{*}+\Lambda^{2} U^{*}\right) \otimes \mathfrak{a}$ satisfying (2.1).

Definition 2.2 We call ( $\mathfrak{a}, \omega)$ two-step shear data on $\mathfrak{g}=\mathbb{R}^{N}$, with $\mathfrak{a}$ the shearing algebra and $\omega$ the shearing form. If $\omega \in\left(U^{*} \wedge \mathfrak{a}^{*}+\Lambda^{2} U^{*}\right) \otimes \mathfrak{a}$ does not necessarily satisfy (2.1), then we call $(\mathfrak{a}, \omega)$ pre-shear data.

\section{Data for two-step solvable SKT algebras}

The shear construction becomes a useful computational tool in the presence of geometric structures. In general, provided they satisfy an invariance property with respect to $\mathfrak{a}_{G}$, they may be lifted horizontally to $\mathfrak{p}$ and then descend to the shear $\mathfrak{h}=\mathfrak{p} / \mathfrak{\xi}_{\mathfrak{\xi}} \mathfrak{a}_{G}$; starting with a tensor $\alpha$, we denote the resulting tensor on $\mathfrak{h}$ by $\alpha_{\mathfrak{h}}$ and say that $\alpha$ and $\alpha_{\mathfrak{h}}$ are $\mathcal{H}$-related, where $\mathcal{H}$ refers the horizontal distribution of a certain connection on $\mathfrak{p}$. In [12], we provided a number of simple formulae for computing various derived properties of $\alpha_{\mathfrak{h}}$ in terms of properties of $\alpha$ on $\mathfrak{g}$ and the shear data.

Let $(\mathfrak{a}, \omega)$ be two-step shear data on $\mathfrak{g}=\mathbb{R}^{N}$ and assume that $N=2 n$ for some $n \in \mathbb{N}$. We equip $\mathfrak{g}=\mathbb{R}^{2 n}$ with the standard flat Kähler structure $(g, J, \sigma=g(J \cdot, \cdot))$. We will determine the conditions for such a shear $\mathfrak{h}$ to be SKT (strong Kähler with torsion) if we start with the standard structure on $\mathbb{R}^{2 n}$.

First recall, that an SKT structure on an algebra $\mathfrak{h}$ consists of a Hermitian structure $\left(g_{\mathfrak{h}}, J_{\mathfrak{h}}, \sigma_{\mathfrak{h}}\right)$, in particular $J_{\mathfrak{h}}$ is integrable, such that the torsion three-form $c_{\mathfrak{h}}=-J_{\mathfrak{h}}^{*} d \sigma_{\mathfrak{h}}$ is closed. This then defines a left-invariant SKT structure on the group $H$, and conversely each such left-invariant structure arises this way. Note that any Kähler structure is SKT, and that any almost Hermitian structure on the Abelian algebra $\mathfrak{g}=\mathbb{R}^{2 n}$ is Kähler.

Lemma 3.1 Let $(\mathfrak{a}, \omega)$ be two-step shear data on $\mathfrak{g}=\mathbb{R}^{2 n}$. Let $(g, J, \sigma)$ be a flat Kähler structure on $\mathfrak{g}=\mathbb{R}^{2 n}$. Then the induced almost Hermitian geometry $\left(g_{\mathfrak{h}}, J_{\mathfrak{h}}, \sigma_{\mathfrak{h}}\right)$ on the shear $\mathfrak{h}$ is SKT if and only if the following two equations hold on $\mathfrak{g}$ :

$$
\begin{aligned}
& J^{*} \omega=\omega-J \circ J . \omega, \\
& \mathcal{A}\left(g\left(J^{*} \omega(\cdot, \cdot), \omega(\cdot, \cdot)\right)+2 g\left(J^{*} \omega(\omega(\cdot, \cdot), \cdot), \cdot\right)\right)=0,
\end{aligned}
$$

where $(J . \omega)(X, Y)=-\omega(J X, Y)-\omega(X, J Y)$, and $\mathcal{A}$ is anti-symmetrisation over all arguments. Furthermore, any two-step solvable SKT algebra $\mathfrak{h}$ may be obtained as a shear of a flat Kähler structure on $\mathfrak{g}=\mathbb{R}^{2 n}$ in this way.

Proof As $\gamma=0$, the invariance conditions in [12, Sects. 3.2-3.3] are just that the tensors are left-invariant on $G$. The condition for $J_{\mathfrak{h}}$ to be integrable is equivalent to (3.1) by [12, Proposition 3.13], since $J$ is integrable. If $\alpha \in \Lambda^{k} \mathfrak{g}^{*}$, then as $\mathfrak{g}$ is Abelian, we have $d \alpha=0$. It follows from [12, Corollary 3.11], that $d \alpha_{\mathfrak{h}} \in \Lambda^{k+1} \mathfrak{h}^{*}$ is $\mathcal{H}$-related to the differential form 
$\left.d_{\mathfrak{h}} \alpha:=-(\xi\lrcorner \alpha\right) \wedge \omega \in \Lambda^{k+1} \mathfrak{g}^{*}$, where $\xi=$ inc $: \mathfrak{a} \rightarrow \mathfrak{g}$ acts on the values of $\omega$. In other words, $d_{\mathfrak{h}} \alpha=-\mathcal{A}(\alpha(\omega(\cdot, \cdot), \cdot, \ldots, \cdot))$. We thus find the three-form on $c_{\mathfrak{g}}$ on $\mathfrak{g}$ that is $\mathcal{H}$-related to the torsion three-form $c_{\mathfrak{h}}=-J_{\mathfrak{h}}^{*} d \sigma_{\mathfrak{h}}$ on $\mathfrak{h}$ is given by

$$
\left.c_{\mathfrak{g}}=-J^{*} d_{\mathfrak{h}} \sigma=J^{*}((\xi\lrcorner \sigma) \wedge \omega\right)=-J^{*} \omega \wedge \xi^{b}=-\mathcal{A}\left(g\left(J^{*} \omega(\cdot, \cdot), \cdot\right)\right) .
$$

Now the differential $d c_{\mathfrak{h}}$ is $\mathcal{H}$-related to $d_{\mathfrak{g}} c_{\mathfrak{g}}$ is given by

$$
\begin{aligned}
d_{\mathfrak{g}} c_{\mathfrak{g}} & \left.=-\omega \wedge(\xi\lrcorner c_{\mathfrak{g}}\right)=-\mathcal{A}\left(c_{\mathfrak{g}}(\omega(\cdot, \cdot), \cdot, \cdot)\right) \\
& =\frac{1}{3} \mathcal{A}\left(g\left(J^{*} \omega(\cdot, \cdot), \omega(\cdot, \cdot)\right)+2 g\left(J^{*} \omega(\omega(\cdot, \cdot), \cdot), \cdot\right)\right) .
\end{aligned}
$$

So $d c_{\mathfrak{h}}=0$ is Eq. (3.2).

Conversely, if $\mathfrak{h}$ is two-step solvable, then there is some left-invariant shear to $\mathfrak{g}=\mathbb{R}^{2 n}$, where $2 n=\operatorname{dim} \mathfrak{h}$, by [12, Proposition 2.5]. Now the SKT structure on $\mathfrak{h}$ induces is $\mathcal{H}$-related to an almost Hermitian structure on $\mathfrak{g}$. But $\mathfrak{g}$ is Abelian, so this structure is Kähler, and the structure on $\mathfrak{h}$ comes from the above construction.

Definition 3.2 Two-step shear data (a, $\omega$ ) satisfying (3.1) and (3.2) will be called two-step SKT shear data.

Notation We set

$$
\begin{aligned}
v_{1} & :=v_{1}(\omega):=\mathcal{A}\left(g\left(J^{*} \omega(\cdot, \cdot), \omega(\cdot, \cdot)\right)\right) \in \Lambda^{4} \mathfrak{g}^{*}, \\
v_{2} & :=v_{2}(\omega):=\mathcal{A}\left(g\left(J^{*} \omega(\omega(\cdot, \cdot), \cdot), \cdot\right)\right) \in \Lambda^{4} \mathfrak{g}^{*}, \\
v & :=v(\omega):=v_{1}(\omega)+2 v_{2}(\omega) .
\end{aligned}
$$

for two-step shear data $(\mathfrak{a}, \omega)$. Note that (3.2) holds if and only if $v=0$.

Summarizing, to find two-step solvable SKT algebras we need to solve Eqs. (2.1), (3.1) and (3.2). To do this, let us reformulate the conditions using information from both the data $(\mathfrak{a}, \omega)$ and the Kähler structure $(g, J, \sigma)$ on $\mathfrak{g}=\mathbb{R}^{2 n}$. For $V \leq \mathfrak{g}$ a linear subspace, we write

$$
V_{J}=V \cap J V \text { and } V_{r}=\left(V_{J}\right)^{\perp} \cap V .
$$

We say that $V$ is complex if $V=V_{J}$ or totally real for $V=V_{r}$. Putting $U:=J \mathfrak{a}_{r} \oplus\left(\mathfrak{a} \oplus J \mathfrak{a}_{r}\right)^{\perp}$, we thus have

$$
\mathfrak{g}=\mathfrak{a} \oplus U=\mathfrak{a}_{J} \oplus \mathfrak{a}_{r} \oplus U_{J} \oplus U_{r}
$$

We note that

$$
U_{r}=J \mathfrak{a}_{r}, U_{J}=\left(\mathfrak{a} \oplus J \mathfrak{a}_{r}\right)^{\perp} .
$$

In this setting, it is very natural to decompose also $\omega_{0} \in\left(U^{*} \wedge \mathfrak{a}^{*}\right) \otimes \mathfrak{a}$ and $\omega_{1} \in \Lambda^{2} U^{*} \otimes \mathfrak{a}$ further, using the decompositions $\mathfrak{a}=\mathfrak{a}_{J} \oplus \mathfrak{a}_{r}, U=U_{J} \oplus U_{r}$ and the associated dual decompositions. We then write $\left(\omega_{0}\right)_{r J}^{J}$ for the $U_{r}^{*} \otimes \mathfrak{a}_{J}^{*} \otimes \mathfrak{a}_{J}$-component of $\omega_{0}$, etc. Similarly, we write $\left(\omega_{1}\right)_{J r}^{r}$ for the $U_{J}^{*} \otimes U_{r}^{*} \otimes \mathfrak{a}_{r} \cong U_{J}^{*} \wedge U_{r}^{*} \otimes \mathfrak{a}_{r}$-part of $\omega_{1}$, etc.

It turns out to be useful to consider the endomorphism $\left(\omega_{0}\right)(Y, \cdot) \in \operatorname{End}(\mathfrak{a})$ for $Y \in U_{r}$. More conveniently, we define

$$
A_{X}:=-\omega_{0}(J X, \cdot) \in \operatorname{End}(\mathfrak{a}), \quad \text { for } X \in \mathfrak{a}_{r},
$$


and decompose this further as

$$
\begin{aligned}
A_{X} & =F_{X}+G_{X}+H_{X}+K_{X} \\
& \in \operatorname{End}\left(\mathfrak{a}_{r}\right)+\operatorname{Hom}\left(\mathfrak{a}_{J}, \mathfrak{a}_{r}\right)+\operatorname{Hom}\left(\mathfrak{a}_{r}, \mathfrak{a}_{J}\right)+\operatorname{End}\left(\mathfrak{a}_{J}\right),
\end{aligned}
$$

so $F_{X}=-\left(\omega_{0}\right)_{r r}^{r}(J X, \cdot)$, etc. Associated to $F_{X}$ and $H_{X}$, we have bilinear maps

$$
\begin{aligned}
& f: \mathfrak{a}_{r} \otimes \mathfrak{a}_{r} \rightarrow \mathfrak{a}_{r} \\
& f(X, Y):=F_{X}(Y) \\
& h: \mathfrak{a}_{r} \otimes \mathfrak{a}_{r} \rightarrow \mathfrak{a}_{J} \\
& h(X, Y):=H_{X}(Y)
\end{aligned}
$$

for $X, Y \in \mathfrak{a}_{r}$.

Lemma 3.3 The shearing form $\omega$ satisfies the two-step integrability equation (3.1) if and only if

(i) $G_{X}=0$ for all $X \in \mathfrak{a}_{r}$,

(ii) $\left[J, K_{X}\right]=0$ for all $X \in \mathfrak{a}_{r}$,

(iii) $f \in \mathfrak{a}_{r}^{*} \otimes \mathfrak{a}_{r}^{*} \otimes \mathfrak{a}_{r}$ is symmetric in its $\mathfrak{a}_{r}^{*}$-factors,

(iv) $\left(\omega_{1}\right)_{r r}^{r}=0$,

(v) $J^{*}\left(\omega_{1}\right)_{r r}^{J}=-2 J \circ \mathcal{A}(h)$,

(vi) $\left(\omega_{0}\right)_{J r}^{r}=J^{*}\left(\omega_{1}\right)_{J r}^{r}$,

(vii) $\left(\omega_{1}\right)_{J r}^{J}(J X, J Y)-J\left(\left(\omega_{1}\right)_{J r}^{J}(X, J Y)\right)=\left(\omega_{0}\right)_{J r}^{J}(X, Y)+J\left(\left(\omega_{0}\right)_{J r}^{J}(J X, Y)\right)$ for all $X \in U_{J}$ and all $Y \in \mathfrak{a}_{r}$,

(viii) $\left(\omega_{0}\right)_{J J}^{r}$ is of type $(1,1)$ and $J^{*}\left(\omega_{0}\right)_{J J}^{J}=\left(\omega_{0}\right)_{J J}^{J}-J\left(J .\left(\omega_{0}\right)_{J J}^{J}\right)$, and

(ix) $\left(\omega_{1}\right)_{J J}^{r}$ is of type $(1,1)$ and $J^{*}\left(\omega_{1}\right)_{J J}^{J}=\left(\omega_{1}\right)_{J J}^{J}-J\left(J .\left(\omega_{1}\right)_{J J}^{J}\right)$.

Proof First of all, observe that if (3.1) holds for $X, Y \in \mathfrak{g}=\mathbb{R}^{2 n}$, then this equation is also true when evaluated on the pairs $(J X, Y),(X, J Y)$ and $(J X, J Y)$. Surely, all tensors in (3.1) are additionally anti-symmetric. Moreover, as $\left.\omega\right|_{\Lambda^{2} \mathfrak{a}^{*}}=0,(3.1)$ is trivially satisfied for $X, Y \in \mathfrak{a}_{J}$, as then both sides of that equation are zero. Hence, we are left with the following cases, which correspond directly to the conditions of the Lemma: (a) $X \in \mathfrak{a}_{r}$, $Y \in \mathfrak{a}_{J}$ gives (i) and (ii), (b) $X, Y \in \mathfrak{a}_{r}$ gives (iii), (iv) and (v), (c) $X \in \mathfrak{a}_{r}, Y \in U_{J}$ leads to (vi) and (vii), (d) $X \in \mathfrak{a}_{J}, Y \in U_{J}$ gives (viii), and finally (e) $X, Y \in U_{J}$ leads to (ix).

Using Lemma 3.3 (i) and (iv), $\mathfrak{a}_{J} \perp\left(\mathfrak{a}_{r} \oplus U_{r}\right)$ and $U_{J} \perp \mathfrak{a}$, one observes

Lemma 3.4 Let $(\mathfrak{a}, \omega)$ be two-step shear data. Then

(i) $v_{1}(\omega)=0$ on $\Lambda^{4} \mathfrak{a}+\Lambda^{4}\left(\mathfrak{a}_{J} \oplus U_{r}\right)+\Lambda^{3} \mathfrak{a}_{J} \wedge \mathfrak{g}$,

(ii) $v_{2}(\omega)=0$ on $\Lambda^{4} \mathfrak{a}+\Lambda^{4}\left(\mathfrak{a}_{J} \oplus U_{r}\right)+\Lambda^{3} \mathfrak{a}_{r} \wedge U_{r}+\Lambda^{4} U_{J}$, and

(iii) $v(\omega)=0$ on $\Lambda^{4} \mathfrak{a}+\Lambda^{4}\left(\mathfrak{a}_{J} \oplus U_{r}\right)$.

Next, let us look at (2.1). We observe that, evaluated on $X_{1}, X_{2} \in U, Y \in \mathfrak{a}$, the equation is satisfied if and only if the endomorphisms $\omega_{0}\left(X_{1}, \cdot\right), \omega_{0}\left(X_{2}, \cdot\right)$ of $\mathfrak{a}$ commute. Thus, (2.1) is true on $\Lambda^{2} U_{r} \wedge \mathfrak{a}$ if and only if for all $X_{1}, X_{2} \in \mathfrak{a}_{r}$ we have $\left[A_{X_{1}}, A_{X_{2}}\right]=0$, which is equivalent to $\left[K_{X_{1}}, K_{X_{2}}\right]=0, K_{X_{1}} H_{X_{2}}+H_{X_{1}} F_{X_{2}}=K_{X_{2}} H_{X_{1}}+H_{X_{1}} F_{X_{1}}$ and $\left[F_{X_{1}}, F_{X_{2}}\right]=0$ for all $X_{1}, X_{2} \in \mathfrak{a}_{r}$, the latter equation also may be reformulated, due to $f$ being symmetric, as $f(f(\cdot, \cdot), \cdot)$ being totally symmetric.

The other parts of (2.1) are more difficult and we will evaluate them explicitly below in the different cases that we are going to consider in more detail. Thus, let us look at (3.2), i.e. at $v(\omega)$. Here, the $\Lambda^{2} \mathfrak{a}_{J} \wedge \mathfrak{a}_{r} \wedge U_{r}$-part of $v(\omega)$ gives equations which solely contain different components of $A_{X}$. A straightforward computation using the fact that $f$ is symmetric, that $K_{X}$ commutes with $J$ and all other $K_{X^{\prime}}$ gives us the second part of 
Lemma 3.5 Let $\mathfrak{a}$ be a subspace of $\mathbb{R}^{2 n}$, let $\omega \in \Lambda^{2}\left(\mathbb{R}^{2 n}\right)^{*} \otimes \mathfrak{a}$ with $\left.\omega\right|_{\Lambda^{2} \mathfrak{a}^{*}}=0$ fulfilling (3.1) and let $(g, J)$ be the standard Kähler structure on $\mathbb{R}^{2 n}$. Then, with the decompositions and notations from above, the two-form $\omega$ satisfies (2.1) on $\Lambda^{2} U_{r} \wedge \mathfrak{a}$ and (3.2) on $\Lambda^{2} \mathfrak{a}_{J} \wedge \mathfrak{a}_{r} \wedge U_{r}$ if and only if for all $X, W \in \mathfrak{a}_{r}$ all of the following conditions are valid

$$
\begin{array}{r}
{\left[K_{X}, K_{W}\right]=0, \quad\left[F_{X}, F_{W}\right]=0,} \\
K_{X} H_{W}+H_{X} F_{W}=K_{W} H_{X}+H_{W} F_{X}
\end{array}
$$

and

$$
K_{X}^{T} K_{W}+K_{X} K_{W}+K_{f(X, W)}
$$

is g-antisymmetric. Here, the superscript " $T$ " denotes the g-transpose of an endomorphism of $\mathfrak{a}_{J}$.

Before we specialize to the different cases, we will use (3.5) and (3.6) to simplify the form of the endomorphisms $K_{X}$ of $\mathfrak{a}_{J}$ with respect to a certain basis of $\mathfrak{a}_{J}$.

For this, note that by Lemma 3.3(ii) these endomorphisms may be considered as complex endomorphisms on the complex vector space $\left(\mathfrak{a}_{J}, J\right)$. From now on, we will often consider $\mathfrak{a}_{J}$ as a complex vector space and note that the scalar multiplication satisfies $(a+i b) X=$ $a X+b J X$ for $a, b \in \mathbb{R}$ and $X \in \mathfrak{a}_{J}$. Even more, we will identify the real Hermitian vector space $\left(\mathfrak{a}_{J}, g, J\right)$ with the complex unitary vector space $\left(\mathfrak{a}_{J}, g+i \omega\right)$.

The rest of this section aims at showing that all complex endomorphisms $K_{X}$ are simultaneously complex diagonalizable by a unitary basis. For this, note that Lemma 3.5 tells us that all these endomorphisms commute and so we need to show that all these endomorphisms are diagonalizable. This will eventually follow from (3.6) and for this it turns out to be useful to find a good basis $\left(v_{1}, \ldots, v_{m}\right)$ of $\mathfrak{a}_{r}$ in which $f\left(v_{i}, v_{i}\right)$ is not too complicated.

Lemma 3.6 Let $(V,\langle\cdot, \cdot\rangle)$ be an inner product space of dimension $n$ and let $f: S^{2} V \rightarrow$ $V$ be linear. Then there exists an orthonormal basis $\left(v_{1}, \ldots, v_{n}\right)$ of $V$ with $f\left(v_{i}, v_{i}\right) \in$ $\operatorname{span}\left(v_{1}, \ldots, v_{i}\right)$ for all $i=1, \ldots, n$.

Proof The proof is by induction on $n$. The case $n=1$ holds trivially.

For $n>1$, we first show that there is some unit vector $v_{1} \in V$ with $f\left(v_{1}, v_{1}\right) \in \operatorname{span}\left(v_{1}\right)$. If there is some $v_{1} \in V \backslash\{0\}$ with $f\left(v_{1}, v_{1}\right)=0$, we are done. Otherwise, let $S^{n-1}=$ $\{v \in V:\|v\|=1\}$ be the unit sphere and consider the function $F: S^{n-1} \rightarrow S^{n-1}$, $F(v):=f(v, v) /\|f(v, v)\|$. It suffices to show that $F$ has a fixed point. Since any fixed point-free map from $S^{n-1}$ to $S^{n-1}$ has degree equal to $(-1)^{n}$, it is enough to show that $F$ has even degree. If $F$ is not surjective, then it is homotopic to a constant map and so has degree 0 , and we are done. When $F$ is surjective, Sard's Theorem gives the existence of a regular value $w$ of $F$. At each point $v$ of $F^{-1}(w)$ the differential of $F$ has full rank, so $F$ is a diffeomorphism in a neighbourhood of $v$. This implies that $F^{-1}(w)$ is discrete in $S^{n-1}$ and so finite. But then the local degree $\operatorname{deg}(F \mid v)$ of $F$ at $v$ is 1 or -1 and we conclude that $\operatorname{deg}(F)=\sum_{v \in F^{-1}(w)} \operatorname{deg}(F \mid v) \equiv\left|F^{-1}(w)\right| \equiv 0 \bmod 2$, since $v \in F^{-1}(w)$ gives $-v \in F^{-1}(w)$ too.

So there is some unit vector $v_{1} \in V$ with $f\left(v_{1}, v_{1}\right) \in \operatorname{span}\left(v_{1}\right)$. Then take the orthogonal complement $W$ of $\operatorname{span}\left(v_{1}\right)$ in $V$. Applying the induction hypothesis to the map $h:=\left.\pi_{W} \circ f\right|_{S^{2} W}: S^{2} W \rightarrow W$, where $\pi_{W}: V \rightarrow W$ is orthogonal projection onto $W$, yields an orthonormal basis $v_{2}, \ldots, v_{n}$ with $h\left(v_{i}, v_{i}\right) \in \operatorname{span}\left(v_{2}, \ldots, v_{i}\right)$. This gives $f\left(v_{i}, v_{i}\right) \in \operatorname{span}\left(v_{1}, \ldots, v_{i}\right)$ for all $i=2, \ldots, n$, and the result follows. 
Looking at (3.6), one may imagine that the following lemma will be useful in proving that all $K_{X}$ are complex diagonalizable:

Lemma 3.7 If $P \in \mathbb{C}^{n \times n}$ has $G(P)=P^{2}+P^{*} P+a P$ skew-Hermitian for some $a \in \mathbb{R}$, then $P$ is diagonalisable via a unitary transformation and the real parts $\mu$ of the eigenvalues of $P$ satisfy $\mu(2 \mu+a)=0$.

Proof Write $P=H+S$, where $H=\left(P+P^{*}\right) / 2$ is Hermitian, $S=\left(P-P^{*}\right) / 2$ is skew-Hermitian. Then $G(P)=2 H^{2}+(H S+S H)+(H S-S H)+a H+a S$. Noting that $H S+S H$ is skew-Hermitian and $[H, S]=H S-S H$ is Hermitian, we see that the Hermitian part of $G(P)$ is $2 H^{2}+a H+[H, S]$. By applying a unitary transformation, we may assume that $H$ is diagonal, i.e. $H=\operatorname{diag}\left(\mu_{1}, \ldots, \mu_{n}\right)$ for certain $\mu_{1}, \ldots, \mu_{n} \in \mathbb{R}$.

Let now $G(P)$ be skew-Hermitian. Then $0=2 H^{2}+a H+[H, S]$. But $2 H^{2}+a H=$ $\operatorname{diag}\left(\mu_{1}\left(2 \mu_{1}+a\right), \ldots, \mu_{n}\left(2 \mu_{n}+a\right)\right)$ and $[H, S]$ has no diagonal component. Hence, both terms have to vanish individually. In particular, $S$ commutes with $H$ and so preserves the eigenspaces of $H$. But $S$ is also diagonalisable, with eigenvalues in $i \mathbb{R}$, and the result follows.

From this, we get

Proposition 3.8 Let $(\mathfrak{a}, \omega)$ be two-step shear data such that the shear is SKT. Then there is a unitary basis $\left(Y_{1}, \ldots, Y_{m}\right)$ of $\mathfrak{a}_{J}$ and $\alpha_{1}, \ldots, \alpha_{m} \in \mathfrak{a}_{r}^{*} \otimes \mathbb{C}$ such that

$$
K_{X}\left(Y_{i}\right)=\alpha_{i}(X) \cdot Y_{i}
$$

for all $X \in \mathfrak{a}_{r}$.

Proof Let $m:=\operatorname{dim}\left(\mathfrak{a}_{r}\right)$. By Lemma 3.6 and Lemma 3.3(iii), there is a basis $\left(X_{1}, \ldots, X_{m}\right)$ of $\mathfrak{a}_{r}$ with $f\left(X_{i}, X_{i}\right) \in \operatorname{span}\left(X_{1}, \ldots, X_{i}\right)$. Set $K_{i}:=K_{X_{i}}$ for $i=1, \ldots, m$ and note that $K_{i}$ is a complex endomorphism of the complex vector space $\left(\mathfrak{a}_{J}, J\right)$ by Lemma 3.3(ii). We need to show that $K_{1}, \ldots, K_{m}$ are simultaneously complex diagonalizable. For this, note that these endomorphisms commute by (3.5) in Lemma 3.5. We show inductively that $K_{1}, \ldots, K_{m}$ are simultaneously complex diagonalizable for all $m=1, \ldots, n$.

For $m=1$, this follows directly from (3.6) in Lemmas 3.5, 3.7 and $f\left(X_{1}, X_{1}\right) \in \operatorname{span}\left(X_{1}\right)$.

Now let us assume that we have shown that $K_{1}, \ldots, K_{m}$ are simultaneously complex diagonalizable for some $s \in\{1, \ldots, m-1\}$. As $K_{m+1}$ commutes with $K_{1}, \ldots, K_{s}$, it preserves the common complex eigenspaces of $K_{1}, \ldots, K_{s}$ and so we may restrict to such an eigenspace, i.e. we may assume that $K_{i}=\lambda_{i} \mathrm{id}_{\mathfrak{a}_{J}}$ for some $\lambda_{i} \in \mathbb{C}, i=1, \ldots, s$. Decompose now $K_{i}=H_{i}+S_{i}$ into its Hermitian part $H_{i}$ and skew-Hermitian part $S_{i}$ for $i=1, \ldots, m$. We may find a unitary basis $\left(Y_{1}, \ldots, Y_{k}\right)$ of $\left(\mathfrak{a}_{J}, g, J\right), k:=\operatorname{dim}_{\mathbb{C}}\left(\mathfrak{a}_{J}\right)$ such that $H_{s+1}=\operatorname{diag}\left(\mu_{1}, \ldots, \mu_{k}\right)$ for certain $\mu_{1}, \ldots, \mu_{k} \in \mathbb{R}$ with respect to that basis. By (3.6) in Lemma 3.5, the Hermitian part of of $K_{s+1}^{*} K_{s+1}+K_{s+1} K_{s+1}+\sum_{i=1}^{s+1} a_{i} K_{i}$ is zero, where $a_{1}, \ldots, a_{s+1} \in \mathbb{R}$ are such that $f\left(X_{s+1}, X_{s+1}\right)=\sum_{i=1}^{s+1} a_{i} X_{i}$. This Hermitian part is given by $2 H_{s+1}^{2}+\left[H_{s+1}, S_{s+1}\right]+\sum_{i=1}^{s} a_{1} \operatorname{Re}\left(\lambda_{i}\right) \operatorname{id}_{\mathfrak{a}_{J}}+a_{s+1} H_{s+1}$. Since all terms are diagonal in the unitary basis $\left(Y_{1}, \ldots, Y_{k}\right)$, apart from $\left[H_{s+1}, S_{s+1}\right]$, which is off-diagonal, we get $\left[H_{s+1}, S_{s+1}\right]=0$. But $S_{s+1}$ is complex diagonalizable and so $H_{s+1}$ and $S_{s+1}$ are simultaneously complex diagonalizable. Hence, $K_{s+1}=H_{s+1}+S_{s+1}$ is complex diagonalizable as well, which shows the statement for $s+1$ and so by induction also for $s=m$.

It is now tempting to assume that certain parts of these decompositions vanish and this is what we will now do. 


\section{Two-step SKT shears with totally real complement}

In this case, $U_{J}=0, \omega_{0}$ is fully given by (3.3) and $\omega_{1}=\left(\omega_{1}\right)_{r r}^{J}+\left(\omega_{1}\right)_{r r}^{r}$. Now by Lemma 3.3 (iv) and (iv), we have $\left(\omega_{1}\right)_{r r}^{r}=0$ and $\left(\omega_{1}\right)_{r r}^{J}=-2 J \circ \mathcal{A}(h)$. So here everything is already determined by $A$ as in (3.3). If we decompose $h=h_{1}+h_{2}$ with $h_{1} \in S^{2} \mathfrak{a}_{r}^{*} \otimes \mathfrak{a}_{J}$, $h_{2} \in \Lambda^{2} \mathfrak{a}_{r}^{*} \otimes \mathfrak{a}_{J}$, then $J^{*}\left(\omega_{1}\right)_{r r}^{J}=-2 i h_{2}$.

Next, looking at the results in Lemma 3.3 and in Proposition 3.8 and using a complex basis $Y_{1}, \ldots, Y_{m}$ of $\mathfrak{a}_{J}$ as in Proposition 3.8, (3.5) may be rewritten as $(f(\cdot, \cdot), \cdot) \in S^{3} \mathfrak{a}_{r}^{*} \otimes \mathfrak{a}_{r}$ and

$$
\alpha^{i}\left(X_{1}\right) h^{i}\left(X_{2}, X_{3}\right)-\alpha^{i}\left(X_{2}\right) h^{i}\left(X_{1}, X_{3}\right)=h^{i}\left(X_{2}, f\left(X_{1}, X_{3}\right)\right)-h^{i}\left(X_{1}, f\left(X_{2}, X_{3}\right)\right)
$$

for all $X_{1}, X_{2}, X_{3} \in \mathfrak{a}_{r}$, where $h=\sum_{i=1}^{r} h^{i} Y_{i}$ with $h^{1}, \ldots, h^{m} \in \mathfrak{a}_{r}^{*} \otimes \mathfrak{a}_{r}^{*} \otimes \mathbb{C}$. Imposing these conditions, (2.1) is valid (i.e. our shear defines a Lie algebra) if and only if $\sum_{j=1}^{3} \omega\left(J X_{j}, \omega\left(J X_{j+1}, J X_{j+2}\right)\right)=0$ for all $X_{1}, X_{2}, X_{3} \in \mathfrak{a}_{r}$, which is easily seen to be equivalent to

$$
\alpha_{i} \wedge h_{2}^{i}=0
$$

for all $i=1, \ldots, m$, where we have written $h_{2}=\sum_{i=1}^{m} h_{2}^{i} Y_{i}$ with $h_{2}^{i} \in \Lambda^{2} \mathfrak{a}_{r}^{*} \otimes \mathbb{C}$, $i=1, \ldots, m$. Note that the latter condition is equivalent to $\alpha_{i}=0$ or $h_{2}^{i}=\alpha_{i} \wedge \beta_{i}$ for some $\beta_{i} \in \mathfrak{a}_{r}^{*} \otimes \mathbb{C}$. Summarizing, we need to satisfy the following equations for all $i=1, \ldots, m$ and all $X_{1}, X_{2}, X_{3} \in \mathfrak{a}_{r}$ :

$$
\begin{aligned}
\alpha_{i}\left(X_{1}\right) h^{i}\left(X_{2}, X_{3}\right)-\alpha_{i}\left(X_{2}\right) h^{i}\left(X_{1}, X_{2}\right) & =h^{i}\left(X_{2}, f\left(X_{1}, X_{3}\right)\right)-h^{i}\left(X_{1}, f\left(X_{2}, X_{3}\right)\right), \\
\alpha_{i} \wedge h_{2}^{i} & =0
\end{aligned}
$$

Summarizing, all two-step solvable Lie algebras which are shears of the Abelian Lie algebra with $U_{J}=0$, i.e. which fulfil $\mathfrak{g}=\mathfrak{g}^{\prime}+J \mathfrak{g}^{\prime}$ if we assume that $\omega$ is surjective, are of the following form:

Definition 4.1 Let $\mathfrak{a}_{J}$ be a $m$-dimensional complex vector space with basis $Y_{1}, \ldots, Y_{m}, \mathfrak{a}_{r}$ be a $k$-dimensional real vector space. Moreover, set $U_{r}:=\mathfrak{a}_{r}$ and let $I_{0}: \mathfrak{a}_{r} \rightarrow U_{r}$ be a linear isomorphism. Next, let $f \in S^{2} \mathfrak{a}_{r}^{*} \otimes \mathfrak{a}_{r}, h_{1} \in S^{2} \mathfrak{a}_{r}^{*} \otimes \mathfrak{a}_{J}, h_{2} \in \Lambda^{2} \mathfrak{a}_{r}^{*} \otimes \mathfrak{a}_{J}$ and $\alpha_{1}, \ldots, \alpha_{m} \in \mathfrak{a}_{r}^{*} \otimes \mathbb{C}$ be such that $f(f(\cdot, \cdot), \cdot) \in S^{3} \mathfrak{a}_{r}^{*} \otimes \mathfrak{a}_{r}$ and let $g_{0} \in S^{2} \mathfrak{a}_{r}^{*}$ and $\tau_{0} \in \Lambda^{2} \mathfrak{a}_{r}^{*}$ be given. Set $h:=h_{1}+h_{2}$ and define $h_{1}^{i}$ and $h_{2}^{i}$ for $i=1, \ldots, m$ as above.

Then put

$$
\mathfrak{g}:=\mathfrak{a}_{J} \oplus \mathfrak{a}_{r} \oplus U_{r}
$$

as vector spaces and endow it with the anti-symmetric bilinear map $[\cdot, \cdot]$ uniquely defined by

$$
\begin{aligned}
{\left[Y_{i}, Y_{j}\right] } & =0, \quad\left[X, Y_{i}\right]=0, \quad\left[I_{0} X, Y_{i}\right]=\alpha_{i}(X) \cdot Y_{i}, \\
{\left[I_{0} X, W\right] } & =f(X, W)+h(X, W), \quad\left[I_{0} X, I_{0} W\right]=-2 i h_{2}(X, W)
\end{aligned}
$$

for $i, j \in\{1, \ldots, m\}, X, W \in \mathfrak{a}_{r}$, extended complex linearly if one of the arguments is in $\mathfrak{a}_{J}$. Next, define an almost complex structure $I$ on $\mathfrak{g}$ by

$$
I\left(Y_{j}+X+I_{0} Z\right):=i Y_{j}-Z+I_{0} X
$$


for all $j \in\{1, \ldots, m\}$ and all $X, Z \in \mathfrak{a}_{r}$ and a Euclidean metric $\tilde{g}$ on $\mathfrak{g}$ such that $Y_{1}, \ldots, Y_{m}, i Y_{1}, \ldots, i Y_{m}$ is a $\tilde{g}$-orthonormal basis of (the realification of) $\mathfrak{a}_{J}, \mathfrak{a}_{r} \oplus U_{r}$ is $\tilde{g}$-orthogonal to $\mathfrak{a}_{J}$ and

$$
\tilde{g}(X, W)=\tilde{g}(J X, J W)=g_{0}(X, W), \tilde{g}(J X, W)=-\tilde{g}(X, J W)=\tau_{0}(X, W)
$$

for all $X, W \in \mathfrak{a}_{r}$.

We write $\mathfrak{g}_{f, h, \alpha}$ for the triple $(\mathfrak{g}, \tilde{g}, I)$ or only for $\mathfrak{g}$, where $\alpha:=\left(\alpha_{1}, \ldots, \alpha_{m}\right)$ and we suppress the dependence on the other tensors.

The discussions above and in the last subsection give us:

Proposition 4.2 Let $\mathfrak{g}_{f, h, \alpha}$ be as in Definition 4.3. If the defining data satisfies (4.1), then $\mathfrak{g}_{f, h, \alpha}$ is an almost Hermitian Lie algebra with integrable almost complex structure. Moreover, any two-step solvable SKT Lie algebra $(\mathfrak{g}, g, J)$ with $\mathfrak{g}^{\prime}+J \mathfrak{g}^{\prime}=\mathfrak{g}$ is isomorphic to some $\mathfrak{g}_{f, h, \alpha}$.

Actually, we can say a tiny bit more which will help us classifying all two-step solvable SKT Lie algebras $(\mathfrak{g}, g, J)$ with $\mathfrak{g}^{\prime}+J \mathfrak{g}^{\prime}=\mathfrak{g}$ and $\operatorname{dim}\left(\mathfrak{g}_{r}^{\prime}\right) \leq 2$.

For this, recall that the proof of Lemma 3.6 yields some $v_{1} \in V \backslash\{0\}$ such that either $f\left(v_{1}, v_{1}\right)=0$ or $f\left(v_{1}, v_{1}\right)=v_{1}$. In fact, the latter case is always true if we additionally have that $\operatorname{im}(f)=V$ and $f(f(\cdot, \cdot), \cdot) \in S^{3} V^{*} \otimes V$. Even more, $f\left(v_{1}, \cdot\right)=\operatorname{id}_{V}$ :

Proposition 4.3 Let $V$ be an n-dimensional real vector space and $f \in S^{2} V^{*} \otimes V$ be such that $f(f(\cdot, \cdot), \cdot) \in S^{3} V^{*} \otimes V$ and $\operatorname{im}(f)=V$. Then there exists $a v \in V$ with $f(v, \cdot)=\operatorname{id}_{V}$.

Proof The symmetry assumptions on $f$ imply that the endomorphisms $f_{v}=f(v, \cdot), v \in V$, commute and form a closed algebra: $f_{u} \circ f_{v}=f_{f(u, v)}$. It is enough to show that $\mathrm{id}_{V}$ is in the algebra $A$ which is the complex linear span of $\left\{f_{v}: v \in V\right\}$, since $\operatorname{id}_{V}=\sum_{i=1}^{k} a_{i} f_{v_{i}}$ with $a_{i} \in \mathbb{C}$ implies id $\operatorname{id}_{V}=\sum_{i=1}^{k} \operatorname{Re}\left(a_{i}\right) f_{v_{i}}$, as $f_{v_{i}}$ are real. Extending $f$ complex linearly, it is enough to prove the assertion on the complexification $V \otimes \mathbb{C}$.

Now the operators $f_{v}, v \in V \otimes \mathbb{C}$, commute, so $V \otimes \mathbb{C}$ is a direct sum of subspaces $W$, on which each $f_{v}$ has only a single eigenvalue. The assumption that $f$ is surjective on the real space $V$, implies that it is also surjective on $V \otimes \mathbb{C}$. So for a given summand $W$ there is $u(w) \in V \otimes \mathbb{C}$ for which $f_{u(w)}$ has a non-zero eigenvalue $\lambda$. It follows there is a linear combination $a$ of these $f_{u(w)}$ that has all eigenvalues non-zero. Now consider the characteristic polynomial $\Delta_{a}(t)=\operatorname{det}\left(a-t \mathrm{id}_{V}\right)=c+p_{a}(t)$, where $c=\operatorname{det}(a) \neq 0$ and $p_{a}(t)$ is a polynomial in $t$ without constant term. By the Cayley-Hamilton theorem, $0=\Delta_{a}(a)=c \operatorname{id}_{V}+p_{a}(a)$, so id $V=-p_{a}(a) / c$ which is an element of the algebra $A$ and hence has the form $f_{w}$ for some $w \in V \otimes \mathbb{C}$. By the remarks above we have $\operatorname{id}_{V}=f_{v}$ for $v=\operatorname{Re}(w)$, as required.

Proposition 4.3 yields:

Corollary 4.4 Let $(\mathfrak{g}, g, J)$ be a two-step solvable Lie algebra with $\mathfrak{g}^{\prime}+J \mathfrak{g}^{\prime}=\mathfrak{g}$. Then there exist some $X \in \mathfrak{g}_{r}^{\prime}$ and $h_{1}, h_{2} \in \operatorname{End}\left(\mathfrak{g}_{r}^{\prime}, \mathfrak{g}_{J}^{\prime}\right)$ such that for all $W \in \mathfrak{g}_{r}^{\prime}$ we have

$$
[J X, W]=W+h_{1}(W), \quad[J W, X]=W+h_{2}(W) .
$$

In particular, $\mathfrak{g}$ is not nilpotent.

Note that Proposition 4.3 does not give us any information on the endomorphism $f(w, \cdot)$ for $w \notin \operatorname{span}(v)$ besides $f(w, v)=w$. This is one of the reasons why we are restricting in the following to the case $\operatorname{dim}\left(\mathfrak{g}_{r}^{\prime}\right) \leq 2$. Another reason is that surely (4.1) gets easier in this case, in particular, the second equation in (4.1) is void in this case. 


\subsection{Almost Abelian algebras}

An almost Abelian Lie algebra is one with a codimension one Abelian ideal and the SKT structures on such Lie algebras of dimension $2 n$ are precisely those which may be obtained by a shear from the flat Kähler SKT Lie algebra $\left(\mathbb{R}^{2 n}, g_{0}, J_{0}, \sigma_{0}\right)$ with $\operatorname{dim}(\mathfrak{a})=2 n-1$ or, equivalently, with $U_{J}=0$ and $\operatorname{dim}\left(\mathfrak{a}_{r}\right)=1$. Note that a characterization of those almost Abelian Lie algebras which are SKT has already been given in [4], cf. also [10] for a more detailed list in the six-dimensional case.

We prove this characterization by a different approach and even solve the remaining equations in [4] completely. Note further that, in contrast to all other cases in this paper, we will not assume here that $\operatorname{im}(\omega)=\mathfrak{a}$, i.e. we also do not necessarily have $\mathfrak{g}^{\prime}+J \mathfrak{g}^{\prime}=\mathfrak{g}$. Still, one easily sees that we may use all the results from above apart from Corollary 4.4, where we use the mentioned identity in the proof.

Choose $X \in \mathfrak{a}_{r}$ of norm one. Then $f(X, X)=a X$ for some $a \in \mathbb{R}$ and $h(X, X)=$ $\sum_{i=1}^{n-1} w_{i} Y_{i}$ for certain $w_{1}, \ldots, w_{n-1} \in \mathbb{C}$. Moreover, $\alpha_{i}(X)=z_{i}$ for $z_{i} \in \mathbb{C}, i=1, \ldots, n-$ 1. Note that here also the first equation in (4.1) is void in total and so we only still need to impose (3.2), i.e. $v(\omega)=0$. But by Lemma 3.4 and for dimensional reasons, $v(\omega)$ only has a component in $\Lambda^{2} \mathfrak{a}_{J}^{*} \wedge \mathfrak{a}_{r}^{*} \wedge U_{r}^{*}$. The corresponding equation is given in (3.6) and is equivalent to

$$
\bar{z}_{i} z_{i}+z_{i}^{2}+a z_{i}=-\overline{\bar{z}_{i} z_{i}+z_{i}^{2}+a z_{i}}
$$

This may be written as $\operatorname{Re}\left(z_{i}\right)\left(2 \operatorname{Re}\left(z_{i}\right)+a\right)=0$. So $\operatorname{Re}\left(z_{i}\right) \in\{0,-a / 2\}$ for any $i=$ $1, \ldots, n-1$. Thus, we have obtained:

Theorem 4.5 Let $(\mathfrak{g}, g, J)$ be a $2 n$-dimensional almost Abelian SKT Lie algebra. Then $(\mathfrak{g}, g, J)$ is isomorphic to $\mathfrak{g}_{f, h, \alpha}$ with $\mathfrak{a}_{r}=\operatorname{span}(X)$ for a unit vector $X, h(X, X)=$ $\sum_{i=1}^{n-1} w_{i} Y_{i}, f(X, X)=a X$ and $\alpha(X)=\sum_{i=1}^{n-1} z_{i} Y_{i}$ for certain $a \in \mathbb{R}, z=\left(z_{1}, \ldots, z_{n-1}\right)$, $w=\left(w_{1}, \ldots, w_{n-1}\right) \in \mathbb{C}^{n-1}$ with $\operatorname{Re}\left(z_{i}\right) \in\{0,-a / 2\}$ for $i=1, \ldots, n-1$. Conversely, all such $\mathfrak{g}_{f, h, \alpha}$ are almost Abelian SKT Lie algebras.

Remark 4.6 So any almost Abelian SKT Lie algebra $(\mathfrak{g}, g, J)$ with codimension one Abelian ideal $\mathfrak{a}$ has a real basis $Y_{1}, \ldots, Y_{n-1}, i Y_{1}, \ldots, i Y_{n-1}, X, J X$, where $Y_{1}, \ldots, Y_{n-1}$ is a complex basis of $\mathfrak{a}_{J}$ and $X$ spans $\mathfrak{a}_{r}$, such that $Y_{1}, \ldots, Y_{n-1}, i Y_{1}, \ldots, i Y_{n-1}, X$ is an orthonormal system and there is some $m \in\{0, \ldots, n-1\}$ so the only non-zero Lie brackets (up to antisymmetry and complex-linear extension of the bracket to $\mathfrak{a}_{J}$ ) are given by

$$
\left[J X, Y_{j}\right]=\left(-\frac{a}{2}+i b_{j}\right) Y_{j}, \quad\left[J X, Y_{k}\right]=i b_{k} Y_{k}, \quad[J X, X]=a X+\sum_{i=1}^{m} w_{i} Y_{i}
$$

for $j=1, \ldots, m, k=m+1, \ldots, n-1$ and certain $a \in \mathbb{R}, b=\left(b_{1}, \ldots, b_{n}\right) \in \mathbb{R}^{n-1}$, $w=\left(w_{1}, \ldots, w_{n-1}\right) \in \mathbb{C}^{n-1}$. For definiteness, we take $a \neq 0$ if $m>0$.

Observe that here the unimodular cases are exactly those with $m=1$ or $a=0$. Note that $\mathfrak{g}^{\prime} \oplus J \mathfrak{g}^{\prime}=\mathfrak{g}$ holds if $a \neq 0$ and $b_{j} \neq 0$ for all $j=m+1, \ldots, n-1$.

If $\mathfrak{g}^{\prime} \oplus J \mathfrak{g}^{\prime} \neq \mathfrak{g}$, then, in contrast to Corollary 4.4, $\mathfrak{g}$ may be nilpotent. However, this is only possible if $m=n-1, a=0$ and $b_{j}=0$ for all $j=1, \ldots, n$. In that case, $\mathfrak{g}$ is isomorphic to $\mathfrak{h}_{3} \oplus \mathbb{R}^{2 n-3}$.

Since two almost Abelian Lie algebras $\mathbb{R}^{2 n-1} \rtimes_{f_{1}} \mathbb{R}$ and $\mathbb{R}^{2 n-1} \rtimes_{f_{2}} \mathbb{R}$ are isomorphic if and only if $f_{1}$ is conjugated to $f_{2}$ up to a non-zero multiple, Theorem 4.5 implies 
Corollary 4.7 A $2 n$-dimensional almost Abelian Lie algebra $\mathbb{R}^{2 n-1} \rtimes_{f} \mathbb{R}$ admits an SKT structure if and only if

(i) either $f$ is diagonalizable over the complex numbers and there is one real eigenvalue $a \in \mathbb{R}$ such that the other complex eigenvalues come in $n-1$ pairs $\left(z_{i}, \bar{z}_{i}\right)$ with $\operatorname{Re}\left(z_{i}\right) \in\{0,-a / 2\}, i=1, \ldots, n-1$,

(ii) or the complex Jordan normal form of $f$ has one Jordan block of size 2 with eigenvalue 0 and all other Jordan block are of size 1 with eigenvalues $i b_{1}$, $-i b_{1}, \ldots, i b_{n-2},-i b_{n-2}, 0$ for certain real numbers $b_{1}, \ldots, b_{n-2} \in \mathbb{R}$.

In the six-dimensional case, Corollary 4.7 yields, in agreement with [10], that the following almost Abelian Lie algebras admit an SKT-structure, where we refer to Table 1 for the Lie brackets of the mentioned Lie algebras:

Corollary 4.8 Let $\mathfrak{g}$ be a six-dimensional almost Abelian Lie algebra. Then $\mathfrak{g}$ admits an SKT structure if and only if $\mathfrak{g}$ is isomorphic to one of the following Lie algebras:

$$
\begin{aligned}
& \mathfrak{g}_{6,1}^{-\frac{1}{2},-\frac{1}{2},-\frac{1}{2},-\frac{1}{2}}, \mathfrak{g}_{6,8}^{a,-\frac{a}{2},-\frac{a}{2},-\frac{a}{2}}, \mathfrak{g}_{6,8}^{a,-\frac{a}{2},-\frac{a}{2}, 0}, \underset{\mathfrak{g}_{6,11}^{a,-\frac{a}{2},-\frac{a}{2}, s}, \mathfrak{g}_{6,11}^{a, 0,-\frac{a}{2}, s},}{\quad} \\
& \mathfrak{g}_{6,11}^{a,-\frac{a}{2}, 0, s}, \mathfrak{g}_{6,11}^{a, 0,0, s}, \mathfrak{g}_{5,14}^{0} \oplus \mathbb{R}, \mathfrak{g}_{5,17}^{0,0, s} \oplus \mathbb{R}, \mathfrak{r}_{4,-1 / 2,-1 / 2} \oplus \mathbb{R}^{2}, \\
& \mathfrak{r}_{4, a,-\frac{a}{2}}^{\prime} \oplus \mathbb{R}^{2}(a>0), \mathfrak{r}_{4, a, 0}^{\prime} \oplus \mathbb{R}^{2}(a>0), \mathfrak{r}_{3,0}^{\prime} \oplus \mathbb{R}^{3}, \mathfrak{h}_{3} \oplus \mathbb{R}^{3}, \mathfrak{a f f} \mathfrak{R}_{\mathbb{R}} \oplus \mathbb{R}^{4}, \mathbb{R}^{6} .
\end{aligned}
$$

\subsection{Commutator ideal not complex and of codimension two}

Note that if $\mathfrak{g}^{\prime}$ is of codimension two, the condition $\mathfrak{g}^{\prime}+J \mathfrak{g}^{\prime}=\mathfrak{g}$ is equivalent to $\mathfrak{g}^{\prime}$ being non-complex.

By Proposition 4.3, we may choose an orthonormal basis $X_{1}, X_{2}$ of $\mathfrak{a}_{r}$ such that $f\left(X_{1}, \cdot\right)=a \operatorname{id}_{\mathfrak{a}_{r}}$ for some $a>0$ and such that $f\left(X_{2}, X_{2}\right)=b_{1} X_{1}+b_{2} X_{2}$ for certain $b_{1}, b_{2} \in \mathbb{R}$ with $b_{2} \geq 0$.

We set $z_{i}:=\alpha_{i}\left(X_{1}\right), w_{i}:=\alpha_{i}\left(X_{2}\right) \in \mathbb{C}$ for $i=1, \ldots, n-2$ and first look at (3.6). As in the last subsection, this equation, applied to $X=W=X_{1}$, gives us $\operatorname{Re}\left(z_{i}\right) \in\{0,-a / 2\}$. Next, applying (3.6) to $X=X_{1}, W=X_{2}$, we obtain

$$
\operatorname{Re}\left(w_{i}\right)\left(2 \operatorname{Re}\left(z_{i}\right)+a\right)=0,
$$

so that $\operatorname{Re}\left(w_{i}\right)=0$ if $\operatorname{Re}\left(z_{i}\right)=0$. Otherwise, the above equation is automatically fulfilled. Finally, if $X=W=X_{2}$, (3.6) yields

$$
2 \operatorname{Re}\left(w_{i}\right)^{2}+b_{2} \operatorname{Re}\left(w_{i}\right)+b_{1} \operatorname{Re}\left(z_{i}\right)=0 .
$$

If $\operatorname{Re}\left(z_{i}\right)=0$ and so also $\operatorname{Re}\left(z_{i}\right)=0$, this equation is automatically satisfied. Otherwise, $\operatorname{Re}\left(w_{i}\right)$ has to be a solution of the quadratic equation

$$
2 x^{2}+b_{2} x-\frac{1}{2} b_{1} a=0,
$$

which has a solution if $b_{2}^{2}+4 b_{1} a \geq 0$. So our parameters $a, b_{1}, b_{2}$ have to fulfil this inequality if for some $i \in\{1, \ldots, n-2\}$ we have $\operatorname{Re}\left(z_{i}\right) \neq 0$.

Next, we look at the first equation in (4.1), which yields

$$
\begin{aligned}
& z_{i} h^{i}\left(X_{2}, X_{1}\right)-w_{i} h^{i}\left(X_{1}, X_{1}\right)=a h^{i}\left(X_{2}, X_{1}\right)-a h^{i}\left(X_{1}, X_{2}\right), \\
& z_{i} h^{i}\left(X_{2}, X_{2}\right)-w_{i} h^{i}\left(X_{1}, X_{2}\right)=a h^{i}\left(X_{2}, X_{2}\right)-b_{1} h^{i}\left(X_{1}, X_{1}\right)-b_{2} h^{i}\left(X_{1}, X_{2}\right) .
\end{aligned}
$$


Since $z_{i}-a \neq 0$ due to $\operatorname{Re}\left(z_{i}\right) \in\{0,-a / 2\}$ and $a \neq 0$, these equations are equivalent to

$$
\begin{aligned}
& h^{i}\left(X_{2}, X_{1}\right)=\frac{w_{i}}{z_{i}-a} h^{i}\left(X_{1}, X_{1}\right)-\frac{a}{z_{i}-a} h^{i}\left(X_{1}, X_{2}\right), \\
& h^{i}\left(X_{2}, X_{2}\right)=-\frac{b_{1}}{z_{i}-a} h^{i}\left(X_{1}, X_{1}\right)+\frac{w_{i}-b_{2}}{z_{i}-a} h^{i}\left(X_{1}, X_{2}\right) .
\end{aligned}
$$

Summarizing the discussion up to this point, we have obtained:

Lemma 4.9 Let $(\mathfrak{g}, g, J)$ be a two-step solvable SKT Lie algebra with $\mathfrak{g}^{\prime}$ being non-complex and of codimension two. Then $(\mathfrak{g}, g, J)$ is isomorphic to $\mathfrak{g}_{f, h, \alpha}$ with $X_{1}, X_{2}$ being an orthonormal basis of $\mathfrak{a}_{r}, f$ satisfying

$$
f\left(X_{1}, X_{1}\right)=a X_{1}, \quad f\left(X_{1}, X_{2}\right)=f\left(X_{2}, X_{1}\right)=a X_{2}, \quad f\left(X_{2}, X_{2}\right)=b_{1} X_{1}+b_{2} X_{2}
$$

for certain $a>0, b_{2} \geq 0, h$ fulfilling (4.7) and for all $i=1, \ldots, n-2$, and $z_{i}, w_{i} \in \mathbb{C}$ defined by

$$
\alpha_{i}\left(X_{1}\right)=z_{i}, \quad \alpha\left(X_{2}\right)=w_{i}
$$

satisfy either

(i) $\operatorname{Re}\left(z_{i}\right)=\operatorname{Re}\left(w_{i}\right)=0$, or

(ii) $\operatorname{Re}\left(z_{i}\right)=-a / 2$ and $2 \operatorname{Re}\left(w_{i}\right)^{2}+b_{2} \operatorname{Re}\left(w_{i}\right)-\frac{1}{2} b_{1} a=0$, where this case may only occur if $b_{1} \geq-b_{2}^{2} /(4 a)$.

Moreover, with this data, $\mathfrak{g}_{f, h, \alpha}$ is an almost Hermitian Lie algebra with integrable almost complex structure I.

We still need to solve (3.2) and remark that we already solved it on $\Lambda^{2} \mathfrak{a}_{J} \wedge \mathfrak{a}_{r} \wedge U_{r}$. Using Lemma 3.4 and for dimensional reasons, we only have to consider this equation on $\Lambda^{2} \mathfrak{a}_{r} \wedge \Lambda^{2} U_{r}, \mathfrak{a}_{J} \wedge \Lambda^{2} \mathfrak{a}_{r} \wedge U_{r}$ and $\mathfrak{a}_{J} \wedge \mathfrak{a}_{r} \wedge \Lambda^{2} U_{r}$. We first look at the latter two equations. Let $Y \in \mathfrak{a}_{J}$ and $k \in\{1,2\}$ be given. Since $J^{*} v_{1}(\omega)=v_{1}(\omega)$, we have $v_{1}(\omega)\left(Y, X_{1}, X_{2}, J X_{k}\right)=-v_{1}(\omega)\left(i Y, J X_{1}, J X_{2}, X_{k}\right)$ and so $v(\omega)\left(Y, X_{1}, X_{2}, J X_{k}\right)=0$ and $v(\omega)\left(i Y, X_{k}, J X_{1}, J X_{2}\right)=0$ are equivalent to

$$
\begin{aligned}
v_{1}\left(Y, X_{1}, X_{2}, J X_{k}\right)+2 v_{2}\left(Y, X_{1}, X_{2}, J X_{k}\right) & =0, \\
v_{2}\left(Y, X_{1}, X_{2}, J X_{k}\right) & =-v_{2}\left(i Y, J X_{1}, J X_{2}, X_{k}\right) .
\end{aligned}
$$

In the following, we use that $\omega(\mathfrak{a}, \mathfrak{a})=0, \omega\left(U_{r}, \mathfrak{a}_{J}\right) \subseteq \mathfrak{a}_{J}, \omega\left(U_{r}, U_{r}\right) \subseteq \mathfrak{a}_{J}, \mathfrak{a}_{J} \perp \mathfrak{a}_{r}$ and the fact that $f$ is symmetric and $f(f(\cdot, \cdot), \cdot) \in S^{3} \mathfrak{a}_{r}^{*} \otimes \mathfrak{a}_{r}$ to simplify these equations and first look at the second equation. We have

$$
\begin{aligned}
v_{1}(\omega)\left(X_{1}, X_{2}, J X_{k}, Y\right)= & \frac{1}{12} g\left(-h\left(f\left(X_{k}, X_{1}\right), X_{2}\right)+h\left(X_{2}, f\left(X_{k}, X_{1}\right)\right)\right. \\
& +K_{X_{2}}\left(h\left(X_{k}, X_{1}\right)\right)+h\left(f\left(X_{k}, X_{2}\right), X_{1}\right) \\
& \left.-h\left(X_{1}, f\left(X_{k}, X_{2}\right)\right)-K_{X_{1}}\left(h\left(X_{k}, X_{2}\right)\right), i Y\right)
\end{aligned}
$$

and

$$
\begin{aligned}
- & v_{2}(\omega)\left(J X_{1}, J X_{2}, X_{k}, i Y\right) \\
= & \frac{1}{12} g\left(K_{X_{k}}\left(h\left(X_{2}, X_{1}\right)-h\left(X_{1}, X_{2}\right)\right)\right. \\
& \left.+h\left(f\left(X_{k}, X_{2}\right), X_{1}\right)-h\left(f\left(X_{k}, X_{1}\right), X_{2}\right), i Y\right),
\end{aligned}
$$


i.e. $v_{2}(\omega)\left(Y, X_{1}, X_{2}, J X_{k}\right)=-v_{2}(\omega)\left(i Y, J X_{1}, J X_{2}, X_{k}\right)$ for all $Y \in \mathfrak{a}_{J}$ is equivalent to

$$
\begin{aligned}
- & h\left(f\left(X_{k}, X_{1}\right), X_{2}\right)+h\left(X_{2}, f\left(X_{k}, X_{1}\right)\right)+K_{X_{2}}\left(h\left(X_{k}, X_{1}\right)\right)+h\left(f\left(X_{k}, X_{2}\right), X_{1}\right) \\
& -h\left(X_{1}, f\left(X_{k}, X_{2}\right)\right)-K_{X_{1}}\left(h\left(X_{k}, X_{2}\right)\right) \\
& =K_{X_{k}}\left(h\left(X_{2}, X_{1}\right)-h\left(X_{1}, X_{2}\right)\right)+h\left(f\left(X_{k}, X_{2}\right), X_{1}\right)-h\left(f\left(X_{k}, X_{1}\right), X_{2}\right) .
\end{aligned}
$$

However, by (3.5), we have

$$
\begin{aligned}
- & h\left(f\left(X_{k}, X_{1}\right), X_{2}\right)+h\left(X_{2}, f\left(X_{k}, X_{1}\right)\right)+K_{X_{2}}\left(h\left(X_{k}, X_{1}\right)\right) \\
& +h\left(f\left(X_{k}, X_{2}\right), X_{1}\right)-h\left(X_{1}, f\left(X_{k}, X_{2}\right)\right)-K_{X_{1}}\left(h\left(X_{k}, X_{2}\right)\right) \\
= & -h\left(f\left(X_{k}, X_{1}\right), X_{2}\right)+h\left(X_{k}, f\left(X_{2}, X_{1}\right)\right)+K_{X_{k}}\left(h\left(X_{2}, X_{1}\right)\right) \\
& +h\left(f\left(X_{k}, X_{2}\right), X_{1}\right)-h\left(X_{k}, f\left(X_{1}, X_{2}\right)\right)-K_{X_{k}}\left(h\left(X_{1}, X_{2}\right)\right) \\
= & K_{X_{k}}\left(h\left(X_{2}, X_{1}\right)-h\left(X_{1}, X_{2}\right)\right)+h\left(f\left(X_{k}, X_{2}\right), X_{1}\right)-h\left(f\left(X_{k}, X_{1}\right), X_{2}\right),
\end{aligned}
$$

i.e $v_{2}(\omega)\left(Y, X_{1}, X_{2}, J X_{k}\right)=-v_{2}(\omega)\left(i Y, J X_{1}, J X_{2}, X_{k}\right)$ is automatically satisfied.

Next, we consider $v_{1}\left(Y, X_{1}, X_{2}, J X_{k}\right)+2 v_{2}\left(Y, X_{1}, X_{2}, J X_{k}\right)=0$ and note that

$$
\begin{aligned}
6 v_{1}\left(X_{1}, X_{2}, J X_{k}, Y\right)= & g\left(K_{X_{2}}^{T}\left(h\left(X_{k}, X_{1}\right)\right)-K_{X_{1}}^{T}\left(h\left(X_{k}, X_{2}\right)\right)\right. \\
& \left.+K_{X_{k}}^{T}\left(h\left(X_{2}, X_{1}\right)-h\left(X_{1}, X_{2}\right)\right), i Y\right) .
\end{aligned}
$$

Thus, using that $v_{2}\left(X_{1}, X_{2}, J X_{k}, Y\right)=-v_{2}(\omega)\left(J X_{1}, J X_{2}, X_{k}, i Y\right)$, one sees that $v_{1}\left(X_{1}, X_{2}, J X_{k}, Y\right)+2 v_{2}\left(X_{1}, X_{2}, J X_{k}, Y\right)=0$ for all $Y \in \mathfrak{a}_{J}$ is equivalent to

$$
\begin{aligned}
0= & \left(K_{X_{k}}+K_{X_{k}}^{T}\right)\left(h\left(X_{2}, X_{1}\right)-h\left(X_{1}, X_{2}\right)\right)+h\left(f\left(X_{k}, X_{2}\right), X_{1}\right) \\
& -h\left(f\left(X_{k}, X_{1}\right), X_{2}\right)+K_{X_{2}}^{T}\left(h\left(X_{k}, X_{1}\right)\right)-K_{X_{1}}^{T}\left(h\left(X_{k}, X_{2}\right)\right) .
\end{aligned}
$$

We look at these equations componentwise and for $k=1$, 2. For $k=1$, we obtain

$$
0=\left(2 \operatorname{Re}\left(z_{i}\right)+a\right)\left(h^{i}\left(X_{2}, X_{1}\right)-h^{i}\left(X_{1}, X_{2}\right)\right)-\bar{z}_{i} h^{i}\left(X_{1}, X_{2}\right)+\bar{w}_{i} h^{i}\left(X_{1}, X_{1}\right),
$$

whereas for $k=2$, we get

$$
\begin{aligned}
0= & b_{1} h^{i}\left(X_{1}, X_{1}\right)-2 \operatorname{Re}\left(w_{i}\right) h^{i}\left(X_{1}, X_{2}\right) \\
& +\left(2 \operatorname{Re}\left(w_{i}\right)+b_{2}+\bar{w}_{i}\right) h^{i}\left(X_{2}, X_{1}\right)-\left(\bar{z}_{i}+a\right) h^{i}\left(X_{2}, X_{2}\right) .
\end{aligned}
$$

Now either $\operatorname{Re}\left(z_{i}\right)=0$ or $\operatorname{Re}\left(z_{i}\right)=-a / 2$.

In the first case, (4.9) gives us

$$
\begin{aligned}
h^{i}\left(X_{2}, X_{1}\right) & =-\frac{\bar{w}_{i}}{a} h^{i}\left(X_{1}, X_{1}\right)+\frac{\bar{z}_{i}+a}{a} h^{i}\left(X_{1}, X_{2}\right) \\
& =\frac{w_{i}}{a} h^{i}\left(X_{1}, X_{1}\right)+\frac{a-z_{i}}{a} h^{i}\left(X_{1}, X_{2}\right)
\end{aligned}
$$

Together with (4.7), we obtain

$$
\frac{w_{i}}{z_{i}-a} h^{i}\left(X_{1}, X_{1}\right)-\frac{a}{z_{i}-a} h^{i}\left(X_{1}, X_{2}\right)=\frac{w_{i}}{a} h^{i}\left(X_{1}, X_{1}\right)+\frac{a-z_{i}}{a} h^{i}\left(X_{1}, X_{2}\right)
$$
i.e.

$$
z_{i} h^{i}\left(X_{1}, X_{2}\right)=w_{i} h^{i}\left(X_{1}, X_{1}\right)
$$

where we have used that $2 a-z_{i} \neq 0$. 
So we have to discuss the two subcases $z_{i} \neq 0$ and $z_{i}=0$. First for $z_{i} \neq 0$ : Here we get $h^{i}\left(X_{2}, X_{1}\right)=\frac{w_{i}}{z_{i}} h^{i}\left(X_{1}, X_{1}\right)$ and so, inserting into (4.7), we obtain

$$
h^{i}\left(X_{2}, X_{1}\right)=\frac{w_{i}}{z_{i}} h^{i}\left(X_{1}, X_{1}\right)=h^{i}\left(X_{1}, X_{2}\right)
$$

and

$$
h^{i}\left(X_{2}, X_{2}\right)=\frac{w_{i}^{2}-b_{2} w_{i}-b_{1} z_{i}}{z_{i}\left(z_{i}-a\right)} h^{i}\left(X_{1}, X_{1}\right),
$$

and one checks that (4.9) and (4.10) are also satisfied then.

Second, for $z_{i}=0$ : Then $w_{i} h^{i}\left(X_{1}, X_{1}\right)=0$ and the first equation in (4.7) gives us $h^{i}\left(X_{2}, X_{1}\right)=h^{i}\left(X_{1}, X_{2}\right)$ and (4.9) is automatically satisfied. We now show $w_{i} \neq 0$, because $\operatorname{im}(\omega)=\mathfrak{a}$.

Suppose $w_{i}=0$. Since $\omega\left(J X_{1}, Y_{i}\right)=-z_{i} Y_{i}, \omega\left(J X_{2}, Y_{i}\right)=-w_{i} Y_{i}$ for all $i=1, \ldots, n-$ 2, we may ignore those $i$ where $z_{i} \neq 0$ or $w_{i} \neq 0$, i.e. we may assume that $\omega\left(J X_{i}, \mathfrak{a}_{J}\right)=0$ globally for all $i=1,2$ and $\operatorname{dim}_{\mathbb{R}}\left(\mathfrak{a}_{J}\right) \geq 2$. But then we have $\omega\left(J X_{1}, X_{2}\right)=\omega\left(J X_{2}, X_{1}\right)$ and so $\omega\left(J X_{1}, J X_{2}\right)=0$. Hence, $\omega\left(J X_{1}, X_{1}\right), \omega\left(J X_{1}, X_{2}\right), \omega\left(J X_{2}, X_{2}\right)$ have to span the at least four-dimensional real vector space $\mathfrak{a}=\mathfrak{a}_{J} \oplus \mathfrak{a}_{r}$, a contradiction.

Thus, $w_{i} \neq 0$ and so we must have $h^{i}\left(X_{1}, X_{1}\right)=0$. But then $h^{i}\left(X_{2}, X_{2}\right)=$ $\frac{b_{2}-w_{i}}{a} h^{i}\left(X_{1}, X_{2}\right)$ and again (4.10) is satisfied.

Finally, we need to consider the case $\operatorname{Re}\left(z_{i}\right)=-a / 2$, i.e. $2 \operatorname{Re}\left(z_{i}\right)+a=0$. Then (4.9) is equivalent to $h^{i}\left(X_{1}, X_{2}\right)=\frac{\overline{w_{i}}}{\bar{z}_{i}} h^{i}\left(X_{1}, X_{1}\right)$ and the first equation in (4.7) yields

$$
h^{i}\left(X_{2}, X_{1}\right)=\frac{w_{i} \bar{z}_{i}-a \bar{w}_{i}}{\left|z_{i}\right|^{2}-a \bar{z}_{i}} h^{i}\left(X_{1}, X_{1}\right)
$$

whereas the second equation in (4.7) gives us

$$
h^{i}\left(X_{2}, X_{2}\right)=\frac{\left|w_{i}\right|^{2}-b_{1} \bar{z}_{i}-b_{2} \bar{w}_{i}}{\left|z_{i}\right|^{2}-a \bar{z}_{i}} h^{i}\left(X_{1}, X_{1}\right)
$$

Finally, we need to consider (3.2) on $\Lambda^{2} \mathfrak{a}_{r} \wedge \Lambda^{2} U_{r}$. Here, we get

$$
\begin{aligned}
& 6 v_{1}(\omega)\left(X_{1}, X_{2}, J X_{1}, J X_{2}\right) \\
& \quad=2 a\left(a-b_{1}\right)+\left\|h\left(X_{1}, X_{2}\right)-h\left(X_{2}, X_{1}\right)\right\|^{2}+\left\|h\left(X_{1}, X_{2}\right)\right\|^{2} \\
& \quad+\left\|h\left(X_{2}, X_{1}\right)\right\|^{2}-2 g\left(h\left(X_{1}, X_{1}\right), h\left(X_{1}, X_{2}\right)\right),
\end{aligned}
$$

whereas

$$
\begin{aligned}
12 & v_{1}(\omega)\left(X_{1}, X_{2}, J X_{1}, J X_{2}\right) \\
= & -g\left(\omega\left(J \omega\left(X_{1}, J X_{1}\right), X_{2}\right), X_{2}\right)+g\left(\omega\left(J \omega\left(X_{1}, J X_{2}\right), X_{1}\right), X_{2}\right) \\
& +g\left(\omega\left(J \omega\left(X_{2}, J X_{1}\right), X_{2}\right), X_{1}\right)-g\left(\omega\left(J \omega\left(X_{2}, J X_{2}\right), X_{1}\right), X_{1}\right) \\
= & g\left(f\left(f\left(X_{1}, X_{1}\right), X_{2}\right), X_{2}\right)-g\left(f\left(f\left(X_{2}, X_{1}\right), X_{1}\right), X_{2}\right) \\
& \quad-g\left(f\left(f\left(X_{1}, X_{2}\right), X_{2}\right), X_{1}\right)+g\left(f\left(f\left(X_{2}, X_{2}\right), X_{1}\right), X_{1}\right) \\
= & 0,
\end{aligned}
$$

since $f(f(\cdot, \cdot), \cdot)$ is totally symmetric in its arguments. Hence, (3.2) is true on $\Lambda^{2} \mathfrak{a}_{r} \wedge \Lambda^{2} U_{r}$ if and only if

$$
\begin{gathered}
2 a\left(a-b_{1}\right)+\left\|h\left(X_{1}, X_{2}\right)-h\left(X_{2}, X_{1}\right)\right\|^{2}+\left\|h\left(X_{1}, X_{2}\right)\right\|^{2} \\
+\left\|h\left(X_{2}, X_{1}\right)\right\|^{2}-2 g\left(h\left(X_{1}, X_{1}\right), h\left(X_{1}, X_{2}\right)\right)=0 .
\end{gathered}
$$


Unfortunately, (4.11) seems not to be solvable in a nice and short form in general.

Summarizing, we have obtained:

Theorem 4.10 Let $(\mathfrak{g}, g, J)$ be a two-step solvable $2 n$-dimensional SKT Lie algebra with $\mathfrak{g}^{\prime}$ being non-complex and of codimension two. Then $(\mathfrak{g}, g, J)$ is isomorphic to $\mathfrak{g}_{f, h, \alpha}$ with $\left(X_{1}, X_{2}\right)$ being an orthonormal basis of $\mathfrak{a}_{r}, f\left(X_{1}, X_{j}\right)=a X_{j}$ for $j=1,2$ and $f\left(X_{2}, X_{2}\right)=$ $b_{1} X_{1}+b_{2} X_{2}$ for certain $a>0, b_{1} \in \mathbb{R}, b_{2} \geq 0$ and $\alpha\left(X_{1}\right)=\sum_{i=1}^{n-2} z_{i} Y_{i}, \alpha\left(X_{2}\right)=$ $\sum_{i=1}^{n-2} w_{i} Y_{i}$ for $\left(z_{1}, \ldots, z_{n-2}\right),\left(w_{1}, \ldots, w_{n-2}\right) \in \mathbb{C}^{n-2}$ such that $(4.11)$ is valid and for all $i=1, \ldots, n-2$ we have one of the following three cases:

(i) $\operatorname{Re}\left(z_{i}\right)=0, \operatorname{Re}\left(w_{i}\right)=0, z_{i} \neq 0$ and

$$
\begin{aligned}
& h^{i}\left(X_{2}, X_{1}\right)=h^{i}\left(X_{1}, X_{2}\right)=\frac{w_{i}}{z_{i}} h^{i}\left(X_{1}, X_{1}\right), \\
& h^{i}\left(X_{2}, X_{2}\right)=\frac{w_{i}^{2}-b_{2} w_{i}-b_{1} z_{i}}{z_{i}\left(z_{i}-a\right)} h^{i}\left(X_{1}, X_{1}\right),
\end{aligned}
$$

(ii) $z_{i}=0, \operatorname{Re}\left(w_{i}\right)=0, w_{i} \neq 0$ and

$$
h^{i}\left(X_{1}, X_{1}\right)=0, \quad h^{i}\left(X_{2}, X_{1}\right)=h^{i}\left(X_{1}, X_{2}\right), \quad h^{i}\left(X_{2}, X_{2}\right)=\frac{b_{2}-w_{i}}{a} h^{i}\left(X_{1}, X_{2}\right),
$$

(iii) $\operatorname{Re}\left(z_{i}\right)=-a / 2, \operatorname{Re}\left(w_{i}\right)$ is a solution of $2 x^{2}+b_{2} x-\frac{b_{1}}{2} a=0$ and

$$
\begin{aligned}
& h^{i}\left(X_{2}, X_{1}\right)=\frac{w_{i} \bar{z}_{i}-a \bar{w}_{i}}{\left|z_{i}\right|^{2}-a \bar{z}_{i}} h^{i}\left(X_{1}, X_{1}\right), \\
& h^{i}\left(X_{2}, X_{2}\right)=\frac{\left|w_{i}\right|^{2}-b_{1} \bar{z}_{i}-b_{2} \bar{w}_{i}}{\left|z_{i}\right|^{2}-a \bar{z}_{i}} h^{i}\left(X_{1}, X_{1}\right),
\end{aligned}
$$

where this case may only occur if $b_{1} \geq-b_{2}^{2} /(4 a)$.

Conversely, all such $\mathfrak{g}_{f, h, \alpha}$ are two-step solvable SKT Lie algebras with $\mathfrak{g}^{\prime}$ being non-complex and of codimension two.

Remark 4.11 In Theorem 4.10, we may always choose $h \equiv 0$. Then (4.11) is valid if and only if $b_{1}=a$ and so $a=b_{1} \geq-b_{2}^{2} /(4 a)$, i.e. $4 a^{2} \geq-b_{2}^{2}$, is true. Thus, also case (iii) may occur. Moreover, setting $b:=b_{2}$, the solutions of $2 x^{2}+b x-\left(a^{2} / 2\right)=2 x^{2}+b_{2} x-\left(b_{1} / 2\right) a=0$ are given by $x=\left(-b \pm \sqrt{b^{2}+4 a^{2}}\right) / 4$.

In this case, there are $0 \leq r_{1} \leq r_{2} \leq r_{3} \leq n-2, c_{r_{1}}, \ldots, c_{n-1}, d_{1}, \ldots, d_{n-1}$ such that the only non-zero Lie brackets (up to anti-symmetry and complex-linear extension to $\mathfrak{a}_{J}$ ) are given by

$$
\begin{aligned}
& {\left[J X_{2}, Y_{j}\right]=i d_{j} Y_{j}, j=1, \ldots, r_{1},} \\
& {\left[J X_{1}, Y_{k}\right]=i c_{k} Y_{k}, \quad\left[J X_{2}, Y_{k}\right]=i d_{k} Y_{k}, \quad k=r_{1}+1, \ldots, r_{2},} \\
& {\left[J X_{1}, Y_{\ell}\right]=\left(-\frac{a}{2}+i c_{\ell}\right) Y_{\ell}, \quad\left[J X_{2}, Y_{\ell}\right]=\left(\frac{-b+\sqrt{b^{2}+4 a^{2}}}{4}+i d_{\ell}\right) Y_{\ell},} \\
& \quad \ell=r_{2}+1, \ldots, r_{3}, \\
& {\left[J X_{1}, Y_{m}\right]=\left(-\frac{a}{2}+i c_{m}\right) Y_{m}, \quad\left[J X_{2}, Y_{m}\right]=\left(\frac{-b-\sqrt{b^{2}+4 a^{2}}}{4}+i d_{m}\right) Y_{m},} \\
& \\
& m=r_{3}+1, \ldots, n-2,
\end{aligned}
$$

with $a>0, b \geq 0$. The Lie algebra is unimodular precisely when $r_{2}=n-4$ and $r_{3}=n-3$. 


\section{Commutator ideal totally real}

Here, we look at two-step SKT shear data $(\mathfrak{a}, \omega)$ with totally real $\mathfrak{a}$, i.e. $\mathfrak{a}=\mathfrak{a}_{r}$ and $\mathfrak{a}_{J}=0$, and with $\operatorname{im}(\omega)=\mathfrak{a}$. We note that then the shear Lie algebra $\mathfrak{g}$ has a totally real commutator ideal $\mathfrak{g}^{\prime}$, i.e. we have $\mathfrak{g}^{\prime} \cap J \mathfrak{g}^{\prime}=\{0\}$.

Notation We simplify the notation and, for $Y \in U_{J}$, define the endomorphisms $\alpha_{Y}, \beta_{Y} \in$ $\operatorname{End}(\mathfrak{a})$ by

$$
\alpha_{Y}(X):=\omega(Y, X)=\left(\omega_{0}\right)_{J r}^{r}(Y, X), \quad \beta_{Y}(X):=\omega(Y, J X)=\left(\omega_{1}\right)_{J r}^{r}(Y, J X)
$$

for $X \in \mathfrak{a}$. Moreover, we set

$$
v:=\left(\omega_{1}\right)_{J J}=\left(\omega_{1}\right)_{J J}^{r} \in \Lambda^{2} U_{J}^{*} \otimes \mathfrak{a} .
$$

Moreover, we also use some notation introduced previously without further notification.

We observe the following:

Proposition 5.1 Let $(\mathfrak{a}, \omega)$ be pre-shear data with $\mathfrak{a}$ being totally real. Then $(\mathfrak{a}, \omega)$ is two-step SKT shear data if and only if $f \in S^{2} \mathfrak{a}^{*} \otimes \mathfrak{a},\left(\omega_{1}\right)_{r r}^{r}=0, \beta_{Y}=\alpha_{J Y}$ for all $Y \in U_{J}, v$ is of type $(1,1)$ and

$$
\mathcal{A}(\omega(\omega(\cdot, \cdot), \cdot))=0, \quad \mathcal{A}(g(\omega(\cdot, \cdot), \omega(\cdot, \cdot)))=0 .
$$

Proof All the conditions but (5.1) follow from Lemma 3.3.

Next, note that $J^{*} \omega$ and $\omega$ both have values in $\mathfrak{a}=\mathfrak{a}_{r}$ while $J \circ J . \omega$ has values in $J \mathfrak{a}=J \mathfrak{a}_{r}=U_{r}$. Hence, (3.1) is equivalent $J^{*} \omega=\omega$, since this equation says that $\omega$ is of type $(1,1)$ and so $J . \omega=0$. Thus, $\omega(J \omega(\cdot, \cdot), J \cdot)=\omega(\omega(\cdot, \cdot), \cdot)$, i.e. $v_{2}(\omega)=$ $\mathcal{A}(\omega(\omega(\cdot, \cdot), \cdot))=0$, and $v_{1}(\omega)=\mathcal{A}(g(\omega(\cdot, \cdot), \omega(\cdot, \cdot)))$. Hence, (2.1) and (3.2) are satisfied if and only if

$$
\mathcal{A}(\omega(\omega(\cdot, \cdot), \cdot))=0, \quad \mathcal{A}(g(\omega(\cdot, \cdot), \omega(\cdot, \cdot)))=0 .
$$

Remark 5.2 Note that $J^{*} \omega=\omega$ implies that the induced almost complex structure on the shear Lie algebra is Abelian.

We first look at the exact form of $f$. For this, observe that $f \in S^{2} \mathfrak{a}^{*} \otimes \mathfrak{a}$ and $f(f(\cdot, \cdot), \cdot) \in S^{3} \mathfrak{a}^{*} \otimes V$. Moreover, note that the second equation in (5.1) on $U_{r}^{*} \otimes$ $U_{r}^{*} \otimes \mathfrak{a}^{*} \otimes \mathfrak{a}^{*}$ is equivalent to $g(f(\cdot, \cdot), f(\cdot, \cdot)) \in\left(\mathfrak{a}^{*}\right)^{\otimes 4}$ being totally symmetric.

Such an $f$ can always be brought into a certain "normal form":

Lemma 5.3 Let $(V, g)$ be an m-dimensional Euclidean vector space and $f \in S^{2} V^{*} \otimes V$ such that both $f(f(\cdot, \cdot), \cdot) \in S^{2} V^{*} \otimes V^{*} \otimes V$ and $g(f(\cdot, \cdot), f(\cdot, \cdot)) \in S^{2} V^{*} \otimes$ $S^{2} V^{*}$ are totally symmetric in their $V^{*}$-factors. Set $V_{1}:=\operatorname{im}\left(\left.f\right|_{S^{2}} \operatorname{im}(f), V_{2}:=V_{1}^{\perp} \cap \operatorname{im}(f)\right.$ and $r:=\operatorname{dim}\left(V_{1}\right)$. Then there exist an orthonormal basis $\left(X_{1}, \ldots, X_{r}\right)$ of $V_{1}$, non-zero $\lambda_{1}, \ldots, \lambda_{r} \in \mathbb{R} \backslash\{0\}$ and a complementary subspace $V_{3}$ of $V_{1} \oplus V_{2}$ in $V$ which is orthogonal to $V_{2}$ satisfying the following conditions:

(i) $f\left(X_{a}, X_{b}\right)=\delta_{a b} \lambda_{a} X_{a}$ for all $a, b \in\{1, \ldots, r\}$,

(ii) $f\left(V_{2} \oplus V_{3}, V_{1} \oplus V_{2}\right)=0$ 
(iii) $f\left(V_{3}, V_{3}\right)=V_{2}$.

Proof Let $W=\operatorname{im}(f)$ be the image of $f$. Then our assumptions imply

$$
\left.g(f(\cdot, \cdot), \cdot)\right|_{W \otimes 3} \in S^{3} W^{*},
$$

i.e. is totally symmetric, since $g(f(\cdot, f(\cdot, \cdot)), f(\cdot, \cdot)) \in S^{5}\left(V^{*}\right)$. Hence, $f_{X}:=$ $\left.f(X, \cdot)\right|_{W} \in \operatorname{End}(W)$ is self-adjoint with respect to $g$ for all $X \in \tilde{W}$. Now the symmetry assumption implies that all the endomorphisms $f_{X}$ commute. It follows that $W$ has a common orthonormal basis $X_{1}, \ldots, X_{m}$ of eigenvectors for these endomorphisms. Label these vectors so that $f\left(X_{i}, X_{i}\right) \neq 0$ for $i=1, \ldots, r$. As $f_{X_{a}}\left(X_{b}\right)=f\left(X_{a}, X_{b}\right)=$ $f_{X_{b}}\left(X_{a}\right) \in \operatorname{span}\left(X_{a}\right) \cap \operatorname{span}\left(X_{b}\right)$ for all $a, b \in\{1, \ldots, m\}$, we find that $f\left(X_{a}, X_{b}\right)$ is only non-zero for $a=b \leq r$. Thus we get $W=V_{1} \oplus V_{2}$ orthogonally, with $V_{1}=\operatorname{im}\left(\left.f\right|_{S^{2} W}\right)=\operatorname{span}\left(X_{1}, \ldots, X_{r}\right)$ and $V_{2}=\operatorname{span}\left(X_{r+1}, \ldots, X_{m}\right)$, giving (i) and $f\left(V_{2}, V_{1} \oplus V_{2}\right)=0$ in (ii).

Note that for $Y \in V_{2}$ and $Z \in V$, we have

$$
g(f(Y, Z), f(Y, Z))=g(f(Y, Y), f(Z, Z))=0,
$$

which implies that $f(Y, Z)=f(Z, Y)=0$ for any $Z \in V$. Next, let $H: W^{\perp} \rightarrow$ $V, H(Y):=Y-\sum_{i=1}^{r} \frac{1}{\lambda_{i}} f\left(Y, X_{i}\right)$ and note that $H$ is an injective linear map with $f\left(H(Y), X_{i}\right)=0$ for all $i=1 \ldots, r$. Hence, $V_{3}:=H\left(W^{\perp}\right)$ is a complement of $W$ in $V$ with $f\left(V_{3}, W\right)=0$, and so (ii) holds. Note that $V_{3}$ is not necessarily orthogonal to $V_{1}$ but it is orthogonal to $V_{2}$. However, we get

$$
g\left(X_{i}, f(Y, Z)\right)=\frac{1}{\lambda_{i}} g\left(f\left(X_{i}, X_{i}\right), f(Y, Z)\right)=\frac{1}{\lambda_{i}} g\left(f\left(X_{i}, Y\right), f\left(X_{i}, Z\right)\right)=0
$$

for all $i=1, \ldots, r$ and all $Y, Z \in V_{3}$, which implies that $f(Y, Z) \in V_{1}^{\perp} \cap(\operatorname{im}(f))=V_{2}$, as claimed in (iii).

In our case, i.e. the case of two-step shear data $(\mathfrak{a}, \omega)$ with totally real $\mathfrak{a}$, we get $\mathfrak{a}_{2}=\{0\}$ in Lemma 5.3 and even more the following is true:

Proposition 5.4 Let $(\mathfrak{a}, \omega)$ be two-step SKT shear data with totally real $\mathfrak{a}$. Then, with the notation from the beginning of this subsection, we have $\mathfrak{a}=\mathfrak{a}_{1} \oplus \mathfrak{a}_{3}$, an orthonormal basis $X_{1}, \ldots, X_{r}$ of $\mathfrak{a}_{1}, r:=\operatorname{dim}\left(\mathfrak{a}_{1}\right), \lambda_{1}, \ldots, \lambda_{r} \in \mathbb{R} \backslash\{0\}$ and one-forms $\mu_{1}, \ldots, \mu_{r}$ such that

$$
f\left(X_{i}, X_{j}\right)=\delta_{i j} \lambda_{i} X_{i}, \quad f\left(\mathfrak{a}_{3}, \mathfrak{a}\right)=0, \quad \alpha_{Y}\left(X_{i}\right)=\mu_{i}(Y) X_{i}, \quad \alpha_{Y}\left(\mathfrak{a}_{3}\right)=0
$$

for all $i, j \in\{1, \ldots, r\}$.

Proof Let $\mathfrak{a}_{1}, \mathfrak{a}_{2}, \mathfrak{a}_{3}, r, s,\left(X_{1}, \ldots, X_{r}\right), \lambda_{1}, \ldots, \lambda_{r} \in \mathbb{R} \backslash\{0\}$ be as in Lemma 5.3 and let us first look at $\alpha \in U_{J}^{*} \otimes \operatorname{End}(\mathfrak{a})$.

Then the first condition in (5.1) gives us that $\alpha_{Y} \in \operatorname{End}(\mathfrak{a})$ commutes with $f_{X}:=f(X, \cdot)$ for any $X \in \mathfrak{a}$. In particular, $\alpha_{Y}$ preserves the eigenspaces of all $f_{X_{i}}$ and so $\operatorname{span}\left(X_{i}\right)$ for all $i=1, \ldots, r$ as well as $\mathfrak{a}_{2} \oplus \mathfrak{a}_{3}$, which is the common zero eigenspace of all $f_{X_{i}}, i=1, \ldots, r$. In particular, there are $\mu_{1}, \ldots, \mu_{r} \in U_{J}^{*}$ with $\alpha_{Y}\left(X_{i}\right)=\mu_{i}(Y) X_{i}$ for all $i=1, \ldots, r$.

Next, look at the second equation in (5.1) and insert into that equation $(X, J X, Y, J Y)$ with $X \in \mathfrak{a}_{2}$ As $f(X, X)=0$, one gets

$$
\begin{aligned}
0 & =\mathcal{A}(g(\omega(\cdot, \cdot), \omega(\cdot, \cdot)))(X, J X, Y, J Y) \\
& =-g(\omega(X, Y), \omega(J X, J Y))+g(\omega(X, J Y), \omega(J X, Y)) \\
& =-g\left(\alpha_{Y}(X), \beta_{J Y}(X)\right)+g\left(\alpha_{J Y}(X), \beta_{Y}(J X)\right)=-\left\|\alpha_{Y}(X)\right\|^{2}-\left\|\alpha_{J Y}(X)\right\|^{2},
\end{aligned}
$$


i.e. $\alpha_{Y}(X)=0$ and so $\alpha_{Y}\left(\mathfrak{a}_{2}\right)=0$, where here and in the following we leave out irrelevant normalization factors coming from anti-symmetrization map $\mathcal{A}$.

Now take $X, W \in \mathfrak{a}_{3}$. Then $0=\left[f_{X}, \alpha_{Y}\right](W)=f_{X}\left(\alpha_{Y}(W)\right)$ since $f_{X}(W) \in \mathfrak{a}_{2} \subseteq$ $\operatorname{ker}\left(\alpha_{Y}\right)$. Hence, if we denote by $\mathfrak{a}_{3}^{\prime}$ the common kernel of all $\left.f_{X}\right|_{\mathfrak{a}_{3}}, X \in \mathfrak{a}_{3}$, then $\alpha_{Y}\left(\mathfrak{a}_{3}\right) \subseteq$ $\mathfrak{a}_{2} \oplus \mathfrak{a}_{3}^{\prime}$. We are going to show that $\mathfrak{a}_{3}^{\prime}=\mathfrak{a}_{3}$, which implies $f_{X}=0$ for all $X \in \mathfrak{a}_{3}$ and so $\mathfrak{a}_{2}=0$ by condition (iii) in Lemma 5.3.

For this, let $X \in \mathfrak{a}_{3}^{\prime}$ and $Y \in U_{J}$. Again, we have $f(X, X)$ and so

$$
\begin{aligned}
0 & =\mathcal{A}(g(\omega(\cdot, \cdot), \omega(\cdot, \cdot)))(X, J X, Y, J Y) \\
& =-g\left(\alpha_{Y}(X), \beta_{J Y}(X)\right)+g\left(\alpha_{J Y}(X), \beta_{Y}(J X)\right)=-\left\|\alpha_{Y}(X)\right\|^{2}-\left\|\alpha_{J Y}(X)\right\|^{2} .
\end{aligned}
$$

i.e. $\alpha_{Y}\left(\mathfrak{a}_{3}^{\prime}\right)=\{0\}$, and so $\alpha_{Y}\left(\mathfrak{a}_{2} \oplus \mathfrak{a}_{3}^{\prime}\right)=\{0\}$.

Next let $X \in \mathfrak{a}_{3}, Y, Z \in U_{J}$ be given. Then (5.1) gives us

$$
\begin{aligned}
0 & =\mathcal{A}(\omega(\omega(\cdot, \cdot), \cdot))(J X, Y, Z)=f(X, v(Y, Z))-\alpha_{Y}\left(\alpha_{J Z}(X)\right)+\alpha_{Z}\left(\alpha_{J Y}(X)\right) \\
& =f(X, v(Y, Z))
\end{aligned}
$$

since $\alpha_{J Z}(X), \alpha_{J Y}(X) \in \mathfrak{a}_{2} \oplus \mathfrak{a}_{3}^{\prime}$.

But this implies $v(Y, Z) \in \mathfrak{a}_{1} \oplus \mathfrak{a}_{2} \oplus \mathfrak{a}_{3}^{\prime}$ for any $Y, Z \in U_{J}$. As the images of $f, \alpha_{Y}$ and $\beta_{Z}$ are all $\mathfrak{a}_{1} \oplus \mathfrak{a}_{2} \oplus \mathfrak{a}_{3}^{\prime}$ for any $Y, Z \in U_{J}$, we have $\operatorname{im}(\omega) \subseteq \mathfrak{a}_{1} \oplus \mathfrak{a}_{2} \oplus \mathfrak{a}_{3}^{\prime}$. However, by assumption, $\operatorname{im}(\omega)=\mathfrak{a}=\mathfrak{a}_{1} \oplus \mathfrak{a}_{2} \oplus \mathfrak{a}_{3}$, and so $\mathfrak{a}_{3}^{\prime}=\mathfrak{a}_{3}$. As said above, this implies $\mathfrak{a}_{2}=\{0\}$. Note that now also $0=\alpha_{Y}\left(\mathfrak{a}_{3}^{\prime}\right)=\alpha_{Y}\left(\mathfrak{a}_{3}\right)$ for any $Y \in U_{J}$, which finishes the proof.

We write now $v=\sum_{i=1}^{r} v_{i} X_{i}+\tilde{v}$ with $v_{1}, \ldots v_{r} \in \Lambda^{2} U_{J}^{*}$ and $\tilde{v} \in \Lambda^{2} U_{J}^{*} \otimes \mathfrak{a}_{3}$. Then, applying the first part of (5.1) to $\left(J X_{i}, Y, Z\right)$ with $i \in\{1, \ldots, r\}$ and $Y, Z \in U_{J}$, one obtains

$$
\begin{aligned}
0 & =\mathcal{A}(\omega(\omega(\cdot, \cdot), \cdot))\left(J X_{i}, Y, Z\right)=f\left(X_{i}, v(Y, Z)\right)-\left(\mu_{i} \wedge \mu_{i} \circ J\right)(Y, Z) X_{i} \\
& =\left(\lambda_{i} v_{i}-\left(\mu_{i} \wedge \mu_{i} \circ J\right)\right)(Y, Z) X_{i},
\end{aligned}
$$

so $v_{i}=\left(\mu_{i} \wedge \mu_{i} \circ J\right) / \lambda_{i}$.

Next, we show that the splitting $\mathfrak{a}=\mathfrak{a}_{1} \oplus \mathfrak{a}_{3}$ is $g$-orthogonal. For this, we insert $\left(J X_{i}, X_{i}, Y, Z\right)$ with $i \in\{1, \ldots, r\}$ and $Y, Z \in U_{J}$ into the second equation in (5.1) and get

$$
\begin{aligned}
0 & =\mathcal{A}(g(\omega(\cdot, \cdot), \omega(\cdot, \cdot)))\left(J X_{i}, X_{i}, Y, Z\right) \\
& =g\left(f\left(X_{i}, X_{i}\right), v(Y, Z)\right)+g\left(\alpha_{J Y}\left(X_{i}\right), \alpha_{Z}\left(X_{i}\right)\right)-g\left(\alpha_{J Z}\left(X_{i}\right), \alpha_{Y}\left(X_{i}\right)\right) \\
& =\left(\mu_{i} \wedge \mu_{i} \circ J\right)(Y, Z)+\lambda_{i} g\left(X_{i}, \tilde{v}(Y, Z)\right)-\left(\mu_{i} \wedge \mu_{i} \circ J\right)(Y, Z) \\
& =\lambda_{i} g\left(X_{i}, \tilde{v}(Y, Z)\right),
\end{aligned}
$$

thus $\tilde{v}(Y, Z) \perp_{g} \mathfrak{a}_{1}$ for all $Y, Z \in U_{J}$. As by assumption $\operatorname{im}(\omega)=\mathfrak{a}$ and so $\mathfrak{a}_{3}$ has to be spanned by $\operatorname{im}(\tilde{v})$, we obtain $\mathfrak{a}_{1} \perp_{g} \mathfrak{a}_{3}$. Now one checks that (5.1) is valid with the exception of the second equation on $\Lambda^{4} U_{J}^{*}$, which is equivalent to $\sum_{i=r+1}^{m} \tilde{v}_{i} \wedge \tilde{v}_{i}=0$ if we write $\tilde{v}=\sum_{i=r+1}^{m} \tilde{v}_{i} X_{i}$ for an orthonormal basis $X_{r+1}, \ldots, X_{m}$ of $\mathfrak{a}_{3}$ with $\tilde{v}_{r+1}, \ldots, \tilde{v}_{m} \in \Lambda^{2} U_{J}^{*}$. Summarizing, we have obtained:

Theorem 5.5 Let $(\mathfrak{g}, g, J)$ be a $2 n$-dimensional almost Hermitian two-step solvable Lie algebra with totally real m-dimensional commutator ideal $\mathfrak{g}^{\prime}$.

Then $(\mathfrak{g}, g, J)$ is SKT if and only if there exists an orthonormal basis $\left(X_{1}, \ldots, X_{m}\right)$ of $\mathfrak{g}^{\prime}, r \in\{0, \ldots, m\}, \lambda_{1}, \ldots, \lambda_{r} \in \mathbb{R} \backslash\{0\}, \mu_{1}, \ldots, \mu_{r} \in U_{J}^{*}$ with $U_{J}:=\left(\mathfrak{g}^{\prime} \oplus J \mathfrak{g}^{\prime}\right)^{\perp}$ and 
$\tilde{v}_{r+1}, \ldots, \tilde{v}_{m} \in \Lambda^{2} U_{J}^{*}$ such that the only non-zero Lie brackets (up to anti-symmetry) are given by

$$
\begin{aligned}
{\left[J X_{i}, X_{i}\right] } & =\lambda_{i} X_{i},\left[Y, X_{i}\right]=\mu_{i}(Y) X_{i},\left[Y, J X_{i}\right]=-\mu_{i}(J Y) X_{i}, \\
{[Y, Z] } & =\sum_{i=1}^{r} \frac{1}{\lambda_{i}}\left(\mu_{i} \wedge \mu_{i} \circ J\right)(Y, Z) X_{i}+\sum_{j=r+1}^{m} \tilde{v}_{j}(Y, Z) X_{j},
\end{aligned}
$$

for $i=1, \ldots, r, Y, Z \in U_{J}$, and such that for $\tilde{v}:=\sum_{i=r+1}^{m} \tilde{v}_{i} X_{i}$ we have $\sum_{i=r+1}^{m} \tilde{v}_{i} \wedge \tilde{v}_{i}=$ 0 and $\operatorname{im}(\tilde{v})=\operatorname{span}\left(X_{r+1}, \ldots, X_{m}\right)$.

If this is the case, we have $\mathfrak{g} \cong r \mathfrak{a f f} \mathfrak{R}_{\mathbb{R}} \oplus \mathfrak{h}$ for some $2(n-r)$-dimensional nilpotent Lie algebra $\mathfrak{h}$.

Remark 5.6 Observe that by Theorem 5.5 and in stark contrast to the Lie algebras considered in Sect. 4, a unimodular two-step solvable Lie algebra with totally real commutator ideal is automatically nilpotent. Moreover, we then have $\left[\mathfrak{g}^{\prime}, \mathfrak{g}\right]=0$, i.e. $\mathfrak{g}$ is two-step nilpotent. This confirms the conjecture by Enrietti, Fino and Vezzoni $[7,13]$ that any nilpotent SKT Lie algebra is two-step nilpotent in the particular case of a nilpotent, two-step solvable SKT Lie algebra with totally real commutator ideal.

If $m=r$ in Theorem 5.5, then $\tilde{v}$ does not occur anymore and we have the following result.

Corollary 5.7 Let $(\mathfrak{g}, g, J)$ be a 2 n-dimensional almost Hermitian two-step solvable Lie algebra with totally real m-dimensional commutator ideal $\mathfrak{g}^{\prime}$ such that $\left[\mathfrak{g}^{\prime}, J \mathfrak{g}^{\prime}\right]=\mathfrak{g}^{\prime}$.

Then $(\mathfrak{g}, g, J)$ is SKT if and only if there exists an orthonormal basis $\left(X_{1}, \ldots, X_{m}\right)$ of $\mathfrak{g}^{\prime}$, $\lambda_{1}, \ldots, \lambda_{m} \in \mathbb{R} \backslash\{0\}, \mu_{1}, \ldots, \mu_{m} \in U_{J}^{*}$ with $U_{J}:=\left(\mathfrak{g}^{\prime} \oplus J \mathfrak{g}^{\prime}\right)^{\perp}$, such that the only non-zero Lie brackets (up to anti-symmetry) are given by

$$
\begin{aligned}
{\left[J X_{i}, X_{i}\right] } & =\lambda_{i} X_{i}, \quad\left[Y, X_{i}\right]=\mu_{i}(Y) X_{i}, \quad\left[Y, J X_{i}\right]=-\mu_{i}(J Y) X_{i}, \\
{[Y, Z] } & =\sum_{i=1}^{m} \frac{1}{\lambda_{i}}\left(\mu_{i} \wedge \mu_{i} \circ J\right)(Y, Z) X_{i},
\end{aligned}
$$

for $i=1, \ldots, m, Y, Z \in U_{J}$, If this is the case, we have $\mathfrak{g} \cong m \mathfrak{a f f} \mathfrak{R}_{\mathbb{R}} \oplus \mathbb{R}^{2 n-2 m}$.

For $n=m$ in Corollary 5.7 we get:

Corollary 5.8 A 2n-dimensional almost Hermitian two-step solvable Lie algebra $(\mathfrak{g}, g, J)$ with totally real commutator ideal $\mathfrak{g}^{\prime}$ of dimension $n$ is SKT if and only if there exists an orthonormal basis $\left(X_{1}, \ldots, X_{n}\right)$ of $\mathfrak{g}^{\prime}$ and $\lambda_{1}, \ldots, \lambda_{n} \in \mathbb{R} \backslash\{0\}$ such that the only non-zero Lie brackets (up to anti-symmetry) are given by $\left[J X_{i}, X_{i}\right]=\lambda_{i} X_{i}$ for $i=1, \ldots, n$

Moreover, we then have $\mathfrak{g} \cong n \mathfrak{a f f} \mathfrak{R}_{\mathbb{R}}$.

For $r<m$, we still need to solve $\sum_{i=r+1}^{m} \tilde{v}_{i} \wedge \tilde{v}_{i}=0$ and $\operatorname{im}(\tilde{v})=\operatorname{span}\left(X_{r+1}, \ldots\right.$, $\left.X_{m}\right)$ in Theorem 5.5. This is not too difficult for $r \in\{m-2, m-1\}$, and so we will do that in the following. For smaller $r$, solving these equations seems to be more involved, cf. Remark 5.11.

Let us start with $r=m-1$. Then $\tilde{v}_{m} \wedge \tilde{v}_{m}=0, \operatorname{im}(\tilde{v})=\operatorname{span}\left(X_{m}\right)$ and $\tilde{v}_{m}$ being a (1, 1)-form implies $\tilde{v}_{m}=\alpha \wedge J^{*} \alpha$ for some $\alpha \in U_{J}^{*} \backslash\{0\}$.

Next, consider the case $r=m-2$. Then we need to solve the equation $\tilde{v}_{m-1}^{2}+\tilde{v}_{m}^{2}=0$, which is done by the next lemma. 
Lemma 5.9 Let $V$ be a $2 n$-dimensional vector space with complex structure $J$ and let real $v_{1}, v_{2} \in \Lambda^{1,1} V^{*}$ be given with $v_{1}^{2}+v_{2}^{2}=0$. Then, up to a rotation in $\operatorname{span}\left(v_{1}, v_{2}\right)$, there are $\alpha, \beta \in V^{*}$ such that $v_{1}=\alpha \wedge J^{*} \alpha$ and $\nu_{2}=\beta \wedge J^{*} \beta$.

Proof First of all, we show that if $v_{1}^{2}+v_{2}^{2}=0$ implies then both $v_{1}$ and $v_{2}$ has rank at most four. For this, assume contrary, without loss of generality, that $v_{1}$ has rank at least six. Then we may find a decomposition $V=V_{1} \oplus V_{2}$ with $\operatorname{dim}\left(V_{1}\right)=4$ such that $\nu_{1}=\omega_{1}+\tau_{1}$ with $\omega_{1} \in \Lambda^{2} V_{1}^{*}$ being of rank four and $0 \neq \tau_{1} \in \Lambda^{2} V_{2}^{*}$. We may decompose $\tilde{\mu}_{2}=\omega_{2}+\rho_{2}+\tau_{2}$ with $\omega_{2} \in \Lambda^{2} V_{1}^{*}, \rho_{2} \in V_{1}^{*} \wedge V_{2}^{*}$ and $\tau_{2} \in \Lambda^{2} V_{2}^{*}$. Looking at the $\Lambda^{4} V_{1}^{*}$-component and the $\Lambda^{3} V_{1}^{*} \wedge V_{2}^{*}$-component of $v_{1}^{2}+v_{2}^{2}=0$, we obtain

$$
\omega_{1}^{2}+\omega_{2}^{2}=0, \quad \omega_{2} \wedge \rho_{2}=0
$$

Due to $\omega_{1}^{2} \neq 0$, we must have $\omega_{2} \neq 0$ and so the second equation implies $\rho_{2}=0$. But then the $\Lambda^{2} V_{1}^{*} \wedge \Lambda^{2} V_{2}^{*}$-component of $v_{1}^{2}+v_{2}^{2}=0$ gives us

$$
\omega_{1} \wedge \tau_{1}+\omega_{2} \wedge \tau_{2}=0
$$

As $\omega_{2}^{2}=-\omega_{1}^{2}$, the two-forms $\omega_{1}, \omega_{2}$ cannot be linearly dependent and so we must have $\tau_{1}=\tau_{2}=0$, a contradiction. Hence, both $v_{1}$ and $v_{2}$ have rank at most four. If they both have rank four, then they must have the same kernel since if $X$ is in the kernel of say $v_{1}$, then $\left.0=X\lrcorner\left(v_{1}^{2}+v_{2}^{2}\right)=2(X\lrcorner v_{2}\right) \wedge v_{2}$, which forces $\left.X\right\lrcorner v_{2}=0$ as well. Moreover, it cannot be that exactly one of the two-form $\omega_{2}, \omega_{1}$ has rank four so the only other option is that both have rank two. So in this case, we have $\nu_{1}=\alpha \wedge J^{*} \alpha$ and $\nu_{2}=\beta \wedge J^{*} \beta$ for $\alpha, \beta \in U_{J}^{*}$.

Let us now come back to the case that both $v_{1}$ and $\nu_{2}$ have rank four, and so have common support. Setting $\Omega:=v_{1}^{2}$, we have $v_{2}^{2}=-\Omega$ and $v_{1} \wedge v_{2}=a \Omega$ for some $a \in \mathbb{R}$. As the matrix $\left(\begin{array}{ll}1 & a \\ a & -1\end{array}\right)$ is symmetric, we may apply an orthogonal transformation to $\operatorname{span}\left(X_{1}, X_{2}\right)$ such that with respect to the new basis we get $a=0$. But then $\left(\frac{1}{\sqrt{2}} v_{1} \pm \frac{1}{\sqrt{2}} v_{2}\right)^{2}=0$ and so by applying another orthogonal transformation to $\operatorname{span}\left(v_{1}, v_{2}\right)$, we may also here assume that both $v_{1}$ and $v_{2}$ have rank two. Hence, we always can rotate the pair $\left(v_{1}, v_{2}\right)$ to get $v_{1}=\alpha \wedge J^{*} \alpha$ and $\nu_{2}=\beta \wedge J^{*} \beta$ for certain $\alpha, \beta \in V^{*}$

Coming back to our case, we thus have $\tilde{v}_{m-1}=\alpha \wedge J^{*} \alpha$ and $\tilde{v}_{m}=\beta \wedge J^{*} \beta$ for certain $\alpha, \beta \in U_{J}^{*}$. Additionally, we know that we must have $\operatorname{im}(v)=\operatorname{span}\left(X_{m-1}, X_{m}\right)$. But so $\tilde{v}_{m-1}, \tilde{v}_{m}$ have to be $\mathbb{R}$-linearly independent, which implies that $\alpha, \beta$ are $\mathbb{C}$-linearly independent. Hence, we have obtained:

Theorem 5.10 Let $(\mathfrak{g}, g, J)$ be a 2 n-dimensional almost Hermitian two-step solvable Lie algebra with totally real m-dimensional commutator ideal $\mathfrak{g}^{\prime}$ such that $\left[\mathfrak{g}^{\prime}, J \mathfrak{g}^{\prime}\right]$ has codimension $\ell \in\{1,2\}$ in $\mathfrak{g}^{\prime}$. Moreover, set $U_{J}:=\left(\mathfrak{g}^{\prime} \oplus J \mathfrak{g}^{\prime}\right)^{\perp}$.

(a) If $\ell=1$, then $(\mathfrak{g}, g, J)$ is SKT if and only if there exists an orthonormal basis $\left(X_{1}, \ldots, X_{m}\right)$ of $\mathfrak{g}^{\prime}, \lambda_{1}, \ldots, \lambda_{m-1} \in \mathbb{R} \backslash\{0\}, \mu_{1}, \ldots, \mu_{m-1}, \alpha \in U_{J}^{*}$ with $\alpha \neq 0$ such that the only non-zero Lie brackets (up to anti-symmetry) are given by

$$
\begin{aligned}
{\left[J X_{i}, X_{i}\right] } & =\lambda_{i} X_{i},\left[Y, X_{i}\right]=\mu_{i}(Y) X_{i},\left[Y, J X_{i}\right]=-\mu_{i}(J Y) X_{i}, \\
{[Y, Z] } & =\sum_{i=1}^{m-1} \frac{1}{\lambda_{i}}\left(\mu_{i} \wedge \mu_{i} \circ J\right)(Y, Z) X_{i}+\left(\alpha \wedge J^{*} \alpha\right)(Y, Z) X_{m},
\end{aligned}
$$

for $i=1, \ldots, m-1, Y, Z \in U_{J}$. In this case, we have $\mathfrak{g} \cong(m-1) \mathfrak{a f f} \mathfrak{R}_{\mathbb{R}} \oplus \mathfrak{h}_{3} \oplus$ $\mathbb{R}^{2 n-2 m-1}$. 
(b) If $\ell=2$, then $(\mathfrak{g}, g, J)$ is $S K T$ if and only if there exists an orthonormal basis $\left(X_{1}, \ldots, X_{m}\right)$ of $\mathfrak{g}^{\prime}, \lambda_{1}, \ldots, \lambda_{m-2} \in \mathbb{R} \backslash\{0\}, \mu_{1}, \ldots, \mu_{m-2}, \alpha_{1}, \alpha_{2} \in U_{J}^{*}$ with $\alpha_{1}$, $\alpha_{2}$ being $\mathbb{C}$-linearly independent such that the only non-zero Lie brackets (up to antisymmetry) are given by

$$
\begin{aligned}
{\left[J X_{i}, X_{i}\right] } & =\lambda_{i} X_{i},\left[Y, X_{i}\right]=\mu_{i}(Y) X_{i},\left[Y, X_{i}\right]=-\mu_{i}(J Y) X_{i}, \\
{[Y, Z] } & =\sum_{i=1}^{m-2} \frac{1}{\lambda_{i}}\left(\mu_{i} \wedge \mu_{i} \circ J\right)(Y, Z) X_{i}+\sum_{i=1}^{2}\left(\alpha_{i} \wedge J^{*} \alpha_{i}\right)(Y, Z) X_{m-2+i},
\end{aligned}
$$

for $i=1, \ldots, m-2, Y, Z \in U_{J}$. In this case, we have $\mathfrak{g} \cong(m-2) \mathfrak{a f f} \mathfrak{R}_{\mathbb{R}} \oplus 2 \mathfrak{h}_{3} \oplus$ $\mathbb{R}^{2 n-2 m-2}$.

Remark 5.11 Note that for higher codimensions $\ell$ it is not clear whether Lemma 5.9 generalizes in the natural way to more than two $(1,1)$-forms $v_{i}$. So one is left with the description from Theorem 5.5 for these cases. Note further that even if all $v_{i}$ are decomposable, still the nilpotent Lie algebra $\mathfrak{h}$ from Theorem 5.5 is, in general, not equal to a sum of the form $r \mathfrak{a f f} \mathbb{R}_{\mathbb{R}} \oplus \ell \mathfrak{h}_{3} \oplus \mathbb{R}^{2(n-r)-3 s}$ as in the case of $\ell \in\{1,2\}$.

For example, if $\ell=3$, take $\mathbb{C}$-linearly independent $\alpha_{1}, \alpha_{2} \in U_{J}$ and set $v_{m-2}:=\alpha_{1} \wedge J^{*} \alpha_{1}$, $v_{m-1}:=\alpha_{2} \wedge J^{*} \alpha_{2}, v_{m}:=\left(\alpha_{1}+\alpha_{2}\right) \wedge J^{*}\left(\alpha_{1}+\alpha_{2}\right)$. Then $\mathfrak{h} \cong r \mathfrak{a f f} \mathfrak{f}_{\mathbb{R}} \oplus(37 D) \oplus \mathbb{R}^{2(n-r)-7}$ with the seven-dimensional nilpotent Lie algebra $37 D$ from [16].

Now all Lie algebras up to dimension two are solvable and the only nilpotent Lie algebras up to this dimension are the Abelian ones. As the commutator ideal $\mathfrak{g}^{\prime}$ of a solvable Lie algebra is nilpotent, one sees that any Lie algebra with one-dimensional or two-dimensional commutator ideal is two-step solvable. Now surely if the dimension of $\mathfrak{g}^{\prime}$ is one, $\mathfrak{g}^{\prime}$ is totally real and the codimension of $\left[\mathfrak{g}^{\prime}, J \mathfrak{g}^{\prime}\right]$ in $\mathfrak{g}^{\prime}$ is at most one. Similarly, if the dimension of $\mathfrak{g}^{\prime}$ is two and $\mathfrak{g}^{\prime}$ is totally real, then the codimension of $\left[\mathfrak{g}^{\prime}, J \mathfrak{g}^{\prime}\right]$ in $\mathfrak{g}^{\prime}$ is at most two. Hence, Corollary 5.7 and Theorem 5.10 imply

Corollary 5.12 Let $(\mathfrak{g}, g, J)$ be a $2 n$-dimensional almost Hermitian Lie algebra.

(a) If $\operatorname{dim}\left(\mathfrak{g}^{\prime}\right)=1$, then $(\mathfrak{g}, g, J)$ is an SKT Lie algebra if and only if there exists $0 \neq X \in \mathfrak{g}^{\prime}$ of norm one such that

(i) there exists $\lambda \in \mathbb{R} \backslash\{0\}$ and $\mu \in U_{J}^{*}$ so that the only non-zero Lie brackets (up to anti-symmetry) are given by

$$
\begin{aligned}
& =\lambda X,[Y, X]=\mu(Y) X,[Y, J X]=-\mu(J Y) X \text { for } Y \in U_{J}, \\
{[Y, Z] } & =\frac{1}{\lambda}(\mu \wedge \mu \circ J)(Y, Z) X \text { for } Y, Z \in U_{J}
\end{aligned}
$$

or

(ii) there exists $\alpha \in U_{J}^{*} \backslash\{0\}$ so that the only non-zero Lie bracket is given by

$$
[Y, Z]=\left(\alpha \wedge J^{*} \alpha\right)(Y, Z) X
$$

for $Y, Z \in U_{J}$.

In the first case, we have $\mathfrak{g} \cong \mathfrak{a f f}_{\mathbb{R}} \oplus \mathbb{R}^{2 n-2}$ whereas in the second case we have $\mathfrak{g} \cong \mathfrak{h}_{3} \oplus \mathbb{R}^{2 n-3}$.

(b) If $\operatorname{dim}\left(\mathfrak{g}^{\prime}\right)=2$ and $\mathfrak{g}^{\prime}$ is totally real, then $(\mathfrak{g}, g, J)$ is an SKT Lie algebra if and only if there exists an orthonormal basis $\left(X_{1}, X_{2}\right)$ of $\mathfrak{g}^{\prime}$ such that 
(i) there exist $\lambda_{1}, \lambda_{2} \in \mathbb{R} \backslash\{0\}$ and $\mu_{1}, \mu_{2} \in U_{J}^{*}$ so that the only non-zero Lie brackets (up to anti-symmetry) are given by

$$
\begin{aligned}
{\left[J X_{i}, X_{i}\right] } & =\lambda X,\left[Y, X_{i}\right]=\mu(Y) X_{i},\left[Y, J X_{i}\right]=-\mu(J Y) X_{i} \text { for } i=1,2, Y \in U_{J}, \\
{[Y, Z] } & =\sum_{i=1}^{2} \frac{1}{\lambda_{i}}\left(\mu_{i} \wedge \mu_{i} \circ J\right)(Y, Z) X_{i} \text { for } Y, Z \in U_{J},
\end{aligned}
$$

(ii) there exist $\lambda \in \mathbb{R} \backslash\{0\}$ and $\mu, \alpha \in U_{J}^{*}, \alpha \neq 0$ so that the only non-zero Lie brackets (up to anti-symmetry) are given by

$$
\begin{aligned}
& {\left[J X_{1}, X_{1}\right]=\lambda X_{1},\left[Y, X_{1}\right]=\mu(Y) X_{1},\left[Y, J X_{1}\right]=-\mu(J Y) X_{1} \text { for } Y \in U_{J},} \\
& {[Y, Z]=\frac{1}{\lambda}(\mu \wedge \mu \circ J)(Y, Z) X_{1}+\left(\alpha \wedge J^{*} \alpha\right)(Y, Z) X_{2} \text { for } Y, Z \in U_{J}} \\
& \text { or }
\end{aligned}
$$

(iii) there exist $\mathbb{C}$-linearly independent $\alpha, \beta \in U_{J}^{*}$ so that the only non-zero Lie bracket is given by

$$
[Y, Z]=\left(\alpha \wedge J^{*} \alpha\right)(Y, Z) X_{1}+\left(\beta \wedge J^{*} \beta\right)(Y, Z) X_{2}
$$

for $Y, Z \in U_{J}$.

In the first case, we have $\mathfrak{g} \cong 2 \mathfrak{a} \mathfrak{a f} \mathfrak{R}_{\mathbb{R}} \oplus \mathbb{R}^{2 n-4}$, in the second case, we have $\mathfrak{g} \cong \mathfrak{a f f} \mathfrak{R}_{\mathbb{R}} \oplus$ $\mathfrak{h}_{3} \oplus \mathbb{R}^{2 n-5}$ and in the third case, we have $\mathfrak{g} \cong 2 \mathfrak{h}_{3} \oplus \mathbb{R}^{2 n-6}$.

Remark 5.13 Note that by [1, Proposition 2.2], a $2 n$-dimensional Lie algebras $\mathfrak{g}$ with onedimensional commutator ideal is either isomorphic to $\mathfrak{h}_{2 k+1} \oplus \mathbb{R}^{2(n-k)-1}$ or $\mathfrak{a f f}_{\mathbb{R}} \oplus \mathbb{R}^{2 n-2}$ for some $k \in \mathbb{R}$ and that all these Lie algebras admit an Abelian complex structure $J$. So we have shown that on $\mathfrak{h}_{2 k+1} \oplus \mathbb{R}^{2(n-k)-1}$ with $k \geq 2$ any of these Abelian complex structures cannot come from an SKT structure on $\mathfrak{g}$.

Corollary 5.14 A six-dimensional two-step solvable Lie algebra $\mathfrak{g}$ admits an SKT structure with totally real $\mathfrak{g}^{\prime}$ if and only if $\mathfrak{g}$ is isomorphic to one of the following Lie algebras:

$$
\mathbb{R}^{6}, \mathfrak{a f f} \mathfrak{R}_{\mathbb{R}} \oplus \mathbb{R}^{4}, \mathfrak{h}_{3} \oplus \mathbb{R}^{3}, 2 \mathfrak{a f f} \mathfrak{f}_{\mathbb{R}} \oplus \mathbb{R}, \mathfrak{a f f} \mathfrak{R}_{\mathbb{R}} \oplus \mathfrak{h}_{3} \oplus \mathbb{R}, \mathfrak{h}_{3} \oplus \mathfrak{h}_{3}, 3 \mathfrak{a f f} \mathfrak{R}_{\mathbb{R}}
$$

Remark 5.15 In [1], all six-dimensional Lie algebras with an Abelian complex structure have been classified. All these Lie algebra are two-step solvable since this is true in general for Lie algebras endowed with an Abelian complex structure. There are in total 8 (including the Abelian Lie algebra $\mathbb{R}^{6}$ ) six-dimensional Lie algebras and 12 single and 1 two-parameter family of six-dimensional non-nilpotent Lie algebras admitting an Abelian complex structure. By our results, 3 out of these 8 nilpotent and 4 out of the 12 single non-solvable admits Abelian complex structures coming from an SKT structure with totally real commutator ideal. Note that such an Abelian complex structure does not exist on any of the non-nilpotent Lie algebras in the two-parameter family.

\section{Commutator ideal complex of real-dimension two}

Here, we consider two-step SKT shear data $(\omega, \mathfrak{a})$ with $\mathfrak{a}$ being complex and of dimension two. Note that we then have $\mathfrak{a}=\mathfrak{a}_{J}, \mathfrak{a}_{r}=0, U_{r}=J \mathfrak{a}_{r}=0$ and so $U=U_{J}$.

We first show that $\left.\omega(Z, \cdot)\right|_{\mathfrak{a}}$ acts on $\mathfrak{a}$ by multiplication with an imaginary number. The proof will use a useful interpretation of the second part of Lemma 3.3(viii) in dimension 2. 
Lemma 6.1 Suppose $V=\mathbb{R}^{2}$, with the standard complex structure $J$. If $A_{1}, A_{2} \in \mathbb{R}^{2 \times 2}$ satisfy $(a)\left[A_{1}, A_{2}\right]=0$ and $(b)\left[A_{2}, J\right]=J\left[A_{1}, J\right]$, then both $A_{1}$ and $A_{2}$ commute with $J$.

Proof Decompose $A_{i}=A_{i}^{J}+A_{i}^{J-}$ with $J A_{i}^{J}=A_{i}^{J} J$ and $J A_{i}^{J-}=-A_{i}^{J-} J$. Then $\operatorname{dim}_{\mathbb{R}} V=2$, implies $\left[A_{1}^{J}, A_{2}^{J}\right]=0$, so (a) implies the $J$-anti-invariant parts $A_{i}^{J-}$ commute. These parts are symmetric matrices, so can be simultaneously diagonalised. However, (b) gives $2 A_{2}^{J-} J=\left[A_{2}, J\right]=J\left[A_{1}, J\right]=2 A_{1}^{J-}$, which for diagonal matrices implies $A_{i}^{J-}=0$.

Lemma 6.2 Let $(\omega, \mathfrak{a})$ be two-step SKT shear data with $\mathfrak{a}$ complex and of dimension two. Then there exists $\alpha \in U^{*}$ such that $\omega(Z, X)=\alpha(Z) J X$ for any $Z \in U, X \in \mathfrak{a}$.

Proof Fix $Z \in U$ of norm one and set $A_{1}:=\left.\omega(Z, \cdot)\right|_{\mathfrak{a}} \in \operatorname{End}(\mathfrak{a}), A_{2}:=\left.\omega(J Z, \cdot)\right|_{\mathfrak{a}} \in$ End(a). Inserting $\left(Z, J Z, X_{1}, X_{2}\right)$, for $X_{1}, X_{2} \in \mathfrak{a}$, into (3.2), we obtain

$$
\left.0=J A_{2}^{t} A_{2}+A_{2}^{t} A_{2} J+J A_{1}^{t} A_{1}+A_{1}^{t} A_{1} J+A_{1}^{t} J A_{1}^{J}+A_{1} J A_{1}+A_{2}^{t} J A_{2}^{J}+A_{2} J A 66.1\right)
$$

Equation (2.1) implies $\left[A_{1}, A_{2}\right]=0$ and Lemma 3.3(viii) gives $\left[A_{2}, J\right]=J\left[A_{1}, J\right]$, so by Lemma 6.1 both $A_{i}$ commute with $J$. Considering a as a complex one-dimensional space, we identify $A_{i}$ with $z_{i} \in \mathbb{C}$. As $J$ becomes multiplication by $i$ and transpose is complex conjugation, Eq. (6.1) is equivalent to

$$
0=\left|z_{1}\right|^{2}+\left|z_{2}\right|^{2}+z_{1}^{2}+z_{2}^{2}=\operatorname{Re}\left(z_{1}\right)^{2}+\operatorname{Re}\left(z_{2}\right)^{2},
$$

so $\operatorname{Re}\left(z_{1}\right)=0=\operatorname{Re}\left(z_{2}\right)$, giving the claimed statement.

Remark 6.3 Let $(\mathfrak{a}, \omega)$ be pre-shear data with $\mathfrak{a}$ being complex and of dimension two such that there is some $\alpha \in U^{*}$ with $\omega(Z, X)=\alpha(Z) J X$ for any $Z \in U, Y \in \mathfrak{a}$. Then (2.1) is fulfilled on $\mathfrak{a} \wedge \Lambda^{2} U$, (3.1) is satisfied on $\mathfrak{a} \wedge U$ and (3.2) is fulfilled on $\Lambda^{2} \mathfrak{a} \wedge \Lambda^{2} U$.

Next, we are going to show that if $\alpha \neq 0$, then $v_{i}=\lambda_{i} \alpha \wedge J^{*} \alpha$ for some $\lambda_{i} \in \mathbb{R}, i=1,2$, where $v_{1}, v_{2} \in \Lambda^{2} U^{*}$ are uniquely defined by $v=\sum_{i=1}^{2} v_{i} X_{i}$.

First of all, (2.1) is valid on $\Lambda^{3} U$ if and only if $\alpha \wedge v_{1}=0, \alpha \wedge v_{2}=0$. Next, (3.2) on $\mathfrak{a} \wedge \Lambda^{3} U$ gives us

$$
\begin{aligned}
& 0=J^{*} v_{2} \wedge \alpha+J^{*} \alpha \wedge v_{2}+\alpha \wedge v_{1}=J^{*} v_{2} \wedge \alpha+J^{*} \alpha \wedge v_{2} \\
& 0=J^{*} v_{1} \wedge \alpha+J^{*} \alpha \wedge v_{1}+\alpha \wedge v_{1}=J^{*} v_{1} \wedge \alpha+J^{*} \alpha \wedge v_{1}
\end{aligned}
$$

Now we may write $v_{i}=\alpha \wedge \beta_{i}$ for some $\beta_{i} \in U^{*}, i=1,2$, and then the just obtained equations give us $\alpha \wedge J^{*} \alpha \wedge \beta_{i}=0$ for all $i=1,2$. Hence, if $\alpha \neq 0$, then $\beta_{i} \in \operatorname{span}\left(\alpha, J^{*} \alpha\right)$, i.e. $v_{i}=\lambda_{i} \alpha \wedge J^{*} \alpha$ for some $\lambda_{i} \in \mathbb{R}, i=1$, 2. In this case, one easily checks that then (2.1)(3.2) are fulfilled and $\mathfrak{g} \cong \mathfrak{r}_{3,0}^{\prime} \oplus \mathbb{R}^{2 n-3}$.

Otherwise, $\alpha=0$ and so $\mathfrak{g}$ is nilpotent. Then (3.1) evaluated on $\Lambda^{2} U$ gives us $v_{1}=$ $\tau_{1}+\operatorname{Re}(\theta), v_{2}=\tau_{2}-\operatorname{Im}(\theta)$ for certain $\tau_{1}, \tau_{2} \in \Lambda^{1,1} U^{*}, \theta \in \Lambda^{2,0} U^{*}$. Then, we still have to solve (3.2) on $\Lambda^{4} U^{*}$, which gives us

$0=J^{*} v_{1} \wedge v_{1}+J^{*} v_{2} \wedge v_{2}=\left(\tau_{1}-\operatorname{Re}(\theta)\right) \wedge\left(\tau_{1}+\operatorname{Re}(\theta)\right)+\left(\tau_{2}-\operatorname{Im}(\theta)\right) \wedge\left(\tau_{2}+\operatorname{Im}(\theta)\right)$,

which is equivalent to

$$
\tau_{1} \wedge \tau_{1}+\tau_{2} \wedge \tau_{2}=\operatorname{Re}(\theta) \wedge \operatorname{Re}(\theta)+\operatorname{Im}(\theta) \wedge \operatorname{Im}(\theta)=\theta \wedge \bar{\theta} .
$$

We will now show the following 
Lemma 6.4 Let $(V, J)$ be a vector space with a complex structure. Suppose that $\omega \in \Lambda^{2,0} V$ and real $\sigma_{i} \in \Lambda^{1,1} V$ satisfy

$$
\sigma_{1}^{2}+\sigma_{2}^{2}=\omega \wedge \bar{\omega}
$$

Then the complex rank of $\omega$ is at most two and the common kernel of $\sigma_{1}, \sigma_{2}, \omega$ has codimension at most six.

Proof If $\omega=0$, the result follows from Lemma 5.9.

Otherwise, there is a decomposition $V=U \oplus W$ with $\operatorname{dim}(U)=4$ such that $\omega=$ $\alpha \wedge \beta+\omega^{\prime}$ with $\alpha, \beta \in \Lambda^{1,0} U^{*} \mathbb{C}$-linearly independent and $\omega^{\prime} \in \Lambda^{2,0} W^{*}$. We then have $\Lambda^{2} V^{*}=\Lambda^{2} U^{*}+U^{*} \wedge W^{*}+\Lambda^{2} W^{*}$ and correspondingly write elements of $\Lambda^{2} V^{*}$ as $\gamma=\gamma^{U}+\gamma^{m}+\gamma^{W}$.

We first show that $\omega^{\prime}=0$, i.e. that $\omega$ has complex rank two. For this, put $a=\operatorname{Re} \alpha$, $b=\operatorname{Re} \beta$. We may use the action of $\operatorname{SL}(2, \mathbb{C})$ as $\operatorname{SO}_{0}(1,3)$ on $\Lambda^{1,1}\left(\mathbb{C}^{2}\right)^{*}$ to get that $\sigma_{j}=$ $p(a \wedge J a+b \wedge J b)$ for some $p \in \mathbb{R}$ and then may use the remaining SU(2)-action on $\Lambda_{-}^{2} U^{*}$ to get

$$
\sigma_{2}^{U}=q a \wedge J a+r b \wedge J b,
$$

for some $q, r \in \mathbb{R}$. As $\left(\sigma_{1}^{U}\right)^{2}+\left(\sigma_{2}^{U}\right)^{2}=4 a \wedge J a \wedge b \wedge J b$, we get $p^{2}+q r=0$. Thus $(p, q) \neq(0,0)$ and we may rotate $\left(\sigma_{1}, \sigma_{2}\right)$ in such a way that $\sigma_{1}=p a \wedge J a+q b \wedge J b$ and $\sigma_{2}=r b \wedge J b$ for certain $p, q, r \in \mathbb{R}$ with $p>0$.

We may now write $\sigma_{j}^{m}=a \wedge x_{j}+J a \wedge J x_{j}+b \wedge y_{j}+J b \wedge J y_{j}$ for some $x_{j}, y_{j} \in W$. As $\omega \wedge \bar{\omega}$ has no component in $\Lambda^{3} U^{*} \wedge W^{*}$, we get $\sigma_{1}^{U} \wedge \sigma_{1}^{m}+\sigma_{2}^{U} \wedge \sigma_{2}^{m}=0$. The $a \wedge J a \wedge b$ term in this equation gives $p y_{1}=0$, so $y_{1}=0$.

Now the contribution to $\Lambda^{2} U^{*} \wedge \Lambda^{2} W^{*}$ is $\sum_{j=1}^{2}\left(2 \sigma_{j}^{U} \wedge \sigma_{j}^{W}+\left(\sigma_{j}^{m}\right)^{2}\right)=(a-i J a) \wedge$ $(b-i J b) \wedge \overline{\omega^{\prime}}+\omega^{\prime} \wedge(a+i J a) \wedge(b+i J b)$. Consider the terms multiplying $a \wedge b$. There is no contribution from $\sigma_{j}^{U}$ nor $\sigma_{1}^{m}$, so we get $-x_{2} \wedge y_{2}=\operatorname{Re}\left(\omega^{\prime}\right)$. Next, consider the terms multiplying $a \wedge b$. In this case, there is again no contribution from $\sigma_{j}^{U}$ and $\sigma_{1}^{m}$ and we have $x_{2} \wedge J y_{2}=\operatorname{Im}\left(\omega^{\prime}\right)$. So both $\operatorname{Re}\left(\omega^{\prime}\right)$ and $\operatorname{Im}\left(\omega^{\prime}\right)$ have rank two and, consequently, $\omega^{\prime} \wedge \overline{\omega^{\prime}}=0$. Thus $\omega^{\prime}=0$ and $\omega$ has rank two. Moreover, $x_{2}=0$ or $y_{2}=0$. We look again at the equation $\sigma_{1}^{U} \wedge \sigma_{1}^{m}+\sigma_{2}^{U} \wedge \sigma_{2}^{m}=0$. For $x_{2}=0$, the $b \wedge J b \wedge a$-term gives us $q x_{1}=0$ and so, since $q \neq 0$, that $x_{1}=0$ as well. For $y_{2}=0$, the same term gives us $q x_{1}+r x_{2}=0$, i.e. $x_{2}=-\frac{r}{q} x_{1}$.

Next, looking at the $\Lambda^{4} U^{*}$-terms, we have $\left(\sigma_{1}^{W}\right)^{2}+\left(\sigma_{2}^{W}\right)^{2}=0$ and so Lemma 5.9 shows the existence of $c, e \in W^{*}$ such that $\sigma_{1}^{W}=c \wedge J c$ and $\sigma_{2}^{W}=e \wedge J e$. Next, look at the $U^{*} \wedge \Lambda^{3} W^{*}$-terms, i.e. at the equation $\sigma_{1}^{m} \wedge \sigma_{1}^{W}+\sigma_{2}^{m} \wedge \sigma_{2}^{W}=0$.

If $x_{2}=0$, then we saw above that $\sigma_{1}^{m}=0$ and so we get $y_{1} \wedge e \wedge J e=0$, i.e. $y_{1}, e$ are $\mathbb{C}$-linearly dependent. Then the $a \wedge J a$-term in $\sum_{j=1}^{2}\left(2 \sigma_{j}^{U} \wedge \sigma_{j}^{W}+\left(\sigma_{j}^{m}\right)^{2}\right)=0$ gives us $p c \wedge J c=0$, i.e. $c=0$. Altogether, we see that the common kernel of $\sigma_{1}, \sigma_{2}$ in $W$ is contained in the kernel of $y_{1}, J y_{1}, e, J e$ and this kernel has codimension at most two since $y_{1}, e$ are $\mathbb{C}$-linearly dependent.

Finally, let $y_{2}=0$. Then $x_{2}=-\frac{r}{q} x_{1}$ and the equation $\sigma_{1}^{m} \wedge \sigma_{1}^{W}+\sigma_{2}^{m} \wedge \sigma_{2}^{W}=0$ gives us $x_{1} \wedge\left(c \wedge J c-\frac{r}{q} e \wedge J e\right)=0$. So either $x_{1}=0$ or $c, e$ are $\mathbb{C}$-linearly dependent. with $c \wedge J c=\frac{r}{q} e \wedge J e$.

In the first case $x_{2}=0$ as well, i.e. we have $\sigma_{1}^{m}=0, \sigma_{2}^{m}=0$, and we get again from $\sum_{j=1}^{2} 2 \sigma_{j}^{U} \wedge \sigma_{j}^{W}=\sum_{j=1}^{2}\left(2 \sigma_{j}^{U} \wedge \sigma_{j}^{W}+\left(\sigma_{j}^{m}\right)^{2}\right)=0$ that $c=0$ and the statement follows. Otherwise, $c, e$ are $\mathbb{C}$-linearly dependent with $c \wedge J c=\frac{r}{q} e \wedge J e$ and the equation 
$\sum_{j=1}^{2}\left(2 \sigma_{j}^{U} \wedge \sigma_{j}^{W}+\left(\sigma_{j}^{m}\right)^{2}\right)=0$ is equivalent to $p c \wedge J c-2\left(1+\frac{r^{2}}{q^{2}}\right) x_{1} \wedge J x_{1}=0$. This shows that the span of $c, J c, e, J e, x_{1}, J x_{1}$ is at most two-dimensional and the statement follows.

The last lemma implies that the $J$-invariant kernel of $\tau_{1}, \tau_{2} \in \Lambda^{1,1} U^{*}, \theta \in \Lambda^{2,0} U^{*}$ as above, i.e. satisfying

$$
\tau_{1} \wedge \tau_{1}+\tau_{2} \wedge \tau_{2}=\theta \wedge \bar{\theta}
$$

is of codimension at most 6 in $U$ and this kernel corresponds to an Abelian SKT Lie algebra. Thus, we have obtained:

Theorem 6.5 Let $(\mathfrak{g}, g, J)$ be an $2 n$-dimensional almost Hermitian Lie algebra such that $\mathfrak{g}^{\prime}$ is two-dimensional and $J$-invariant. Then $(\mathfrak{g}, g, J)$ is $S K T$ if and only if

(i) there exists $\alpha \in U^{*} \backslash\{0\}$ such that the only non-zero Lie brackets (up to anti-symmetry) are given by

$$
[Y, X]=\alpha(Y) J X
$$

for $X \in \mathfrak{g}^{\prime}, Y \in \mathfrak{g}^{\perp}$ or

(ii) there exists an orthonormal basis $(X, J X)$ of $\mathfrak{g}^{\prime}, \tau_{1}, \tau_{2} \in \Lambda^{1,1}\left(\mathfrak{g}^{\prime \perp}\right)^{*}$ and $\theta \in$ $\Lambda^{2,0}\left(\mathfrak{g}^{\prime \perp}\right)^{*}$ with $\tau_{1} \neq 0, \tau_{2} \neq 0$ or $\theta \neq 0$ and

$$
\tau_{1} \wedge \tau_{1}+\tau_{2} \wedge \tau_{2}=\theta \wedge \bar{\theta} .
$$

such that the only non-zero Lie brackets (up to anti-symmetry) are given by

$$
[Y, Z]=\left(\tau_{1}(Y, Z)+\operatorname{Re}(\theta)(Y, Z)\right) X+\left(\tau_{2}(Y, Z)-\operatorname{Im}(\theta)(Y, Z)\right) J X
$$

for $Y, Z \in \mathfrak{g}^{\perp}$.

Moreover, in case $(i)$, we have $\mathfrak{g} \cong \mathfrak{r}_{3,0}^{\prime} \oplus \mathbb{R}^{2 n-3}$, whereas in case (ii) $\mathfrak{g} \cong \mathfrak{h} \oplus \mathbb{R}^{2 n-2 k}$ for a $2 k$-dimensional two-step nilpotent Lie algebra $\mathfrak{h}$ with $k \in\{3,4\}$ and the splitting respects the SKT structure.

Remark 6.6 All algebras in Theorem 6.5 are unimodular; only those in case (ii) are nilpotent. All these cases may be explicitly described thanks to the classifications of nilpotent SKT algebras in dimensions 6 and 8 given in [11] and [7].

\section{Six-dimensional two-step solvable SKT Lie algebras}

In this section, we work towards classifying six-dimensional two-step solvable SKT Lie algebras $(\mathfrak{g}, g, J)$.

For this, let us first summarize what our previous results imply for this situation. First of all, Corollary 5.14 gives us a complete list in the cases when $\mathfrak{g}^{\prime}$ is totally real. Next, Theorem 6.5 describes all the cases with $\mathfrak{g}^{\prime}$ two-dimensional and complex, and Theorem 4.10, we determines cases with $\mathfrak{g}^{\prime}$ four-dimensional but non-complex. Finally, Corollary 4.8 contains a classification of the cases where $\mathfrak{g}^{\prime}$ has dimension 5 .

So, in order to classify all six-dimensional two-step solvable SKT Lie algebras, we still have to determine those with three-dimensional non-totally real $\mathfrak{g}^{\prime}$ and those with fourdimensional complex $\mathfrak{g}^{\prime}$. Unfortunately, it seems to be too hard to solve the latter case 
completely and we will just determine the relevant equations in Sect. 7.2 below. In contrast, the first case is treated completely in the next subsection and we note already now the following classification, that includes results from [11] for the nilpotent case and uses some of their notation.

Theorem 7.1 Let $(\mathfrak{g}, g, J)$ be a six-dimensional two-step solvable SKT Lie algebra. Then either $\mathfrak{g}$ is isomorphic as a Lie algebra to one of the following Lie algebras

$$
\begin{aligned}
& \mathbb{R}^{6}, \quad \mathfrak{a f f f} \\
& \mathfrak{R}_{\mathbb{R}} \oplus \mathbb{R}^{4}, \quad \mathfrak{a} \mathfrak{f} \mathfrak{f}_{\mathbb{R}} \oplus \mathfrak{h}_{3} \oplus \mathbb{R}, \quad 2 \mathfrak{a} \mathfrak{f f} \mathfrak{f}_{\mathbb{R}} \oplus \mathbb{R}^{2}, \quad 3 \mathfrak{a f f} \mathfrak{R}_{\mathbb{R}}, \quad \mathfrak{h}_{3} \oplus \mathbb{R}^{3}, \quad 2 \mathfrak{h}_{3}, \\
& \mathfrak{r}_{3,0}^{\prime} \oplus \mathbb{R}^{3}, \quad(0,0,0,0,12,14+23), \quad(0,0,0,0,13+42,14+23),
\end{aligned}
$$

or an algebra in Corollary 4.8 , or $(\mathfrak{g}, g, J)$ is isomorphic to one of the six-dimensional SKT Lie algebras in Theorem 4.10 or Theorem 7.5, or $\mathfrak{g}^{\prime}$ is four-dimensional, $J$-invariant, and, for a unit vector $X \in\left(\mathfrak{g}^{\prime}\right)^{\perp}$, the endomorphisms $A_{1}:=\left.\operatorname{ad}(X)\right|_{\mathfrak{g}^{\prime}}$ and $A_{2}:=\left.\operatorname{ad}(J X)\right|_{\mathfrak{g}^{\prime}}$ satisfy (7.10).

Remark 7.2 Note that Theorem 7.1 implies the four-dimensional two-step Lie algebras admitting an SKT structure are, in the notation of [18],

$$
\mathbb{R}^{4}, \quad \mathfrak{a} \mathfrak{f f} \mathfrak{R}_{\mathbb{R}} \oplus \mathbb{R}^{2}, \quad 2 \mathfrak{a} \mathfrak{f f}_{\mathbb{R}}, \quad \mathfrak{h}_{3} \oplus \mathbb{R}, \quad \mathfrak{r}_{3,0}^{\prime} \oplus \mathbb{R}, \quad \mathfrak{r}_{4, \lambda, 0}^{\prime}, \quad \mathfrak{r}_{4,-1 / 2,-1 / 2}, \quad \mathfrak{r}_{4,2 \lambda,-\lambda}^{\prime} .
$$

agreeing with the results of that paper.

\subsection{Three-dimensional commutator ideal not totally real}

Here, we consider two-step SKT shear data $(\mathfrak{a}, \omega)$ with $\operatorname{dim}(\mathfrak{a})=3$ and $\operatorname{dim}\left(\mathfrak{a}_{r}\right)=1$. Then $\mathfrak{a}_{J}$ and $U_{J}$ are real two-dimensional, and $U_{r}$ is real one-dimensional. Take orthonormal bases $(Y, J Y)$ of $\mathfrak{a}_{J}, X$ of $\mathfrak{a}_{r}$ and $(Z, J Z)$ of $U_{J}$. Then $J X$ is a basis of $U_{r}$ and $(Y, J Y, X)$ is an orthonormal basis of $\mathfrak{a}$. Setting $A:=-\omega_{0}(J X, \cdot) \in \operatorname{End}(\mathfrak{a}), B_{1}:=-\omega_{0}(Z, \cdot) \in \operatorname{End}(\mathfrak{a})$ and $B_{2}:=\omega_{0}(J Z, \cdot) \in \operatorname{End}(\mathfrak{a})$, Lemma 3.3(i) and (ii), give us with respect to the splitting $\mathfrak{a}=\mathfrak{a}_{J} \oplus \mathfrak{a}_{r}$

$$
A=\left(\begin{array}{ll}
z & u \\
0 & a
\end{array}\right)
$$

for some $a \in \mathbb{R}, z, u \in \mathbb{C}$. Replacing $X$ by $-X$ if necessary we may take $a \geq 0$. Equation (3.6) of Lemma 3.5, reduces to (4.6), and we conclude that $\operatorname{Re}(z) \in\{0,-a / 2\}$.

We now consider individually the cases $A=0$ and $A \neq 0$.

Lemma 7.3 With the above notation, if $A=0$, then the basis $(Z, J Z)$ of $U_{J}$ may be rotated so that $\omega(X, \cdot)=\omega(J X, \cdot)=0, \omega(Z, Y)=0, \omega(J Z, Y)=i b Y, b \in \mathbb{R}_{>0}$, and $\omega(Z, J Z)=$ $q Y+h X, h \in \mathbb{R} \backslash\{0\}$ and $q \in \mathbb{C}$.

Conversely, pre-shear data of this form is two-step SKT shear data with $\operatorname{im}(\omega)=\mathfrak{a}$ and the obtained Lie algebra is almost Abelian and isomorphic to $\mathfrak{g}_{5,14}^{0} \oplus \mathbb{R}$.

Proof Lemma 3.3(iv) and $a=0$ give $\omega(X, J X)=0$ and so

$$
\begin{aligned}
& 6 v_{1}(\omega)(X, J X, Z, J Z) \\
& \quad=-\|\omega(J X, J Z)\|^{2}-\|\omega(J X, Z)\|^{2}-\|\omega(X, J Z)\|^{2}-\|\omega(X, Z)\|^{2} .
\end{aligned}
$$

Moreover, in the current situation $\mathfrak{a} \perp U$, so we get

$$
12 v_{2}(\omega)(X, J X, Z, J Z)=g(\omega(J \omega(J X, Z), Z), X)+g(\omega(J \omega(J X, J Z), J Z), X) .
$$


Now $\omega^{r}(J \omega(J X, J Z), J Z)=\omega^{r}(\omega(J X, J Z), Z)$ by (3.1) since the other terms in this equation land in $U_{r}$. Moreover, $\omega^{r}(\omega(J X, J Z), Z)=\omega^{r}(\omega(J X, Z), J Z)$ by (2.1) as $\left.\omega(J X, \cdot)\right|_{\mathfrak{a}}=0$. Then, $\omega^{r}(\omega(J X, Z), J Z)=-\omega^{r}(J \omega(J X, Z), Z)$, again by (3.1). But so $g(\omega(J \omega(J X, J Z), J Z), X)=-g(\omega(J \omega(J X, Z), Z), X)$, which is equivalent to $\nu_{2}(\omega)(X, J X, Z, J Z)=0$. Thus, (3.2) implies (7.1) is zero, so

$$
\omega(X, Z)=\omega(J X, Z)=\omega(X, J Z)=\omega(J X, J Z)=0 .
$$

Hence, $\omega(X, \cdot)=\omega(J X, \cdot)=0$.

Next, $B_{i}=\left(\begin{array}{ll}C_{i} & 0 \\ y_{i}^{T} & 0\end{array}\right)$ with $C_{i} \in \mathbb{R}^{2 \times 2}$ and $y_{i} \in \mathbb{R}^{2}, i=1,2$. By (2.1), $\left[B_{1}, B_{2}\right]=0$, so $\left[C_{1}, C_{2}\right]=0$. Moreover, (3.1) gives us $\left[C_{2}, J\right]=J\left[C_{1}, J\right]$, Hence Lemma 6.1 implies that $C_{1}$ and $C_{2}$ commute with $J$. So write $c_{i} \in \mathbb{C}$ instead of $C_{i} \in \mathbb{R}^{2 \times 2}$ and consider also $y_{i}$ as complex numbers.

Then we observe that

$$
\begin{aligned}
6 & v_{1}(\omega)(Y, i Y, Z, J Z) \\
& =-\|\omega(i Y, J Z)\|^{2}-\|\omega(Y, J Z)\|^{2}-\|\omega(i Y, Z)\|^{2}-\|\omega(Y, Z)\|^{2} \\
& =-2\left|c_{1}\right|^{2}-2\left|c_{2}\right|^{2}-\left|y_{1}\right|^{2}-\left|y_{2}\right|^{2}
\end{aligned}
$$

and, using $\omega\left(J \omega\left(W_{1}, W_{2}\right), W_{3}\right)=0$ for all $W_{1}, W_{2} \in \mathbb{R}^{2 n}, W_{3} \in \mathfrak{a}_{J}$, we get

$$
\begin{aligned}
12 & \nu_{2}(\omega)(Y, i Y, Z, J Z) \\
= & -g(\omega(J \omega(Y, Z), Z), i Y)-g(\omega(J \omega(Y, J Z), J Z), i Y) \\
& +g(\omega(J \omega(i Y, J Z), J Z), Y)+g(\omega(J \omega(i Y, Z), Z), Y) \\
= & -g\left(C_{1} J C_{1} Y+C_{2} J C_{2} Y, i Y\right)+g\left(C_{2} J C_{2} i Y+C_{1} J C_{1} i Y, Y\right) \\
= & -2 g\left(\left(c_{1}^{2}+c_{2}^{2}\right) Y, Y\right)=-2 \operatorname{Re}\left(c_{1}^{2}\right)-2 \operatorname{Re}\left(c_{2}^{2}\right) .
\end{aligned}
$$

Since $\operatorname{Re}\left(c_{i}^{2}\right)+\left|c_{i}\right|^{2}=2 \operatorname{Re}\left(c_{i}\right)^{2}$ for $i=1,2$, Eq. (3.2) gives

$$
0=6\left(v_{1}(\omega)+2 v_{2}(\omega)\right)(Y, i Y, Z, J Z)=-4 \operatorname{Re}\left(c_{1}\right)^{2}-4 \operatorname{Re}\left(c_{2}\right)^{2}-\left|y_{1}\right|^{2}-\left|y_{2}\right|^{2} .
$$

Thus $\operatorname{Re}\left(c_{1}\right)=\operatorname{Re}\left(c_{2}\right)=y_{1}=y_{2}=0$. One may now check that $\omega$ satisfies all of the Eqs. (2.1), (3.1) and (3.2) and that $\operatorname{im}(\omega)=\mathfrak{a}$ precisely when $\left(c_{1}, c_{2}\right) \neq(0,0)$ and $b_{2} \neq 0$. Rotating $(Z, J Z)$ in such a way that $\omega(Z, Y)=0$, we may assume that $c_{1}=0$ and $c_{2}=i b$ for some $b \in \mathbb{R}_{>0}$. Writing $\omega(Z, J Z)=q Y+h X$ with $q \in \mathbb{C}$ and $h \in \mathbb{R}$, the condition $\operatorname{im} \omega=\mathfrak{a}$ gives $h \neq 0$ and we have the statement.

Next, we consider the case $A \neq 0$. For this, let us write

$$
\begin{aligned}
& \omega(J X, Z)=w_{1} Y+f_{1} X, \quad \omega(J X, J Z)=w_{2} Y+f_{2} X, \\
& \omega(Z, J Z)=q Y+h X,
\end{aligned}
$$

for some $w_{1}, w_{2}, q \in \mathbb{C}, f_{1}, f_{2}, h \in \mathbb{R}$.

Lemma 7.4 With the above notation, if $A=\left(\begin{array}{ll}z & u \\ 0 & a\end{array}\right) \neq 0$, then up to rotations in the $\operatorname{span}(Y, i Y)$ - and $\operatorname{span}(Z, J Z)$-planes, we have $u \in \mathbb{R}_{\geq 0}$ and either 
(i) $\operatorname{Re}(z)=-a / 2 \neq 0$,

$$
\begin{array}{ll}
B_{1}=\left(\begin{array}{cc}
i c_{1} & v_{1} \\
0 & 0
\end{array}\right), & B_{2}=\left(\begin{array}{cc}
-\frac{b_{2}}{2}+i c_{2} & v_{2} \\
0 & b_{2}
\end{array}\right) \\
v_{1}=-\frac{i c_{1}}{\frac{3}{2} a-i c} u, & v_{2}=\frac{\frac{3}{2} b_{2}-i c_{2}}{\frac{3}{2} a-i c} u,
\end{array}
$$

$z=-\frac{a}{2}+i c, f_{1}=-b_{2}, f_{2}=0, h=b_{2}^{2} / a$ and $w_{1}+i w_{2}=i\left(v_{1}+i v_{2}\right)$, or

(ii) $\operatorname{Re}(z)=0,(a, c=\operatorname{Im}(z)) \neq(0,0)$,

$$
\begin{array}{ll}
B_{1}=\left(\begin{array}{cc}
i c_{1} & v_{1} \\
0 & b_{1}
\end{array}\right), & B_{2}=\left(\begin{array}{cc}
i c_{2} & v_{2} \\
0 & b_{2}
\end{array}\right) \\
v_{1}=\frac{b_{1}-i c_{1}}{a-i c} u, & v_{2}=\frac{b_{2}-i c_{2}}{a-i c} u,
\end{array}
$$

$z=i c, f_{1}=-b_{2}, f_{2}=b_{1}$ and $w_{1}+i w_{2}=i\left(v_{1}+i v_{2}\right)$,

for some $b_{1}, b_{2}, c_{1}, c_{2}, c \in \mathbb{R}$.

Conversely, pre-shear data $(\mathfrak{a}, \omega)$ with $\operatorname{dim}(\mathfrak{a})=3$ and $\mathfrak{a}$ not totally real with all these properties satisfies (3.1) everywhere, (2.1) on $\Lambda^{2} U \wedge \mathfrak{a}$ and (3.2) on $\Lambda^{2} \mathfrak{a}_{J} \wedge \Lambda^{2} \mathbb{R}^{6}$.

Proof First we rotate the $\operatorname{span}(Y, i Y)$-plane to get $u \in \mathbb{R}_{\geq 0}$.

Note that $\operatorname{Re}(z) \in\{0,-a / 2\}$, implies either $z-a \neq 0$ or $z=0=a$. Consider $j \in\{1,2\}$. Since $A$ and $B_{j}$ commute, $B_{j}$ preserves the image and kernel of $A-a I_{3}$. As $\operatorname{span}\left(e_{1}, e_{2}\right)$ is one of these spaces, the image when $z-a \neq 0$, the kernel when $z=0=a$, this space is preserved by $B_{j}$. Thus,

$$
B_{j}=\left(\begin{array}{cc}
C_{j} & v_{j} \\
0 & b_{j}
\end{array}\right)
$$

with $C_{j} \in \mathbb{R}^{2 \times 2}, v_{j} \in \mathbb{R}^{2} \cong \mathbb{C}$ and $b_{j} \in \mathbb{R}$. Now $\left[B_{1}, B_{2}\right]=0$ gives $\left[C_{1}, C_{2}\right]=0$, furthermore Lemma 3.3(viii) means we can apply Lemma 6.1 to get that $C_{j}$ are $J$-invariant and may be identified with complex numbers. We may rotate $(Z, J Z)$ so that $\operatorname{Re}\left(C_{1}\right)=0$, i.e. $C_{1}=i c_{1}$ for some $c_{1} \in \mathbb{R}$.

Similarly to the proof of Lemma 7.3, we get

$$
6 v_{1}(\omega)(Y, i Y, Z, J Z)=-2\left|C_{1}\right|^{2}-2\left|C_{2}\right|^{2}
$$

and

$$
\begin{aligned}
12 v_{2}(\omega)(Y, i Y, Z, J Z)= & -2 \operatorname{Re}\left(C_{1}^{2}\right)-2 \operatorname{Re}\left(C_{2}^{2}\right) \\
& +g(\omega(J(\omega(Z, J Z)), i Y), i Y)+g(\omega(J(\omega(Z, J Z)), Y), Y) \\
= & -2\left(\operatorname{Re}\left(C_{1}^{2}\right)+\operatorname{Re}\left(C_{2}^{2}\right)-h \operatorname{Re}(z)\right) .
\end{aligned}
$$

Thus, (3.2) gives us

$$
\begin{aligned}
0 & =3 v_{1}(\omega)(Y, i Y, Z, J Z)+6 v_{2}(\omega)(Y, i Y, Z, J Z) \\
& =-2 \operatorname{Re}\left(C_{1}\right)^{2}-2 \operatorname{Re}\left(C_{2}\right)^{2}-h \operatorname{Re}(z)=-2 \operatorname{Re}\left(C_{2}\right)^{2}-h \operatorname{Re}(z),
\end{aligned}
$$

So $\operatorname{Re}\left(C_{2}\right)=0$ if $\operatorname{Re}(z)=0$, and $\operatorname{Re}\left(C_{2}\right)^{2}=a h / 4$ if $\operatorname{Re}(z)=-a / 2$.

Next,

$$
\begin{aligned}
6 v_{1}(\omega)(Y, i Y, X, Z) & =-g(\omega(i Y, J X), \omega(i Y, Z))-g(\omega(Y, J X), \omega(Y, Z)) \\
& =-2 \operatorname{Re}\left(z \bar{C}_{1}\right)
\end{aligned}
$$


and

$$
\begin{aligned}
12 v_{2}(\omega)(Y, i Y, X, Z) & =-g(\omega(J \omega(Y, Z), J X), i Y)+g(\omega(J \omega(i Y, Z), J X), Y) \\
& +g(\omega(J \omega(X, Z), i Y), i Y)+g(\omega(J \omega(X, Z), Y), Y) \\
& =-2 \operatorname{Re}\left(z C_{1}\right)-2 b_{1} \operatorname{Re}(z),
\end{aligned}
$$

so that (3.2) gives us

$$
\begin{aligned}
0 & =6 v(\omega)(Y, i Y, X, Z)=-2 \operatorname{Re}\left(z \bar{C}_{1}\right)-2 \operatorname{Re}\left(z C_{1}\right)-2 b_{1} \operatorname{Re}(z) \\
& =-2 \operatorname{Re}(z)\left(2 \operatorname{Re}\left(C_{1}\right)+b_{1}\right)=-2 \operatorname{Re}(z) b_{1} .
\end{aligned}
$$

Hence, if $\operatorname{Re}(z)=-a / 2 \neq 0$, then $b_{1}=0$. Similarly, we obtain

$$
0=6 v(\omega)(Y, i Y, X, J Z)=-2 \operatorname{Re}(z)\left(2 \operatorname{Re}\left(C_{2}\right)+b_{2}\right),
$$

and so $\operatorname{Re}\left(C_{2}\right)=-b_{2} / 2$ if $\operatorname{Re}(z)=-a / 2 \neq 0$, which implies $b_{2}^{2} / 4=\operatorname{Re}\left(C_{2}\right)^{2}=a h / 4$ and hence $h=b_{2}^{2} / a$.

Note that $v_{1}(\omega)(Y, i Y, J X, Z)=v_{1}(\omega)(i Y, Y, X, J Z)=-v_{1}(\omega)(Y, i Y, X, J Z)$ and that

$$
\begin{aligned}
v_{2}(\omega)(Y, i Y, J X, Z)= & g(\omega(J \omega(Y, J X), J Z), i Y)-g(\omega(J \omega(i Y, J X), J Z), Y) \\
& +g(\omega(J \omega(J X, Z), i Y), i Y)+g(\omega(J \omega(J X, Z), Y), Y) \\
= & g(i \omega(\omega(Y, J X), J Z), i Y)-g(i \omega(\omega(i Y, J X), J Z), Y) \\
& +g\left(\omega\left(J \omega^{r}(J X, Z), i Y\right), i Y\right)+g\left(\omega\left(J \omega^{r}(J X, Z), Y\right), Y\right) \\
= & g(\omega(J \omega(Y, J Z), J X), i Y)-g(\omega(J \omega(i Y, J Z), J X), Y) \\
& -g(\omega(J \omega(X, J Z), i Y), i Y)-g(\omega(J \omega(X, J Z), Y), Y) \\
= & -v_{2}(\omega)(Y, i Y, X, J Z),
\end{aligned}
$$

so that $v(\omega)(Y, i Y, J X, Z)=-v(\omega)(Y, i Y, X, J Z)=0$. Similarly, the identity $v(\omega)(Y, i Y, J X, J Z)=v(\omega)(Y, i Y, X, Z)=0$ follows. Thus, (3.2) is also valid on $\Lambda^{2} \mathfrak{a}_{J} \wedge U_{r} \wedge U_{J}$, and so on $\Lambda^{2} \mathfrak{a}_{J} \wedge \Lambda^{2} \mathbb{R}^{6}$.

Next, we notice that (3.1) is satisfied if and only if parts (vi) and (vii) of Lemma 3.3 hold. Now (vi) is equivalent to $f_{1}=-b_{2}$ and $f_{2}=b_{1}$ and (vii) is equivalent to

$$
w_{1}+i w_{2}=i\left(v_{1}+i v_{2}\right) \text {. }
$$

Moreover, by (2.1), we must have $\left[A, B_{i}\right]=0$, which is equivalent to $z v_{i}+u b_{i}=C_{i} u+v_{i} a$, i.e. to $(z-a) v_{i}=\left(C_{i}-b_{i}\right) u$, and so to $v_{i}=\frac{C_{i}-b_{i}}{z-a} u, i=1,2$, if $z-a \neq 0$.

If $z-a=0$, then $z=0=a$ and $u \neq 0$. As $C_{j}=i c_{j}$, we have $0=(z-a) v_{j}=\left(i c_{j}-b_{j}\right) u$, giving $b_{j}=c_{j}=0$ for $j=1,2$. This implies $\omega(X, Z), \omega(J X, Z), \omega(X, J Z), \omega(J X, J Z)$ all lie in $\mathfrak{a}_{J}$ and $\mathfrak{a}_{J} \subseteq \operatorname{ker}(\omega(J X, \cdot)) \cap \operatorname{ker}(\omega(Z, \cdot)) \cap \operatorname{ker}(\omega(J Z, \cdot))$. Using these properties, (2.1) implies

$$
\begin{aligned}
0 & =\omega(J X, \omega(Z, J Z))+\omega(Z, \omega(J Z, J X))+\omega(J Z, \omega(J X, Z)) \\
& =\omega(J X, q Y+h X)=u h Y .
\end{aligned}
$$

Since $u \neq 0$, we get $h=0$ and thus $\operatorname{im}(\omega) \subseteq \mathfrak{a}_{J}$, a contradiction. We conclude that $z-a \neq 0$, which is clear if $\operatorname{Re}(z)=-a / 2 \neq 0$ but implies $a \neq 0$ or $\operatorname{Im}(z) \neq 0$ in the case $\operatorname{Re}(z)=0$.

Summarizing our results to this point, we observe that we still have to impose (2.1) on $\Lambda^{3} U=U_{r} \wedge \Lambda^{2} U_{J}$ and (3.2) on $\mathfrak{a}_{J} \wedge \Lambda^{3}\left(\mathfrak{a}_{r} \oplus U_{r} \oplus U_{J}\right)$ and on $\Lambda^{4}\left(\mathfrak{a}_{r} \oplus U_{r} \oplus U_{J}\right)=$ $\mathfrak{a}_{r} \wedge U_{r} \wedge \Lambda^{2} U_{J}$ 
First of all, (2.1) evaluated on $U_{r} \wedge \Lambda^{2} U_{J}$ yields

$$
\begin{aligned}
0 & =\omega(J X, \omega(Z, J Z))+\omega(Z, \omega(J Z, J X))+\omega(J Z, \omega(J X, Z)) \\
& =A \cdot(q, h)^{T}-B_{1}\left(w_{2}, b_{1}\right)^{T}+B_{2}\left(w_{1},-b_{2}\right),
\end{aligned}
$$

where we set $b_{1}=0$ if $\operatorname{Re}(z)=-a / 2$. This equation is equivalent to

$$
0=z q+h u-i c_{1} w_{2}-b_{1} v_{1}+C_{2} w_{1}-b_{2} v_{2} \text { and } a h=\|b\|^{2}
$$

Note that for $\operatorname{Re}(z)=-a / 2 \neq 0$, the second equation in automatically satisfied as $b_{1}=0$ and $h=b_{2}^{2} / a$.

Let us now first look at (3.2) on $\mathfrak{a}_{J} \wedge \mathfrak{a}_{r} \wedge U_{r} \wedge U_{J}$. For this, let $\tilde{Y} \in \mathfrak{a}_{J}$. Then

$$
\begin{aligned}
6 v_{1}(\omega)(\tilde{Y}, X, J X, Z)= & g(\omega(i \tilde{Y}, J X), \omega(J X, Z))+g(\omega(i \tilde{Y}, J Z), \omega(X, J X)) \\
& -g(\omega(J X, X), \omega(\tilde{Y}, Z))-g(\omega(J X, J Z), \omega(\tilde{Y}, J X)) \\
= & g\left(i z \tilde{Y}, w_{1} Y\right)+g\left(i C_{2} \tilde{Y}, u Y\right)+g\left(u Y, i c_{1} \tilde{Y}\right)-g\left(w_{2} Y, z \tilde{Y}\right)
\end{aligned}
$$

and

$$
\begin{aligned}
12 v_{2}(\omega)(\tilde{Y}, X, J X, Z)= & -g(\omega(J \omega(X, J X), J Z), \tilde{Y})-g(\omega(J \omega(J X, Z), J X), \tilde{Y}) \\
& +g(\omega(J \omega(Z, X), X), \tilde{Y}) \\
= & -g\left(i u C_{2} Y, \tilde{Y}\right)-g\left(a w_{2} Y, \tilde{Y}\right)-g\left(i w_{1} z Y, \tilde{Y}\right)+g\left(b_{1} u Y, \tilde{Y}\right) .
\end{aligned}
$$

Thus, $v(\omega)(\tilde{Y}, X, J X, Z)=0$ for all $\tilde{Y} \in \mathfrak{a}_{J}$ is equivalent to

$$
0=-2 i \operatorname{Re}(z) w_{1}-2 i \operatorname{Re}\left(C_{2}\right) u-i c_{1} u-w_{2} \bar{z}-a w_{2}+b_{1} u .
$$

Similarly, $v(\omega)(\tilde{Y}, X, J X, J Z)=0$ for all $\tilde{Y} \in \mathfrak{a}_{J}$ is seen to be equivalent to

$$
\begin{aligned}
0 & =-i \bar{z} w_{2}+c_{1} u+\bar{C}_{2} u+\bar{z} w_{1}-u c_{1}+a w_{1}-i w_{2} z+b_{2} u \\
& =-2 i \operatorname{Re}(z) w_{2}+\bar{C}_{2} u+w_{1} \bar{z}+a w_{1}+b_{2} u .
\end{aligned}
$$

Analogous computations give $v(\omega)(\widetilde{Y}, X, Z, J Z)=0$ for all $\widetilde{Y} \in \mathfrak{a}_{J}$ if and only if

$$
0=2 i v_{2} \operatorname{Re}\left(C_{2}\right)-2 i q \operatorname{Re}(z)+b_{1} w_{1}+b_{2} w_{2}+\bar{C}_{2} w_{2}-i c_{1} w_{1}
$$

and that $v(\omega)(\tilde{Y}, J X, Z, J Z)=0$ for all $\tilde{Y} \in \mathfrak{a}_{J}$ if and only if

$$
0=2 i w_{2} \operatorname{Re}\left(C_{2}\right)-\bar{C}_{2} v_{2}+i c_{1} v_{1}+q \bar{z}-b_{2} w_{1}-h u+b_{1} w_{2}
$$

Finally, we have to consider the equation $v(\omega)(X, J X, Z, J Z)=0$. Using

$$
\begin{aligned}
\omega^{r}(J \omega(J X, J Z), J Z) & =\omega^{r}(\omega(J X, J Z), Z) \\
& =\omega^{r}(\omega(Z, J Z), J X)+\omega^{r}(\omega(J X, Z), J Z) \\
& =-\omega^{r}(J \omega(Z, J Z), X)-\omega^{r}(J \omega(J X, Z), Z),
\end{aligned}
$$

one easily sees that

$$
\nu_{2}(\omega)(X, J X, Z, J Z)=0 .
$$

Thus, $0=v(\omega)(X, J X, Z, J Z)=v_{1}(\omega)(X, J X, Z, J Z)$, and a short computation yields that this equation is equivalent to

$$
\begin{aligned}
0= & 2 g(\omega(X, J X), \omega(Z, J Z))-\|\omega(X, Z)\|^{2}-\|\omega(X, J Z)\|^{2} \\
& -\|\omega(J X, Z)\|^{2}-\|\omega(J X, J Z)\|^{2} .
\end{aligned}
$$


Let us now consider individually the cases (a) $\operatorname{Re}(z)=0$ and (b) $\operatorname{Re}(z)=-a / 2 \neq 0$.

(a) $\operatorname{Re}(z)=0$. Here, $\operatorname{Re}\left(C_{2}\right)=0$ and so $C_{2}=i c_{2}$ for some $c_{2} \in \mathbb{R}$. Then (7.4) and (7.5) imply

$$
w_{2}=\frac{b_{1}-i c_{1}}{a-i c} u=v_{1}, \quad w_{1}=-\frac{b_{2}-i c_{2}}{a-i c} u=-v_{2},
$$

which is consistent with $w_{i}+i w_{2}=i\left(v_{1}+i v_{2}\right)$. Moreover, (7.6) is equivalent to $\left(b_{2}-\right.$ $\left.i c_{2}\right) w_{2}=-\left(b_{1}-i c_{1}\right) w_{1}$, and so also automatically satisfied. Furthermore, the first equation in (7.3) and (7.7) are both easily seen to be equivalent to

$$
\begin{aligned}
0 & =i c q+h u-\left(b_{1}+i c_{1}\right) v_{1}-\left(b_{2}+i c_{2}\right) v_{2}=i c q+h u-\frac{b_{1}^{2}+b_{2}^{2}+c_{1}^{2}+c_{2}^{2}}{a-i c} u \\
& =i c q+\frac{h a-i h c-b_{1}^{2}-b_{2}^{2}-c_{1}^{2}-c_{2}^{2}}{a-i c} u=i c q-\frac{i h c+c_{1}^{2}+c_{2}^{2}}{a-i c} u .
\end{aligned}
$$

So if $c \neq 0$, we obtain

$$
q=\frac{i c h+c_{1}^{2}+c_{2}^{2}}{c^{2}+a c i} u
$$

If $c=0$, we would get $c_{1}^{2}+c_{2}^{2}=0$, hence $c_{1}=c_{2}=0$. However, this case cannot occur as then the only non-zero Lie brackets on the shear would be (up to anti-symmetry) $[Z, J Z]$ and

$$
[X, J X]=\frac{b_{1}}{a}[X, Z]=\frac{b_{1}}{a}[J X, J Z]=\frac{b_{2}}{a}[X, J Z]=-\frac{b_{2}}{a}[J X, Z]
$$

giving $\operatorname{dim}\left(\mathfrak{g}^{\prime}\right) \leq 2<3$, a contradiction.

Thus, $c \neq 0$ and we still have to satisfy (7.8). Due to $\omega(J X, J Z)=\omega(X, Z)$ and $\omega(J X, Z)=-\omega(X, J Z)$ and $h a=\|b\|^{2}$, this equation is

$$
0=2 \operatorname{Re}(q) u+2 h a-2\left|v_{1}\right|^{2}-2 b_{1}^{2}-2\left|v_{2}\right|^{2}-2 b_{2}^{2}=2\left(\operatorname{Re}(q) u-\left|v_{1}\right|^{2}-\left|v_{2}\right|^{2}\right),
$$

so

$$
\operatorname{Re}(q) u=\left|v_{1}\right|^{2}+\left|v_{2}\right|^{2}=\frac{b_{1}^{2}+b_{2}^{2}+c_{1}^{2}+c_{2}^{2}}{a^{2}+c^{2}} u^{2} .
$$

On the other hand, $q=\frac{i c h+c_{1}^{2}+c_{2}^{2}}{c^{2}+a c i} u$ implies

$$
\operatorname{Re}(q) u=\operatorname{Re}\left(\frac{i c h+c_{1}^{2}+c_{2}^{2}}{c^{2}+a c i} u\right) u=\frac{c_{1}^{2}+c_{2}^{2}+a h}{a^{2}+c^{2}} u^{2}=\frac{c_{1}^{2}+c_{2}^{2}+b_{1}^{2}+b_{2}^{2}}{a^{2}+c^{2}} u^{2},
$$

and so (7.8) is automatically satisfied.

(b) $\operatorname{Re}(z)=-a / 2 \neq 0$. In this case, (7.4) and (7.5) are explicitly given by

$$
i a w_{1}-\left(\frac{a}{2}-i c\right) w_{2}+i\left(b_{2}-c_{1}\right) u=0, \quad i a w_{2}+\left(\frac{a}{2}-i c\right) w_{1}+\left(\frac{b_{2}}{2}-i c_{2}\right) u=0 .
$$

The solution is given by

$$
\begin{aligned}
& w_{1}=-\frac{\left(\frac{a}{2}-i c\right)\left(\frac{b_{2}}{2}-i c_{2}\right)-a\left(b_{2}-c_{1}\right)}{\left(\frac{a}{2}-i c\right)^{2}-a^{2}} u, \\
& w_{2}=i \frac{\left(\frac{a}{2}-i c\right)\left(b_{2}-c_{1}\right)-a\left(\frac{b_{2}}{2}-i c_{2}\right)}{\left(\frac{a}{2}-i c\right)^{2}-a^{2}} u,
\end{aligned}
$$

and $w_{1}+i w_{2}=i\left(v_{1}+i v_{2}\right)$ holds. 
Next, using $w_{2}=v_{1}+i v_{2}+i w_{1},(7.7)$ is explicitly given by

$$
\begin{aligned}
0 & =-i b_{2} w_{2}+\left(\frac{b_{2}}{2}+i c_{2}\right) v_{2}+i c_{1} v_{1}-\left(\frac{a}{2}+i c\right) q-b_{2} w_{1}-h u \\
& =-i b_{2} v_{1}+b_{2} v_{2}+\left(\frac{b_{2}}{2}+i c_{2}\right) v_{2}+i c_{1} v_{1}-\left(\frac{a}{2}+i c\right) q-h u \\
& =\left(\frac{3}{2} b_{2}+i c_{2}\right) v_{2}-i\left(b_{2}-c_{1}\right) v_{1}-\left(\frac{a}{2}+i c\right) q-h u,
\end{aligned}
$$

and so we do get

$$
q=\frac{-i\left(b_{2}-c_{1}\right) v_{1}+\left(\frac{3}{2} b_{2}+i c_{2}\right) v_{2}-h u}{\frac{a}{2}+i c}=\frac{\left(c_{1}-b_{2}\right) c_{1}+\frac{9}{4} b_{2}^{2}+c_{2}^{2}-h\left(\frac{3}{2} a-i c\right)}{\left(\frac{a}{2}+i c\right)\left(\frac{3}{2} a-i c\right)} u .
$$

A lengthy but straightforward computation shows that then the first equation in (7.3) and also (7.6) and (7.8) are satisfied.

Theorem 7.5 Let $(\mathfrak{g}, g, J)$ be a six-dimensional almost Hermitian Lie algebra. Then $(\mathfrak{g}, g, J)$ is a two-step solvable SKT Lie algebra with $\mathfrak{g}^{\prime}$ three-dimensional and not totally real if and only if $(\mathfrak{g}, g, J)$ admits a unitary $(Y, i Y, X, J X, Z, J Z)$ basis such that $Y$ spans $\mathfrak{g}_{J}^{\prime}$ as a complex vector space and $X$ spans the real vector space $\mathfrak{g}_{r}^{\prime}$ and the only non-zero Lie brackets (up to anti-symmetry and complex-linear extension to $\mathfrak{g}_{J}^{\prime}$ ) are given by either

(i) $[J Z, Y]=i b Y,[Z, J Z]=q Y+h X$ for $b, h \in \mathbb{R} \backslash\{0\}, q \in \mathbb{C}$,

(ii)

$$
=i c Y, \quad[Z, Y]=i c_{1} Y, \quad[J Z, Y]=i c_{2} Y,
$$

$[J X, X]=u Y+a X, \quad[Z, X]=[J Z, J X]=-\frac{i c_{1}}{\frac{3}{2} a-i c} u Y+b_{1} X$,

$[J Z, X]=-[Z, J X]=\frac{\frac{3}{2} b_{2}-i c_{2}}{\frac{3}{2} a-i c} u Y+b_{2} X, \quad[Z, J Z]=-\frac{i c h+c_{1}^{2}+c_{2}^{2}}{c^{2}+a c i} u Y-h X$

for certain $c \in \mathbb{R} \backslash\{0\}, b_{1}, b_{2}, c_{1}, c_{2}, h \in \mathbb{R}, a, u \in \mathbb{R}_{\geq 0}$ with $h a=b_{1}^{2}+b_{2}^{2}$ and at least one of $a, b_{1}, b_{2}, h$ non-zero, or

(iii)

$$
\begin{aligned}
& =\left(-\frac{a}{2}+i c\right) Y, \quad[Z, Y]=i c_{1} Y, \quad[J Z, Y]=\left(-\frac{b_{2}}{2}+i c_{2}\right) Y, \\
{[J X, X] } & =u Y+a X, \quad[Z, X]=-\frac{i c_{1}}{\frac{3}{2} a-i c} u Y, \\
{[J Z, X] } & =\frac{\frac{3}{2} b_{2}-i c_{2}}{\frac{3}{2} a-i c} u Y+b_{2} X, \quad[Z, J Z]=-q Y-h X, \\
{[Z, J X] } & =-\frac{\left(\frac{a}{2}-i c\right)\left(\frac{b_{2}}{2}-i c_{2}\right)-a\left(b_{2}-c_{1}\right)}{\left(\frac{a}{2}-i c\right)^{2}-a^{2}} u Y-b_{2} X, \\
{[J Z, J X] } & =i \frac{\left(\frac{a}{2}-i c\right)\left(b_{2}-c_{1}\right)-a\left(\frac{b_{2}}{2}-i c_{2}\right)}{\left(\frac{a}{2}-i c\right)^{2}-a^{2}} u Y
\end{aligned}
$$

for $a \in \mathbb{R} \backslash\{0\}, b_{2}, c, c_{1}, c_{2} \in \mathbb{R}, u \in \mathbb{R}_{\geq 0}$, with

$$
h=\frac{b_{2}^{2}}{a}, \quad q=\frac{\left(c_{1}-b_{2}\right) c_{1}+\frac{9}{4} b_{2}^{2}+c_{2}^{2}-h\left(\frac{3}{2} a-i c\right)}{\left(\frac{a}{2}+i c\right)\left(\frac{3}{2} a-i c\right)} u .
$$


Remark 7.6 Identifying the unimodular Lie algebras in Theorem 7.5, one observes that those in (i) and (iii) are always unimodular whereas those in (ii) are unimodular precisely when $a=b_{1}=b_{2}=0$ (and then $h \neq 0$ ). Note that none of the Lie algebras in Theorem 7.5 can be nilpotent.

\subsection{Four-dimensional complex commutator ideal}

As announced at the beginning of Sect. 7, we only write down the equations in this case.

First of all, note that $\mathfrak{a}=\mathfrak{a}_{J}, U=U_{J}$ and take an orthonormal basis $Y_{1}, Y_{2}=J Y_{1}$ of $U$. Then $\omega$ is completely determined by $A_{i}:=\omega_{0}\left(Y_{i}, \cdot\right) \in \operatorname{End}(\mathfrak{a}), i=1,2$, and by $X:=\omega_{1}\left(Y_{1}, Y_{2}\right) \in \mathfrak{a}$. Moreover, $(2.1)$ reduces to $\left[A_{1}, A_{2}\right]=0$ and $(3.1)$ reduces to $\left[A_{2}, J\right]=$ $J\left[A_{1}, J\right]$, i.e. $A_{2}^{J-}=J A_{1}^{J-}$, where the superscript $J-$ denotes the $J$-anti-invariant part of an endomorphism of $\mathfrak{a}=\mathfrak{a}_{J}$. Finally, (3.2) reduces to

$$
J A_{1}^{T} A_{1}+A_{1}^{T} A_{1} J+J A_{2}^{T} A_{2}+A_{2}^{T} A_{2} J+A_{1} J A_{1}+A_{1}^{T} J A_{1}^{T}+A_{2} J A_{2}+A_{2}^{T} J A_{2}^{T}=0 .
$$

where the transpose is taken with respect to the Riemannian metric $\left.g\right|_{\mathfrak{a}}$ on $\mathfrak{a}$. Note that this is equivalent to $J A_{1}^{T} A_{1}+A_{1} J A_{1}+J A_{2}^{T} A_{2}+A_{2} J A_{2}$ being symmetric. Summarizing, $A_{1}$ and $A_{2}$ have to satisfy the following equations:

$$
\begin{aligned}
& {\left[A_{1}, A_{2}\right]=0, \quad A_{2}^{J-}=J A_{1}^{J-},} \\
& \sum_{i=1}^{2} J A_{i}^{T} A_{i}+A_{i}^{T} A_{i} J+A_{i} J A_{i}+A_{i}^{T} J A_{i}^{T}=0 .
\end{aligned}
$$

Writing $A_{i}=\left(A_{i}\right)_{+}+\left(A_{i}\right)_{-}$, with $\left(A_{i}\right)_{\varepsilon}^{T}=\varepsilon\left(A_{i}\right)_{\varepsilon}$, for $\varepsilon \in\{ \pm 1\}$, this decomposition commutes with the decomposition $A_{i}=A_{i}^{J}+A_{i}^{J-}$ and the final equation in (7.10) become the two equations

$$
\begin{aligned}
& \sum_{i=1}^{2}\left[\left(A_{i}\right)_{+}^{J},\left(A_{i}\right)_{+}^{J-}\right]-\left[\left(A_{i}\right)_{-}^{J-},\left(A_{i}\right)_{-}^{J}\right]=0, \\
& \sum_{i=1}^{2}\left[\left(A_{i}\right)_{+}^{J},\left(A_{i}\right)_{-}^{J-}\right]-\left[\left(A_{i}\right)_{-}^{J-},\left(A_{i}\right)_{+}^{J}\right]+2\left(\left(A_{i}\right)_{+}^{J}\right)^{2}-2\left(\left(A_{i}\right)_{-}^{J-}\right)^{2}=0 .
\end{aligned}
$$

Acknowledgements The first author was partly supported by a Forschungsstipendium (FR 3473/2-1) from the Deutsche Forschungsgemeinschaft (DFG).

Funding Open Access funding enabled and organized by Projekt DEAL.

Open Access This article is licensed under a Creative Commons Attribution 4.0 International License, which permits use, sharing, adaptation, distribution and reproduction in any medium or format, as long as you give appropriate credit to the original author(s) and the source, provide a link to the Creative Commons licence, and indicate if changes were made. The images or other third party material in this article are included in the article's Creative Commons licence, unless indicated otherwise in a credit line to the material. If material is not included in the article's Creative Commons licence and your intended use is not permitted by statutory regulation or exceeds the permitted use, you will need to obtain permission directly from the copyright holder. To view a copy of this licence, visit http://creativecommons.org/licenses/by/4.0/. 
Table 1 Notation for certain Lie algebras

\begin{tabular}{llll}
\hline $\mathfrak{g}$ & $\operatorname{dim}$ & Differentials & Conditions \\
\hline $\mathfrak{r}_{3, \lambda}^{\prime}$ & 3 & $(0, \lambda .21+31,-21+\lambda .31)$ & $\lambda \geq 0$ \\
$\mathfrak{r}_{4, \mu, \lambda}$ & 4 & $(0,21, \mu .31, \lambda .41)$ & $0<|\lambda| \leq|\mu| \leq 1$ \\
$\mathfrak{r}_{4, \mu, \lambda}^{\prime}$ & 4 & $(0, \mu .21, \lambda .31+41,-31+\lambda .41)$ & $\mu>0$ \\
$\mathfrak{g}_{5,14}^{\alpha}$ & 5 & $(0,0,21, \alpha .41+51,-41+\alpha .51)$ & $\alpha \geq 0$ \\
$\mathfrak{g}_{5,17}^{\alpha, \beta, \gamma}$ & 5 & $(0, \alpha .21+31,-21+\alpha .31$, & $\alpha \geq 0, \gamma \neq 0$ \\
$\mathfrak{g}_{6,1}^{\alpha, \beta, \gamma, \delta}$ & 6 & $(0,21, \alpha .31, \beta .41, \gamma \cdot 51, \delta .61)$ & $0<|\delta| \leq|\gamma| \leq|\beta| \leq|\alpha| \leq 1$ \\
$\mathfrak{g}_{6,8}^{\alpha, \beta, \gamma, \delta}$ & 6 & $(0, \alpha .21, \beta .31, \gamma .41$, & $0<|\gamma| \leq|\beta| \leq|\alpha|$ \\
$\mathfrak{g}_{6,11}^{\alpha, \beta, \gamma, \delta}$ & 6 & $\delta .51+61,-51+\delta .61)$ & \\
$(37 D)$ & 7 & $(0, \alpha .21, \beta .31+41,-31+\beta .41$, & \\
\hline
\end{tabular}

\section{Appendix A: Notation for particular Lie algebras}

Table 1 contains a list of all indecomposable Lie algebras $\mathfrak{g}$ of various dimensions which occur in this article and which are not well-known, in contrast to the Abelian Lie algebra $\mathbb{R}$, the two-dimensional Lie algebra $\mathfrak{a f f} \mathfrak{R}_{\mathbb{R}}$ of affine motions of the real line and the $(2 k+1)$ dimensional Heisenberg Lie algebra $\mathfrak{h}_{2 k+1}$. The names for Lie algebras in dimension 3 are taken from [5], in four dimensions from [2], in five dimensions from [19], in six dimensions from [20] and in seven dimensions from [16].

In all cases, the Lie bracket is encoded dually by the tuple of differentials $\left(d e^{1}, \ldots, d e^{n}\right)$ for a basis $e^{1}, \ldots, e^{n}$ of $\mathfrak{g}$ and we use Salamon's notation for this tuple. Moreover, in some cases, we do not give all the necessary conditions on the parameters to ensure that two Lie algebras in the corresponding parameter families are non-isomorphic.

\section{References}

1. Andrada, A., Barberis, M.L., Dotti, I.: Classification of abelian complex structures on 6-dimensional Lie algebras. J. Lond. Math. Soc. (2) 83(1), 232-255 (2011)

2. Andrada, A., Barberis, M.L., Dotti, I.G., Ovando, G.P.: Product structures on four dimensional solvable Lie algebras. Homology Homotopy Appl. 7(1), 9-37 (2005)

3. Apostolov, V., Gualtieri, M.: Generalized Kähler manifolds, commuting complex structures, and split tangent bundles. Comm. Math. Phys. 271(2), 561-575 (2007)

4. Arroyo, R.M., Lafuente, R.A.: The long-time behavior of the homogeneous pluriclosed flow. Proc. Lond. Math. Soc. (3) 119(1), 266-289 (2019)

5. Bianchi, L.: Sugli spazi a tre dimensioni che ammettono un gruppo continuo di movimenti. Mem. Mat. Fis. Ital. Sci. Serie Terza 11, 267-352 (1898)

6. Cavalcanti, G.R.: Reduction of metric structures on Courant algebroids. J. Symplectic Geom. 4(3), 317343 (2006)

7. Enrietti, N., Fino, A., Vezzoni, L.: Tamed symplectic forms and strong Kähler with torsion metrics. J. Symplectic Geom. 10(2), 203-223 (2012)

8. Fino, A., Kasuya, H., Vezzoni, L.: SKT and tamed symplectic structures on solvmanifolds. Tohoku Math. J. (2) 67(1), 19-37 (2015) 
9. Fino, A., Otal, A., Ugarte, L.: Six-dimensional solvmanifolds with holomorphically trivial canonical bundle, Int. Math. Res. Not. IMRN, no. 24, 13757-13799 (2015)

10. Fino, A., Paradiso, F.: Generalized Kähler Almost Abelian Lie Groups, 08 (2020). arXiv:2008.00458 [math.DG]

11. Fino, A., Parton, M., Salamon, S.: Families of strong KT structures in six dimensions. Comment. Math. Helv. 79(2), 317-340 (2004)

12. Freibert, M., Swann, A.: The shear construction. Geom. Dedicata 198, 71-101 (2019)

13. Fino, A., Vezzoni, L.: A correction to "Tamed symplectic forms and strong Kähler with torsion metrics.". J. Symplectic Geom. 17(4), 1079-1081 (2019)

14. Gauduchon, P.: La 1-forme de torsion d'une variété hermitienne compacte. Math. Ann. 267(4), 495-518 (1984)

15. Gates Jr., S.J., Hull, C.M., Roček, M.: Twisted multiplets and new supersymmetric nonlinear $\sigma$-models. Nuclear Phys. B 248(1), 157-186 (1984)

16. Gong, M.-P.: Classification of nilpotent Lie algebras of dimension 7 (over algebraically closed fields and R), Ph.D. thesis, University of Waterloo (Canada), p. 165 (1998)

17. Howe, P.S., Papadopoulos, G.: Further remarks on the geometry of two-dimensional nonlinear $\sigma$ models. Classical Quantum Gravity 5(12), 1647-1661 (1988)

18. Madsen, T.B., Swann, A.: Invariant strong KT geometry on four-dimensional solvable Lie groups. J. Lie Theory 21(1), 55-70 (2011)

19. Mubarakzjanov, G.M.: Classification of real structures of Lie algebras of fifth order. Izv. Vyš̌. Učebn. Zaved. Matematika 1963(3 (34)), 99-106 (1963)

20. Mubarakzjanov, G.M.: Classification of solvable Lie algebras of sixth order with a non-nilpotent basis element. Izv. Vysš. Učebn. Zaved. Matematika 1963(4 (35)), 104-116 (1963)

21. Strominger, A.: Superstrings with torsion. Nuclear Phys. B 274(2), 253-284 (1986)

22. Swann, A.: Twisting Hermitian and hypercomplex geometries. Duke Math. J. 155(2), 403-431 (2010)

23. Turkowski, P.: Solvable Lie algebras of dimension six. J. Math. Phys. 31(6), 1344-1350 (1990)

Publisher's Note Springer Nature remains neutral with regard to jurisdictional claims in published maps and institutional affiliations. 